\title{
THE FESHBACH-KERMAN-KOONIN MULTISTEP DIRECT REACTION THEORY
}

\author{
R. BONETTI ${ }^{a}$, A.J KONING , J.M. AKKERMANS ${ }^{\text {c }}$, P.E. HODGSON ${ }^{d}$ \\ ${ }^{a}$ Istituto di Fisica Generale Applicata, Universita degli Studi di Milano, Italy \\ ${ }^{b}$ ECN, Petten, The Netherlands \\ ${ }^{c}$ University of Twente P.O. Box 217, 7500 AE Enschede, The Netherlands \\ d Department of Physics, University of Oxford, Keble Road, OXI 3RH Oxford, UK
}

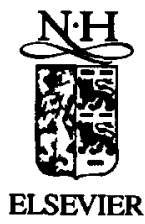

AMSTERDAM - LAUSANNE - NEW YORK - OXFORD - SHANNON - TOKYO 


\title{
The Feshbach-Kerman-Koonin multistep direct reaction theory
}

\author{
R. Bonetti ${ }^{\mathrm{a}}$, A.J Koning ${ }^{\mathrm{b}}$, J.M. Akkermans ${ }^{\text {c }}$, P.E. Hodgson ${ }^{\mathrm{d}}$ \\ "Istituto di Fisica Generale Applicata, Universita degli Studi di Milano, Italy \\ ${ }^{\mathrm{b}}$ ECN, Petten, The Netherlands \\ 'University of Twente P.O. Box 217,7500 AE Enschede, The Netherlands \\ ¿Department of Physics, University of Oxford, Keble Road, OXI 3RH Oxford. UK
}

Received October 1993; editor: G.E. Brown

\section{Contents}

1. Introduction

2. The multistep direct theory

2.1. The FKK multistep direct formula

2.2. The cross-section distribution

2.3. Non-normal DWBA matrix elements and energy averaging

2.4. Statistics

2.5. The one-step cross-section

2.6. The two-step cross-section

2.7. Discussion
3. Analyses of experimental data

3.1. The general method 30

3.2. Nucleon reactions below $30 \mathrm{MeV} \quad 31$

3.3. Reactions above $30 \mathrm{MeV} \quad 39$

3.4. A macroscopic computational model 46

3.5. Transitions from the $P$ - to the $Q$-chain $\quad 48$

3.6. Alpha-particle reactions 5.3

4. Conclusion 53

Appendix by M.B. Chadwick 54

References

\section{Abstract}

The multistep direct reaction theory of Feshbach, Kerman and Koonin (1980) is described in detail and compared with other theories. The results of several analyses of experimental cross-sections are described, and the validity and usefulness of the theory assessed. 


\section{Introduction}

In recent years evidence has accumulated for the emission of particles from nuclear reactions after the first interaction but before the attainment of statistical equilibrium. Experimentally, these 'pre-equilibrium' reactions are characterised by pronounced high-energy tails in the energy distribution of emitted particles, and by smooth, forward-peaked angular distributions. Griffin (1966) suggested that these reactions take place in a series of stages corresponding to the interaction of the projectile with nucleons in the nucleus, and that each of these interactions can lead to the excitation of a particle-hole pair. He formulated a simple exciton model of emission from each of these stages, and obtained good agreement with the energy distribution of the emitted particles. Subsequently the exciton model was extensively developed, and many reviews have been published (Blann, 1975; Gruppelaar et al., 1986; Gadioli and Hodgson, 1992).

A fully quantum mechanical theory of pre-equilibrium reactions was formulated by Feshbach (1973, 1977), Feshbach, Kerman and Koonin (FKK) (1980). This theory considers a chain of nuclear interactions initiated by the incident particle, and identifies two types of processes, the multistep direct and the multistep compound. In a statistical multistep direct emission (MSD) reaction at least one particle is in a continuum energy state, whereas in the statistical multistep compound emission (MSC) all the excited nucleons remain bound. At low energies multistep reactions take place mainly by the MSC process, and as the energy increases the MSD process becomes increasingly important and eventually dominates.

The multistep direct reaction theory is based on the physical picture of a fast incident particle in the continuum that creates new particle-hole. pairs on its way through the nucleus. This leading particle usually leaves the nucleus after a few interactions (steps) which implies that upon emission it retains some memory of the incident energy and direction. For the quantum-mechanical formulation of this process it is necessary to distinguish between intermediate states in which all particles are bound and those in which at least one particle is in the continuum. Formally, these states can be described by the projection operators $P$ and $Q$ acting on the total wavefunction $\Psi$, with $P+Q=1$. This divides the multistep process into two physically different types of reaction: the multistep direct reactions can be described by the propagation through the states $P \Psi$ and the multistep compound reactions by propagation through the states $Q \Psi$. The division into these two reaction types is shown in Fig. 1, where the various stages of increasing complexity are depicted. Decay from the $Q$-chain can take place via neighbouring $P$-states; propagation through the $Q$-chain eventually leads to a fully equilibrated excited compound nucleus which then decays statistically in accord with the Hauser-Feshbach compound reaction theory. The relative fluxes passing down the $P$ and the $Q$-chain depend strongly on the incident energy. At low energies the $Q$-chain is important, giving symmetric compound angular distributions. This multistep compound emission has been reviewed by Bonetti et al. (1991). As the energy increases the $P$-chain interactions become increasingly important until finally they dominate the reaction process giving forward-peaked multistep angular distributions, and high-energy tails in the emission spectra.

Several different theories have been proposed to account for MSD reactions. The FKK theory has become the one most widely applied to the analysis of experimental data. Currently, a variety of computer programs is available that carry out calculations according to the FKK MSD formalism.

The first of these studies were made by Avaldi et al. (1980) and by Bonetti et al. (1981), who calculated the cross-sections of several $(p, n)$ reactions from 25 to $45 \mathrm{MeV}$. On the whole the theory 


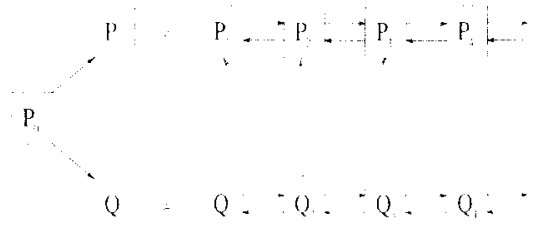

Fig. 1. Partition of Hilbert space into subspaces of increasing complexity indicated by the subscript. The $P$-chain refers to MSD reactions, and the $Q$-chain to MSC reactions.

gave a good overall fit to the experimental data, and showed the contributions of each step in the chain of interactions to the measured cross-section. At these energies the leading particle is in the continuum and the theory gives correctly the absolute values of the cross-sections and their variations with energy and angle for ${ }^{48} \mathrm{Ca},{ }^{90} \mathrm{Zr},{ }^{120} \mathrm{Sn}$ and ${ }^{208} \mathrm{~Pb}$. Subsequently, the calculations were extended to include the analyzing powers (Bonetti et al., 1982), and qualitative agreement was obtained with the experimental data of Sakai et al. (1980) for ${ }^{58} \mathrm{Ni}\left(\mathrm{p}, \mathrm{p}^{\prime}\right)$ at $65 \mathrm{MeV}$.

The quality of the fits to the data obtained in these MSD calculations is notably poorer for lower incident energies, and this was attributed to MSC emission. The theory was accordingly extended to enable the contribution of such processes to be evaluated, and calculations by Bonetti et al. $(1980,1982,1983,1984)$ showed that it is able to give the cross-sections at lower energies. Taken together, the MSC and MSD theories give a good overall account of the pre-equilibrium crosssections and analyzing powers in the energy range from 10 to $50 \mathrm{MeV}$ with consistent values of the two-body residual interaction (Bonetti and Colombo, 1983).

In the following years the FKK multistep direct formalism was used by many authors (Holler et al., 1985; Trabandt et al., 1988, 1989; Marcinkowski et al., 1989; Scobel et al., 1990; Cowley et al.. 1991; Richter et al., 1992; Stamer et al., 1993; Koning and Akkermans, 1993a) to analyse a wide range of $(p, n),\left(n, n^{\prime}\right)$ and $\left(p, p^{\prime}\right)$ reactions. On the whole, good overall agreement is found between the calculation and the experimental data, with consistent values of the parameters. This leads to the conclusion that the FKK multistep direct formalism is now established as a valid and reliable way of analyzing experimental reaction cross-sections for nucleons from 20 to up to at least $250 \mathrm{MeV}$

There have, however, been several other theories of the MSD reaction process based on postulates quite different from those used in the FKK theory. One of these has been developed by Tamura et al. (1977a, b, 1981, 1982) and by Lenske et al. (1983) and Udagawa et al. (1979, 1980. 1982,1983 ) as an extension into the continuum of the direct reaction formalism which they have been applying for many years to analyse reactions to discrete final states. This theory (TUL) was successful in fitting the experimental data on proton inelastic scattering by ${ }^{27} \mathrm{Al}$ and ${ }^{209} \mathrm{Bi}$ at $62 \mathrm{MeV}$, although its complexity makes it difficult to extend to processes with many steps. Tsai and Bertsch (1978) pointed out that the earlier version of this theory failed to give the absolute values of the cross-sections, but Tamura and Udagawa (1978) showed that this can be achieved by taking into account the effects of ground state correlations and the transfer of large angular momenta. Subsequently the TUL theory was extended to $(\mathrm{p}, \alpha)$ reactions using the triton pickup model and also to heavy ion reactions. 
Yet another quantum-mechanical theory of MSD reactions has been developed by Nishioka, Weidenmüller and Yoshida (NWY) (1988). However, this theory has not been extensively compared with experimental data (Koning and Akkermans, 1993). Finally there is the semi-classical exciton model which is still widely used in practice. Although it is a simple model, its statistical assumptions are related to the ones made in the quantum-mechanical theories (Akkermans and Koning, 1990).

There has been considerable discussion of the scope and validity of these theories. One important issue has become known as the $\chi-\hat{\chi}$ problem. It was pointed out by Kawai and independently by Udagawa et al. (1983) that the derivation of the FKK theory by Feshbach et al. (1980) gives expressions for the cross-sections in terms of non-DWBA matrix elements of the form $\left\langle\hat{\chi}^{(+)}|V| \chi^{(+)}\right\rangle$, whereas the FKK paper itself gives formulae containing standard DWBA matrix elements of the form $\left\langle\chi^{(-)}|V| \chi^{(+)}\right\rangle$. The latter result has, moreover, been used in all computational studies based on the FKK formalism. Furthermore, the use of non-DWBA matrix elements leads to poor fits to the experimental data.

This problem was studied by Feshbach (1985a, b, 1986a, b, 1992) who realised that the energyaveraging had been incorrectly carried out by FKK, and that when it is done correctly it has the effect of transforming the non-DWBA matrix elements into DWBA matrix elements. However, because of the intricate details of the mathematics involved, it is still too early to say that the $\chi-\hat{\chi}$ problem has been satisfactorily settled, and it is extensively discussed in the present review.

A second important issue is the relationship between various different MSD theories, and this has been extensively debated. The problem was clarified by Koning and Akkermans $(1991,1993)$ who derived in a clear and concise way, using certain statistical postulates, the theory of Feshbach, Kerman and Koonin, that of Tamura Udagawa and Lenske and that of Nishioka, Weidenmüller and Yoshida. They distinguished between two different statistical postulates, one being the random configuration mixing of the residual states and another the randomness of the DWBA matrix elements. Koning and Akkermans then showed that the TUL and NWY theories result from using only the first postulate, whereas the FKK theory also requires the second postulate. Therefore, since they are based on quite different statistical assumptions, it is not justified to consider the FKK theory as a simplification of the TUL theory as was done by Udagawa et al. (1983). This is briefly reviewed in the present paper.

This review is devoted to the FKK multistep direct theory. In Section 2 the formalism of the theory is described and in Section 3 it is used to analyse a range of experimental data. Section 4 gives some conclusions.

The FKK multistep compound theory has already been summarised by Bonetti et al. (1991) and we take this opportunity to correct and discuss some formulae in an Appendix by Dr. M.B. Chadwick.

\section{The multistep direct theory}

\subsection{The FKK multistep direct formula}

Using the separation into a $P$ - and a $Q$-chain and by combining direct reaction concepts with a statistical description of the nuclear levels, Feshbach et al. (1980) arrived at an appealing result for multistep direct emission in the continuum. The FKK cross-section can be written as an incoherent 
sum of a one-step term and multistep terms. Furthermore, each multistep term can be written as a convolution of one-step cross sections. The original formula of Feshbach et al. (1980) reads as follows (using a notation which is consistent with the rest of this review):

$$
\frac{\mathrm{d}^{2} \sigma\left(\boldsymbol{k}, \boldsymbol{k}_{0}\right)}{\mathrm{d} \Omega \mathrm{d} U}=\sum_{n=1}^{\infty} \frac{\mathrm{d}^{2} \sigma^{(n)}\left(\boldsymbol{k}, \boldsymbol{k}_{0}\right)}{\mathrm{d} \Omega \mathrm{d} U} .
$$

where the one-step cross-section is given by

$$
\frac{\mathrm{d}^{2} \sigma^{(\boldsymbol{1})}\left(\boldsymbol{k}, \boldsymbol{k}_{0}\right)}{\mathrm{d} \Omega_{1} \mathrm{~d} U_{1}}=\frac{2 \pi m}{\hbar^{2} k_{0}} \rho(k) \rho_{1 p 1 h}\left(U_{1}\right)\left|M_{1,0}\left(\boldsymbol{k}_{1}, \boldsymbol{k}_{0}\right)\right|^{2}
$$

and the $n$-step cross-section is given by

$$
\begin{aligned}
\frac{\mathrm{d}^{2} \sigma^{(n)}\left(\boldsymbol{k}, \boldsymbol{k}_{0}\right)}{\mathrm{d} \Omega \mathrm{d} U}= & \int \frac{\mathrm{d} \boldsymbol{k}_{1}}{(2 \pi)^{3}} \cdots \frac{\mathrm{d} \boldsymbol{k}_{n}}{(2 \pi)^{3}} \frac{\mathrm{d}^{2} w_{i, n}\left(\boldsymbol{k}, \boldsymbol{k}_{n}\right)}{\mathrm{d} \Omega \mathrm{d} U} \\
& \times \frac{\mathrm{d}^{2} w_{n, n-1}\left(\boldsymbol{k}_{n}, \boldsymbol{k}_{n-1}\right)}{\mathrm{d} \Omega_{n} \mathrm{~d} U_{n}} \cdots \frac{\mathrm{d}^{2} w_{2,1}\left(\boldsymbol{k}_{2}, \boldsymbol{k}_{1}\right)}{\mathrm{d} \Omega_{2} \mathrm{~d} U_{2}} \frac{\mathrm{d}^{2} \sigma^{(1)}\left(\boldsymbol{k}_{1}, \boldsymbol{k}_{0}\right)}{\mathrm{d} \Omega_{1} \mathrm{~d} U_{1}} .
\end{aligned}
$$

Here, $w$ is the transition probability from the $(n-1)$ th to the $n$th stage, whereby the particle momentum changes from $\boldsymbol{k}_{n-1}$ to $\boldsymbol{k}_{n}$ :

$$
\frac{\mathrm{d}^{2} w_{n, n-1}\left(\boldsymbol{k}_{n}, \boldsymbol{k}_{n-1}\right)}{\mathrm{d} \Omega_{n} \mathrm{~d} U_{n}}=2 \pi^{2} \rho\left(k_{n}\right) \rho_{1 p 1 h}\left(U_{n}\right)\left|M_{n, n-1}\left(\boldsymbol{k}_{n}, \boldsymbol{k}_{n-1}\right)\right|^{2} .
$$

where $\rho_{1 p 1 h}\left(U_{n}\right)$ is the level density of the residual nucleus at the (stage-dependent) excitation energy $U_{n}, M_{n, n-1}$ is a DWBA matrix element for the transition from the $(n-1)$ th stage to the $n$th stage and the state density of the continuum leading particle is

$$
\rho\left(k_{n}\right)=m k_{n} /(2 \pi)^{3} h^{2} .
$$

As we will show now, the FKK equations (2.1)-(2.4) can be written in an equivalent and more compact form (Koning and Akkermans, 1993) by expressing them in terms of energy-solid angle variables instead of the momentum $\boldsymbol{k}$. This form is more suitable for numerical calculations and will also be the end result of the derivation that we present hereafter.

Eq. (2.2) illustrates that the one-step cross-section for the continuum can be written as the product of an averaged matrix element and the level density. Using the expression for the DWBA cross-section

$$
\frac{\mathrm{d} \sigma\left(\boldsymbol{k}, \boldsymbol{k}_{0}\right)^{\mathrm{DWBA}}}{\mathrm{d} \Omega}=\frac{m^{2}}{\left(2 \pi \hbar^{2}\right)^{2}} \frac{k}{k_{0}}\left|M\left(\boldsymbol{k}, \boldsymbol{k}_{0}\right)\right|^{2},
$$

and Eq. (2.5), we see that upon decomposition into different transferred angular momenta $J$. Eq. (2.2) becomes

$$
\frac{\mathrm{d}^{2} \sigma^{(1)}\left(\boldsymbol{k}, \boldsymbol{k}_{0}\right)}{\mathrm{d} \Omega \mathrm{d} U}=\sum_{J} \rho_{1 p 1 \boldsymbol{h}, J}(U)\left[\frac{\mathrm{d} \sigma\left(\boldsymbol{k} \leftarrow \boldsymbol{k}_{0}\right)}{\mathrm{d} \Omega}\right]_{J}^{\mathrm{DWBA}},
$$

where $\rho_{1 p 1 h, J}$ is the level density per angular momentum and the quantity between brackets is an averaged DWBA cross-section per angular momentum. 
In order to see how the multistep cross-section can be rewritten, we consider the two-step term of Eq. (2.3):

$$
\frac{\mathrm{d}^{2} \sigma^{(2)}\left(\boldsymbol{k}, \boldsymbol{k}_{0}\right)}{\mathrm{d} \Omega \mathrm{d} U}=\int \frac{\mathrm{d} \boldsymbol{k}_{1}}{(2 \pi)^{3}} \frac{\mathrm{d}^{2} w_{2,1}\left(\boldsymbol{k}, \boldsymbol{k}_{1}\right)}{\mathrm{d} \Omega \mathrm{d} U} \frac{\mathrm{d}^{2} \sigma^{(1)}\left(\boldsymbol{k}_{1}, \boldsymbol{k}_{0}\right)}{\mathrm{d} \Omega_{1} \mathrm{~d} U_{1}} .
$$

Inserting Eqs. (2.2), (2.4) and (2.5) in this equation yields

$$
\begin{aligned}
\frac{\mathrm{d}^{2} \sigma^{(2)}\left(\boldsymbol{k}, \boldsymbol{k}_{0}\right)}{\mathrm{d} \Omega \mathrm{d} U}= & \frac{m^{3} k}{2(2 \pi)^{6} \hbar^{6} k_{0}} \int \mathrm{d} \boldsymbol{k}_{1} k_{1} \rho_{1 p 1 h}(U)\left|M_{2,1}\left(\boldsymbol{k}, \boldsymbol{k}_{1}\right)\right|^{2} \rho_{1 p 1 h}\left(U_{1}\right) \\
& \times\left|M_{1,0}\left(\boldsymbol{k}_{1}, \boldsymbol{k}_{0}\right)\right|^{2} .
\end{aligned}
$$

Decomposition into $J$ and using the expression for the DWBA cross-section (2.6) twice gives

$$
\begin{aligned}
\frac{\mathrm{d}^{2} \sigma^{(2)}\left(\boldsymbol{k}, \boldsymbol{k}_{0}\right)}{\mathrm{d} \Omega \mathrm{d} U}= & \frac{\hbar^{2}}{2(2 \pi)^{2} m} \sum_{J_{1}, J_{2}} \int \mathrm{d} \boldsymbol{k}_{1} k_{1} \rho_{1 p 1 h, J_{2}}(U)\left[\frac{\mathrm{d} \sigma\left(\boldsymbol{k} \leftarrow \boldsymbol{k}_{1}\right)}{\mathrm{d} \Omega}\right]_{J_{2}}^{\mathrm{DWBA}} \\
& \times \rho_{1 p 1 h, J_{1}}\left(U_{1}\right)\left[\frac{\mathrm{d} \sigma\left(\boldsymbol{k}_{1} \leftarrow \boldsymbol{k}_{0}\right)}{\mathrm{d} \Omega}\right]_{J_{1}}^{\mathrm{DWBA}}
\end{aligned}
$$

Next, we choose a representation in terms of the intermediate leading particle energy $E_{k_{1}}$ and intermediate solid angle $\Omega_{1}$, by means of the relations

$$
E_{k_{1}}=\frac{\hbar^{2} k_{1}^{2}}{2 m}, \quad \int \mathrm{d} \boldsymbol{k}_{1}=\int \mathrm{d} \Omega_{1} \int \mathrm{d} E_{k_{1}} \frac{m\left(2 m E_{k_{1}}\right)^{1 / 2}}{\hbar^{3}} .
$$

Then the two-step cross-section (2.10) can be written as follows:

$$
\begin{aligned}
\frac{\mathrm{d}^{2} \sigma^{(2)}\left(E_{k}, \Omega \leftarrow E_{k_{0}}, \Omega_{0}\right)}{\mathrm{d} \Omega \mathrm{d} E_{k}}= & \frac{m}{4 \pi^{2} \hbar^{2}} \int \mathrm{d} \Omega_{1} \int \mathrm{d} E_{k_{1}} E_{k_{1}} \frac{\mathrm{d}^{2} \sigma^{(1)}\left(E_{k}, \Omega \leftarrow E_{k_{1}}, \Omega_{1}\right)}{\mathrm{d} \Omega \mathrm{d} E_{k}} \\
& \times \frac{\mathrm{d}^{2} \sigma^{(1)}\left(E_{k_{1}}, \Omega_{1} \leftarrow E_{k_{0}}, \Omega_{0}\right)}{\mathrm{d} \Omega_{1} \mathrm{~d} E_{k_{1}}} .
\end{aligned}
$$

For the higher steps, an analogous procedure is followed. The $n$-step cross-section is

$$
\begin{aligned}
\frac{\mathrm{d}^{2} \sigma^{(n)}\left(E_{k}, \Omega \leftarrow E_{k_{0}}, \Omega_{0}\right)}{\mathrm{d} \Omega \mathrm{d} E_{k}}= & \left(\frac{m}{4 \pi^{2} \hbar^{2}}\right)^{n-1} \int \mathrm{d} \Omega_{n-1} \int \mathrm{d} E_{k_{n-1}} E_{k_{n-1}} \cdots \int \mathrm{d} \Omega_{1} \int \mathrm{d} E_{k_{1}} \\
& \times E_{k_{1}} \frac{\mathrm{d}^{2} \sigma^{(1)}\left(E_{k}, \Omega \leftarrow E_{k_{n-1}}, \Omega_{n-1}\right)}{\mathrm{d} \Omega \mathrm{d} E_{k}} \ldots \frac{\mathrm{d}^{2} \sigma^{(1)}\left(E_{k_{1}}, \Omega_{1} \leftarrow E_{k_{0}}, \Omega_{0}\right)}{\mathrm{d} \Omega_{1} \mathrm{~d} E_{k_{1}}} .
\end{aligned}
$$

Collecting these results, we have for the total FKK cross-section for (multistep) direct processes

$$
\frac{\mathrm{d}^{2} \sigma\left(E_{k}, \Omega \leftarrow E_{k_{0}}, \Omega_{0}\right)}{\mathrm{d} \Omega \mathrm{d} E_{k}}=\sum_{n=1}^{\infty} \frac{\mathrm{d}^{2} \sigma^{(n)}\left(E_{k}, \Omega \leftarrow E_{k_{0}}, \Omega_{0}\right)}{\mathrm{d} \Omega \mathrm{d} E_{k}} .
$$


The first step cross-section reads

$$
\frac{\mathrm{d}^{2} \sigma^{(1)}\left(E_{k}, \Omega \leftarrow E_{k_{0}}, \Omega_{0}\right)}{\mathrm{d} \Omega \mathrm{d} E_{k}}=\sum_{I} \rho_{1 p 1 h . J}\left(E_{k_{0}}-E_{k}\right)\left[\frac{\mathrm{d} \sigma\left(E_{k}, \Omega \leftarrow E_{k_{0}}, \Omega_{0}\right)}{\mathrm{d} \Omega}\right]_{J}^{\text {DW }}
$$

and the $n$-step $(n>1)$ cross-section can be written in the following recursive way:

$$
\begin{aligned}
\frac{\mathrm{d}^{2} \sigma^{(n)}\left(E_{k}, \Omega \leftarrow E_{k_{0}}, \Omega_{0}\right)}{\mathrm{d} \Omega \mathrm{d} E_{k}}= & \frac{m}{4 \pi^{2} \hbar^{2}} \int \mathrm{d} \Omega_{n-1} \int \mathrm{d} E_{k_{n+1}} E_{k_{n-1}} \\
& \times \frac{\mathrm{d}^{2} \sigma^{(1)}\left(E_{k}, \Omega \leftarrow E_{k_{n}+1}, \Omega_{n-1}\right)}{\mathrm{d} \Omega \mathrm{d} E_{k}} \frac{\mathrm{d}^{2} \sigma^{(n-1)}\left(E_{k_{n},}, \Omega_{n-1} \leftarrow E_{k_{0}}, \Omega_{0}\right)}{\mathrm{d} \Omega_{n+1} \mathrm{~d} E_{k_{n}}} .
\end{aligned}
$$

The most conspicuous feature of the convolution structure of the FKK model is that the $n$-step cross-section can be expressed in terms of the result of the previous stage and is therefore easy to calculate. Basically, only first order DWBA cross-sections and $1 p 1 h$ level densities are required as input for the calculation of the multistep cross section.

The remainder of this chapter is devoted to the derivation of the FKK equations (2.14)-(2.16).

\subsection{The cross-section distribution}

In this section, an expression will be derived for the multistep direct cross-section distribution that is representative of the reaction process before any statistical hypothesis has been introduced. For simplicity, we confine ourselves to nucleon-induced inelastic scattering. We follow the notation of Koning and Akkermans (1991).

Consider a system of a nucleon incident on a target nucleus consisting of $(A-1)$ nucleons. The Hamiltonian for this system is

$$
H=H_{0}+H_{1}+K(A)+U_{\mathrm{opt}}(A)+V .
$$

Here, the shell-model Hamiltonian $H_{0}$ of the residual nucleus is

$$
H_{0}=\sum_{i=1}^{A-1}[K(i)+U(i)] .
$$

where $K$ is the kinetic energy and $U$ is the shell-model potential. The residual interaction of the residual nucleus $H_{1}$ is

$$
H_{1}=\sum_{i<j}^{A-1} v(i, j)-\sum_{i=1}^{A-1} U(i),
$$

where $v$ is the mutual interaction between the nucleons. The relative motion of the leading particle (labelled by $A$ ) in the mean field of the residual nucleus is described by the leading-particle Hamiltonian $K(A)+U_{\mathrm{opt}}(A)$, where $U_{\mathrm{opt}}$ is the optical potential. Finally, the perturbative term

$$
V=\sum_{i=1}^{A-1} v(i, A)-U_{\mathrm{opt}}(A)
$$


represents the residual interaction of the leading particle, which is assumed to be a two-body operator.

The states of the target nucleus $|n\rangle$ (with eigenenergies $E_{n}$ ) are eigenstates of $H_{0}+H_{1}$ :

$$
\left(H_{0}+H_{1}\right)|n\rangle=E_{n}|n\rangle \text {. }
$$

The shell-model Hamiltonian $H_{0}$ generates a complete set of particle-hole eigenstates $|m \mu\rangle$

$$
H_{0}|m \mu\rangle=E_{m \mu}|m \mu\rangle,
$$

where $m$ determines the exciton class (mpmh-states), $\mu$ is an index for the particular particle-hole configuration within a class and $E_{m \mu}$ is the energy of the particle-hole state.

The presence of the residual interaction $H_{1}$ causes configuration mixing and this is represented by the expansion of $|n\rangle$ as a linear combination of particle-hole states

$$
|n\rangle=\sum_{m \mu} a_{m \mu}^{n}|m \mu\rangle \text {. }
$$

Thus, each particle-hole state $|m \mu\rangle$ is distributed over the real states $|n\rangle$ and the strength of each contribution is determined by the distribution amplitude $a_{m \mu}^{n}$.

The dynamics of the leading particle is described by distorted waves $\chi$. Since the optical potential is complex, these distorted waves $\chi$ do not form a complete set of states by themselves and have to be supplemented by the bi-orthogonally conjugated functions $\hat{\chi}$ (Satchler, 1983). We have the eigenvalue equations

$$
\begin{aligned}
& {\left[E_{k}-K-U_{\mathrm{opt}}\right]\left|\chi^{(+)}(\boldsymbol{k})\right\rangle=0,} \\
& {\left[E_{k}-K-U_{\mathrm{opt}}^{\dagger}\right]\left|\hat{\chi}^{(+)}(\boldsymbol{k})\right\rangle=0,}
\end{aligned}
$$

where $E_{k}$ is the relative kinetic energy and the $(+)$ designates the outgoing solution. These distorted waves satisfy the orthonormality and completeness relations

$$
\left\langle\chi^{(+)}(\boldsymbol{k}) \mid \hat{\chi}^{(+)}\left(\boldsymbol{k}^{\prime}\right)\right\rangle=(2 \pi)^{3} \delta\left(\boldsymbol{k}-\boldsymbol{k}^{\prime}\right), \quad \int \frac{\mathrm{d} \boldsymbol{k}}{(2 \pi)^{3}}\left|\chi^{(+)}(\boldsymbol{k})\right\rangle\left\langle\hat{\chi}^{(+)}(\boldsymbol{k})\right|=1 .
$$

We will discuss these distorted waves more extensively in Section 2.3, where we discuss the operation (i.e. energy averaging) that is necessary to bring the FKK cross-section into a DWBA form.

It is now possible to construct anti-symmetrized direct products of the residual nuclear states and the distorted waves and use them as a complete set of intermediate states in the multistep process. In this way, the transition amplitude for going from the initial state $|0\rangle\left|\chi^{(+)}\left(\boldsymbol{k}_{\mathrm{o}}\right)\right\rangle$ to the final state $|f\rangle\left|\chi^{(-)}(k)\right\rangle$ can be given by the Born series

$$
\begin{aligned}
T_{f-0} & =\left\langle\chi^{(-)}(\boldsymbol{k})|\langle f|V+V G V+V G V G V+\cdots| 0\rangle| \chi^{(+)}\left(\boldsymbol{k}_{0}\right)\right\rangle \\
& =\sum_{i=1}^{\infty} T_{f \leftarrow 0}^{(i)},
\end{aligned}
$$

where

$$
G=\sum_{n} \int \frac{\mathrm{d} \boldsymbol{k}_{1}}{(2 \pi)^{3}} \frac{|n\rangle\left|\chi^{(+)}\left(\boldsymbol{k}_{1}\right)\right\rangle\left\langle\hat{\chi}^{(+)}\left(\boldsymbol{k}_{1}\right)\right|\langle n|}{E-E_{n}-E_{k_{1}}+\mathrm{i} \varepsilon} .
$$


In particular, the two-step transition amplitude reads

$$
\begin{aligned}
& T_{f \leftarrow 0}^{(2)}= \sum_{n} \int \frac{\mathrm{d} \boldsymbol{k}_{1}}{(2 \pi)^{3}}\left\langle\chi^{(-)}(\boldsymbol{k})|\langle f|V| n\rangle| \chi^{(+)}\left(\boldsymbol{k}_{1}\right)\right\rangle \frac{1}{E-E_{n}-E_{k_{1}}+\mathrm{i} \varepsilon} \\
&=\quad \times\left\langle\hat{\chi}^{(+)}\left(\boldsymbol{k}_{1}\right)|\langle n|V| 0\rangle| \chi^{(+)}\left(\boldsymbol{k}_{0}\right)\right\rangle .
\end{aligned}
$$

Although we here employ the distorted wave Born approximation, we note that the derivation can be generalised so as to deal with the case of strong interactions, as shown by Feshbach et al. (1980).

Multistep direct reactions are defined as multistage processes that propagate through the open intermediate configurations only. Therefore, we define the multistep direct Born series by Eq. (2.26) with the integral over the absolute value of $\boldsymbol{k}_{1}$ running from $k_{\mathrm{B}}$, the absolute value of the momentum that corresponds to the binding energy, to $\infty$. This defines the $P$-space. If the energy of the leading particle lies below the binding energy, it is no longer distinguishable and the present description will lose its validity. For these bound configurations one will have to adopt the multistep compound reaction formalism.

Now that we have established the perturbation expansion of the transition amplitude, we can define the cross-section distribution

$$
\frac{\mathrm{d}^{2} \sigma^{\mathrm{dis}}}{\mathrm{d} \Omega \mathrm{d} E_{k}}=\frac{m^{2}}{\left(2 \pi \hbar^{2}\right)^{2}} \frac{k}{k_{0}} \sum_{f}\left|T_{f \leftarrow 0}\right|^{2} \delta\left(E_{f}-U\right),
$$

where $U$ is the residual excitation energy. This formula reduces to the cross-section formula for discrete transitions when it is integrated over a small energy region around one particular state. Here, however, we have introduced it for the purpose of averaging over many continuum states. The cross-section distribution may be visualized as a kind of book-keeping detector that moves along the level scheme of the residual nucleus, recording each time an eigenstate is encountered.

Inserting Eq. (2.26) into Eq. (2.29) yields

$$
\begin{aligned}
\frac{\mathrm{d}^{2} \sigma^{\mathrm{dis}}}{\mathrm{d} \Omega \mathrm{d} E_{k}} & =\frac{m^{2}}{\left(2 \pi \hbar^{2}\right)^{2}} \frac{k}{k_{0}} \sum_{f}\left|\sum_{i} T_{f \leftarrow 0}^{(i)}\right|^{2} \delta\left(E_{f}-U\right) \\
& =\frac{m^{2}}{\left(2 \pi \hbar^{2}\right)^{2}} \frac{k}{k_{0}} \sum_{i} \sum_{f}\left|T_{f \leftarrow 0}^{(i)}\right|^{2} \delta\left(E_{f}-U\right)+\text { cross-terms } \\
& \equiv \frac{m^{2}}{\left(2 \pi \hbar^{2}\right)^{2}} \frac{k}{k_{0}} \sum_{i} \frac{\mathrm{d}^{2} \sigma^{(i)^{(i)}}}{\mathrm{d} \Omega \mathrm{d} E_{k}}+\text { cross-terms }
\end{aligned}
$$

which illustrates the decomposition into one-step $(i=1)$, multistep $(i>1)$ and interference terms.

We can now take the first term of Eq. (2.26) and use the linear combination (2.23) to obtain for the one-step cross-section distribution

$$
\begin{aligned}
\frac{\mathrm{d}^{2} \sigma^{(1)^{\mathrm{dik}}}}{\mathrm{d} \Omega \mathrm{d} E_{k}}= & \frac{m^{2}}{\left(2 \pi \hbar^{2}\right)^{2}} \frac{k}{k_{0}} \sum_{f}\left|T_{f}^{(1)}{ }_{0}\right|^{2} \delta\left(E_{f}-U\right) \\
= & \frac{m^{2}}{\left(2 \pi \hbar^{2}\right)^{2}} \frac{k}{k_{0}} \sum_{f} \sum_{\mu} \sum_{\mu^{\prime}} a_{1 \mu^{\prime}}^{f} a_{1 \mu}^{f}\left\langle\chi^{(+)}\left(\boldsymbol{k}_{0}\right)\left|\left\langle 0|V| 1 \mu^{\prime}\right\rangle\right| \chi^{(-)}(\boldsymbol{k})\right\rangle \\
& \times\left\langle\chi^{(-)}(\boldsymbol{k})|\langle 1 \mu|V| 0\rangle| \chi^{(+)}\left(\boldsymbol{k}_{0}\right)\right\rangle \delta\left(E_{f}-U\right) .
\end{aligned}
$$


Observe that the two-body nature of $V$ implies that only $1 \mathrm{p} 1 \mathrm{~h}$ states can be created in a one-step process. In other words, the more complex particle-hole terms of the linear combination (2.23) are not included in the one-step cross-section.

With Eq. (2.28), a similar expansion for the two-step cross-section distribution can be obtained

$$
\begin{aligned}
\frac{\mathrm{d}^{2} \sigma^{(2)^{\mathrm{dis}}}}{\mathrm{d} \Omega \mathrm{d} E_{k}}= & \frac{m^{2}}{\left(2 \pi \hbar^{2}\right)^{2}} \frac{k}{k_{0}} \sum_{f}\left|T_{f-0}^{(2)}\right|^{2} \delta\left(E_{f}-U\right) \\
= & \frac{m^{2}}{\left(2 \pi \hbar^{2}\right)^{2}} \frac{k}{k_{0}} \sum_{f} \sum_{n} \sum_{n^{\prime}} \sum_{\mu} \sum_{\mu^{\prime}} \int \frac{\mathrm{d} \boldsymbol{k}_{1}^{\prime}}{(2 \pi)^{3}} \int \frac{\mathrm{d} \boldsymbol{k}_{1}}{(2 \pi)^{3}}\left\langle\chi^{(+)}\left(\boldsymbol{k}_{0}\right)\left|\left\langle 0|V| 1 \mu^{\prime}\right\rangle\right| \hat{\chi}^{(+)}\left(\boldsymbol{k}_{1}^{\prime}\right)\right\rangle a_{1 \mu^{\prime}}^{n^{\prime}} \\
& \times \frac{1}{E-E_{n^{\prime}}-E_{k_{1}^{\prime}}-\mathrm{i} \varepsilon}\left\langle\chi^{(+)}\left(\boldsymbol{k}_{1}^{\prime}\right)\left|\left\langle\boldsymbol{n}^{\prime}|V| f\right\rangle\right| \chi^{(-)}(\boldsymbol{k})\right\rangle\left\langle\chi^{(-)}(\boldsymbol{k})|\langle f|V| n\rangle| \chi^{(+)}\left(\boldsymbol{k}_{1}\right)\right\rangle \\
& \times a_{1 \mu}^{n} \frac{1}{E-E_{n}-E_{\boldsymbol{k}_{1}}+\mathrm{i} \varepsilon}\left\langle\hat{\chi}^{(+)}\left(\boldsymbol{k}_{1}\right)|\langle 1 \mu|V| 0\rangle| \chi^{(+)}\left(\boldsymbol{k}_{0}\right)\right\rangle \delta\left(E_{f}-U\right) .
\end{aligned}
$$

In the following it will be shown how, by means of energy averaging and a statistical assumption, Eqs. (2.31) and (2.32) can be turned into the FKK one-step cross-section and two-step crosssection, respectively.

\subsection{Non-normal DWBA matrix elements and energy averaging}

At this point in the derivation, we come to an issue that has received much attention during the last decade. The philosophy behind the FKK model is clear: starting from the framework of distorted wave theory one comes to an expression for the cross section in the continuum [i.e. Eq. (2.32)], that still contains all interference terms and complicated multistep processes, with a deterministic coupling (with intermediate states and Green functions) of the initial state with the final state. The obvious strategy is to invoke (statistical) approximations so that the non-diagonal terms of Eq. (2.32) will cancel and that the (squared) two-step matrix elements can be decoupled into two (squared) one-step matrix elements. In this way, one would end up with a continuum two-step cross-section that is the product of two one-step cross-sections, as in Eq. (2.12).

However, there is one major obstacle to overcome before we can arrive at this conceptually appealing result. Decoupling the two-step terms of Eq. (2.32) into two one-step terms yields a normal DWBA matrix element

$$
\left\langle\chi^{(-)}(\boldsymbol{k})|\cdots| \chi^{(+)}\left(\boldsymbol{k}_{1}\right)\right\rangle
$$

and a non-normal DWBA matrix element

$$
\left\langle\hat{\chi}^{(+)}\left(\boldsymbol{k}_{1}\right)|\cdots| \chi^{(+)}\left(\boldsymbol{k}_{0}\right)\right\rangle,
$$

whereas the product of two one-step cross-sections, see Eq. (2.12), consists of two squared normal DWBA matrix elements only. The non-normal DWBA matrix element (2.34) differs from Eq. (2.33) in two respects. Firstly, the final distorted wave of Eq.(2.34) is an eigenfunction of the Hamiltonian with the complex conjugated optical potential (which is designated by the hat symbol). Secondly, it has a different boundary condition. This suggests that, apart from statistical assumptions, an 
additional operation is required to obtain the convolution-type cross section. Whether or not this is allowed has been subject to much debate in the literature. Feshbach (1985) has argued that an additional energy average should be performed before any statistical hypothesis can be applied. Then, upon this energy averaging, we obtain

$$
\left\langle\hat{\chi}^{(+)}\right\rangle_{E} \rightarrow \bar{\chi}^{(-)} \text {. }
$$

i.e. the outgoing distorted wave is converted into an energy-averaged incoming distorted wave and Eq. (2.34) is transformed into a normal DWBA matrix element, which solves the problem. This replacement only applies to the interaction region. As emphasized by Feshbach, this operation is, apart from being merely convenient because of the obtained result, essential because interference effects that normally cancel out in the single-channel case would give rise to anomalous behaviour of the continuum cross section if statistical assumptions are carried out before the extra energy averaging.

In this section, an exposition will be presented of the arguments that lead to Eq. (2.35).

\subsubsection{Distorted waves and S-matrix elements}

First, we look in more detail at the mathematics of the distorted waves that were already introduced in Eq. (2.24). The set of distorted waves obey the eigenvalue equations

$$
\begin{aligned}
& {\left[E_{k}-K-U_{\mathrm{opt}}\right] \hat{\chi}^{(+)}(\boldsymbol{k})=0,} \\
& {\left[E_{k}-K-U_{\mathrm{opt}}^{\dagger}\right] \hat{\chi}^{(+)}(\boldsymbol{k})=0,} \\
& {\left[E_{k}-K-U_{\mathrm{opt}}^{\dagger}\right] \chi^{(-)}(\boldsymbol{k})=0,} \\
& {\left[E_{k}-K-U_{\mathrm{opt}}\right] \hat{\chi}^{(-)}(\boldsymbol{k})=0,}
\end{aligned}
$$

and the orthonormality and completeness relations are

$$
\left\langle\chi^{( \pm)}(\boldsymbol{k}) \mid \hat{\chi}^{( \pm)}\left(\boldsymbol{k}^{\prime}\right)\right\rangle=(2 \pi)^{3} \delta\left(\boldsymbol{k}-\boldsymbol{k}^{\prime}\right), \int \frac{\mathrm{d} \boldsymbol{k}}{(2 \pi)^{3}}\left|\chi^{ \pm \pm}(\boldsymbol{k})\right\rangle\left\langle\hat{\chi}^{(+'}(\boldsymbol{k})\right|=1 .
$$

The $(+)$ designates the outgoing solution and the $(-)$ the incoming solution. For convenience, we have omitted the $\boldsymbol{r}$-dependence of the distorted waves. The complete solution of Eqs. (2.36) can be obtained by adding the homogeneous solution $\phi$ and by specifying the appropriate boundary conditions. Then, with the Lippmann-Schwinger technique, we obtain

$$
\begin{aligned}
& \chi^{(+)}(\boldsymbol{k})=\phi(\boldsymbol{k})+\frac{1}{E-K-U_{\mathrm{opt}}+\mathrm{i} \varepsilon} U_{\mathrm{opt}} \phi(\boldsymbol{k}) . \\
& \hat{\chi}^{(+)}(\boldsymbol{k})=\phi(\boldsymbol{k})+\frac{1}{E-K-U_{\mathrm{opt}}^{\dagger}+\mathrm{i} \varepsilon} U_{\mathrm{opt}}^{+} \phi(\boldsymbol{k}) \\
& \chi^{(-)}(\boldsymbol{k})=\phi(\boldsymbol{k})+\frac{1}{E-K-U_{\mathrm{opt}}^{\dagger}-\mathrm{i} \delta} U_{\mathrm{opt}}^{\dagger} \phi(\boldsymbol{k}), \\
& \hat{\chi}^{(-)}(\boldsymbol{k})=\phi(\boldsymbol{k})+\frac{1}{E-K-U_{\mathrm{opt}}-\mathrm{i} \varepsilon} U_{\mathrm{opt}} \phi(\boldsymbol{k}) .
\end{aligned}
$$


These distorted waves have asymptotically the form

$$
\begin{aligned}
& \lim _{r \rightarrow \infty} \chi^{(+)}(\boldsymbol{k}) \sim \mathrm{e}^{\mathrm{i} k \cdot r}+f(k) \frac{\mathrm{e}^{\mathrm{i} k r}}{r}, \\
& \lim _{r \rightarrow \infty} \hat{\chi}^{(+)}(\boldsymbol{k}) \sim \mathrm{e}^{\mathrm{j} k \cdot r}+\hat{f}(\boldsymbol{k}) \frac{\mathrm{e}^{\mathrm{i} k r}}{r}, \\
& \lim _{r \rightarrow \infty} \chi^{(-)}(\boldsymbol{k}) \sim \mathrm{e}^{\mathrm{i} k \cdot r}+f^{*}(-\boldsymbol{k}) \frac{\mathrm{e}^{-\mathrm{i} k r}}{r}, \\
& \lim _{r \rightarrow \infty} \hat{\chi}^{(-)}(\boldsymbol{k}) \sim \mathrm{e}^{\mathrm{i} k \cdot r}+\hat{f}^{*}(-\boldsymbol{k}) \frac{\mathrm{e}^{-\mathrm{i} k r}}{r},
\end{aligned}
$$

where $f$ is the scattering amplitude. Note that $\hat{\chi}^{(+)}$and $\chi^{(-)}$satisfy the same eigenvalue equation but obey different boundary conditions.

In order to write the transition amplitude (2.28) in terms of two DWBA matrix elements, we relate $\hat{\chi}^{+}$to $\chi^{-}$with the help of the completeness relation (2.37)

$$
\left\langle\hat{\chi}^{(+)}(\boldsymbol{k})\right|=\int \frac{\mathrm{d} \boldsymbol{k}^{\prime}}{(2 \pi)^{3}}\left\langle\hat{\chi}^{(+)}(\boldsymbol{k}) \mid \hat{\chi}^{(-)}\left(\boldsymbol{k}^{\prime}\right)\right\rangle\left\langle\chi^{(-)}\left(\boldsymbol{k}^{\prime}\right)\right| .
$$

Inserting this into Eq. (2.28) yields

$$
\begin{aligned}
T_{f \leftarrow 0}^{(2)}= & \sum_{n} \int \frac{\mathrm{d} \boldsymbol{k}_{1}}{(2 \pi)^{3}} \int \frac{\mathrm{d} \boldsymbol{k}_{1}^{\prime}}{(2 \pi)^{3}}\left\langle\chi^{(-)}(\boldsymbol{k})|\langle f|V| n\rangle| \chi^{(+)}\left(\boldsymbol{k}_{1}\right)\right\rangle \frac{1}{E-E_{n}-E_{k_{1}}+\mathrm{i} \varepsilon} \\
& \times\left\langle\hat{\chi}^{(+)}\left(\boldsymbol{k}_{1}\right) \mid \hat{\chi}^{(-)}\left(\boldsymbol{k}_{1}^{\prime}\right)\right\rangle\left\langle\chi^{(-)}\left(\boldsymbol{k}_{1}^{\prime}\right)|\langle n|V| 0\rangle| \chi^{(+)}\left(\boldsymbol{k}_{0}\right)\right\rangle .
\end{aligned}
$$

Similarly, the two-step cross-section distribution (2.32) becomes

$$
\begin{aligned}
\frac{\mathrm{d}^{2} \sigma^{(2)^{\mathrm{dik}}}}{\mathrm{d} \Omega \mathrm{d} E_{k}}= & \frac{m^{2}}{\left(2 \pi \hbar^{2}\right)^{2}} \frac{k}{k_{0}} \sum_{f}\left|T_{f-0}^{(2)}\right|^{2} \delta\left(E_{f}-U\right) \\
= & \frac{m^{2}}{\left(2 \pi \hbar^{2}\right)^{2}} \frac{k}{k_{0}} \sum_{f} \sum_{n} \sum_{n^{\prime}} \sum_{\mu} \sum_{\mu^{\prime}} \int \frac{\mathrm{d} \boldsymbol{k}_{1}^{\prime}}{(2 \pi)^{3}} \int \frac{\mathrm{d} \boldsymbol{k}_{1}}{(2 \pi)^{3}} \int \frac{\mathrm{d} \kappa_{1}^{\prime}}{(2 \pi)^{3}} \int \frac{\mathrm{d} \kappa_{1}}{(2 \pi)^{3}} \\
& \times\left\langle\chi^{(+)}\left(\boldsymbol{k}_{0}\right)\left|\left\langle 0|V| 1 \mu^{\prime}\right\rangle\right| \chi^{(-)}\left(\kappa_{1}^{\prime}\right)\right\rangle\left\langle\hat{\chi}^{(-)}\left(\kappa_{1}^{\prime}\right) \mid \hat{\chi}^{(+)}\left(\boldsymbol{k}_{1}^{\prime}\right)\right\rangle a_{\boldsymbol{1}^{\prime}}^{n^{\prime}} \frac{1}{E-E_{n^{\prime}}-E_{k_{1}^{\prime}}-\mathrm{i} \varepsilon} \\
& \times\left\langle\chi^{(+)}\left(\boldsymbol{k}_{1}^{\prime}\right)\left|\left\langle n^{\prime}|V| f\right\rangle\right| \chi^{(-)}(\boldsymbol{k})\right\rangle\left\langle\chi^{(-)}(\boldsymbol{k})|\langle f|V| n\rangle| \chi^{(+)}\left(\boldsymbol{k}_{1}\right)\right\rangle \frac{1}{E-E_{n}-E_{k_{1}}+\mathrm{i} \varepsilon} \\
& \times a_{1 \mu}^{n}\left\langle\hat{\chi}^{(+)}\left(\boldsymbol{k}_{1}\right) \mid \hat{\chi}^{(-)}\left(\kappa_{1}\right)\right\rangle\left\langle\chi^{(-)}\left(\kappa_{1}\right)|\langle 1 \mu|V| 0\rangle| \chi^{(+)}\left(\boldsymbol{k}_{0}\right)\right\rangle \delta\left(E_{f}-U\right) .
\end{aligned}
$$

Hence, by means of a simple operation the two-step process can be expressed in terms of two one-step matrix elements that are both of the normal DWBA type, at the expense of introducing an extra factor.

This extra factor is usually defined as the $S$-matrix. More specifically,

$$
S\left(\boldsymbol{k}^{\prime}, \boldsymbol{k}\right):=\left\langle\chi^{(-)}\left(\boldsymbol{k}^{\prime}\right) \mid \chi^{(+)}(\boldsymbol{k})\right\rangle \text {. }
$$


Similarly, for the complex conjugated distorted waves we define

$$
\hat{S}\left(\boldsymbol{k}^{\prime}, \boldsymbol{k}\right):=\left\langle\hat{\chi}^{(+)}\left(\boldsymbol{k}^{\prime}\right) \mid \hat{\chi}^{(-)}(\boldsymbol{k})\right\rangle .
$$

It is now easy to derive the relation between the two scattering matrices. With Eq. (2.37), we obtain

$$
\begin{aligned}
(2 \pi)^{3} \delta\left(\boldsymbol{k}-\boldsymbol{k}^{\prime}\right) & =\left\langle\chi^{(-)}(\boldsymbol{k}) \mid \hat{\chi}^{(-)}\left(\boldsymbol{k}^{\prime}\right)\right\rangle \\
& =\int \frac{\mathrm{d} \boldsymbol{k}^{\prime \prime}}{(2 \pi)^{3}}\left\langle\chi^{(-)}(\boldsymbol{k}) \mid \chi^{(+)}\left(\boldsymbol{k}^{\prime \prime}\right)\right\rangle\left\langle\hat{\chi}^{(+)}\left(\boldsymbol{k}^{\prime \prime}\right) \mid \hat{\chi}^{(-)}\left(\boldsymbol{k}^{\prime}\right)\right\rangle \\
& =\int \frac{\mathrm{d} \boldsymbol{k}^{\prime \prime}}{(2 \pi)^{3}} S\left(\boldsymbol{k}, \boldsymbol{k}^{\prime \prime}\right) \hat{S}\left(\boldsymbol{k}^{\prime \prime}, \boldsymbol{k}^{\prime}\right) .
\end{aligned}
$$

Hence, in the case of a potential with a non-vanishing imaginary part, the two scattering matrices are related by

$$
\hat{S}\left(\boldsymbol{k}^{\prime}, \boldsymbol{k}\right)=S^{-1}\left(\boldsymbol{k}, \boldsymbol{k}^{\prime}\right) .
$$

This relation is analogous to the standard relation $S^{\dagger}=S^{-1}$ that holds in the case of a real potential. Indeed, in the limit of a hermitian Hamiltonian, $\hat{S} \rightarrow S^{\dagger}$ and the normal unitarity condition $S^{\dagger}=S^{-1}$ is recovered (Feshbach, 1985).

From Eqs. (2.44) and (2.46) it follows that the transition amplitude (2.41) can be written as

$$
\begin{aligned}
T_{f \leftarrow 0}^{(2)}= & \sum_{n} \int \frac{\mathrm{d} \boldsymbol{k}_{1}}{(2 \pi)^{3}} \int \frac{\mathrm{d} \boldsymbol{k}_{1}^{\prime}}{(2 \pi)^{3}}\left\langle\chi^{(-)}(\boldsymbol{k})|\langle f|\boldsymbol{V}| n\rangle| \gamma^{(+)}\left(\boldsymbol{k}_{1}\right)\right\rangle \frac{1}{E-E_{n}-E_{k_{1}}+\mathrm{i} \varepsilon} \\
& \times S^{-1}\left(\boldsymbol{k}_{1}^{\prime}, \boldsymbol{k}_{1}\right)\left\langle\chi^{(-)}\left(\boldsymbol{k}_{1}^{\prime}\right)|\langle n|V| 0\rangle| \chi^{(+)}\left(\boldsymbol{k}_{0}\right)\right\rangle,
\end{aligned}
$$

and similarly the cross-section distribution (2.42) as

$$
\begin{aligned}
& \frac{\mathrm{d}^{2} \sigma^{(2)^{\mathrm{dh}}}}{\mathrm{d} \Omega \mathrm{d} E_{k}}=\frac{m^{2}}{\left(2 \pi \hbar^{2}\right)^{2}} \frac{k}{k_{0}} \sum_{f}\left|T_{f=0}^{(2)}\right|^{2} \delta\left(E_{f}-U\right) \\
& =\frac{m^{2}}{\left(2 \pi \hbar^{2}\right)^{2}} \frac{k}{k_{0}} \sum_{f} \sum_{n} \sum_{n^{\prime}} \sum_{\mu} \sum_{\mu^{\prime}} \int \frac{\mathrm{d} \boldsymbol{k}_{1}^{\prime}}{(2 \pi)^{3}} \int \frac{\mathrm{d} \boldsymbol{k}_{1}}{(2 \pi)^{3}} \int \frac{\mathrm{d} \boldsymbol{k}_{1}^{\prime}}{(2 \pi)^{3}} \int \frac{\mathrm{d} \kappa_{1}}{(2 \pi)^{3}} \\
& \times\left\langle\chi^{(+)}\left(\boldsymbol{k}_{0}\right)\left|\left\langle 0|V| 1 \mu^{\prime}\right\rangle\right| \chi^{(-\prime}\left(\kappa_{1}^{\prime}\right)\right\rangle S^{-1^{*}}\left(k_{1}^{\prime}, \boldsymbol{k}_{1}^{\prime}\right)\left|\hat{\chi}^{(+)}\left(\boldsymbol{k}_{1}^{\prime}\right)\right\rangle a_{1 \mu}^{\prime \prime} \frac{1}{E-E_{n^{\prime}}-E_{k_{1}}-\mathrm{i} \delta} \\
& \times\left\langle\chi^{(+)}\left(\boldsymbol{k}_{1}^{\prime}\right)\left|\left\langle n^{\prime}|V| f\right\rangle\right| \chi^{(-1}(\boldsymbol{k})\right\rangle\left\langle\chi^{(}{ }^{\prime}(\boldsymbol{k})|\langle f|V| n\rangle| \chi^{(+)}\left(\boldsymbol{k}_{1}\right)\right\rangle \frac{1}{E-E_{n}-E_{k_{1}}+\mathrm{i} \text { : }} \\
& \times a_{1 \mu}^{n} S^{-1}\left(\kappa_{1}, \boldsymbol{k}_{1}\right)\left\langle\chi^{(-1}\left(\kappa_{1}\right)|\langle 1 \mu|V| 0\rangle| \chi^{(+1}\left(\boldsymbol{k}_{0}\right)\right\rangle \delta\left(E_{j}-U\right) .
\end{aligned}
$$

Because the $S$-matrix elements are quite small in the case of strong absorption, the $S^{-1}$ factor can produce marked resonance behaviour in two-step processes. This anomalous behaviour of the two-step transition amplitude was first observed by Robson (1973). Moreover, the enhancement occurs at every subsequent step, eventually leading to a divergent perturbation series. 


\subsubsection{The apparent S-matrix singularity}

Austern and Vincent (1974) have pointed out that for the case of a single channel the apparent $S^{-1}$-singularity discovered by Robson is just an artifact of the representation. To illustrate this, we turn to a partial-wave expansion of $\chi^{(+)}$:

$$
\chi^{(+)}(k)=\frac{1}{k} \sum_{L}(2 L+1) \mathrm{i}^{L} \chi_{L}^{(+)}(k) P_{L}(\cos \Theta),
$$

where $\chi_{L}^{(+)}$now satisfies the radial Schrödinger equation. The partial distorted waves have asymptotically the form

$$
\chi_{L}^{(+)}(k) \rightarrow \frac{1}{r}\left[\mathrm{e}^{-\mathrm{i} k r}-S_{L}(k) \mathrm{e}^{\mathrm{i} k r}\right]
$$

which defines the partial scattering matrix $S_{L}$.

It can now be demonstrated that the general result (2.46) also holds in the partial-wave representation. To this end, we replace $k$ by $-k$ in Eq. (2.50), giving

$$
\begin{aligned}
\chi_{L}^{(+)}(-k) & \rightarrow \frac{1}{r}\left[e^{\mathrm{i} k r}-S_{L}(-k) \mathrm{e}^{-\mathrm{i} k r}\right] \\
& =\frac{1}{r}\left[-S_{L}(-k)\right]\left[\mathrm{e}^{-\mathrm{i} k r}-S_{L}^{-1}(-k) \mathrm{e}^{\mathrm{i} k r}\right] .
\end{aligned}
$$

Since the radial Schrödinger equation depends only on $k^{2}, \chi_{L}^{(+)}(k)$ and $\chi_{L}^{(+)}(-k)$ have the same dependence on $r$ so that

$$
S_{L}(k)=S_{L}^{-1}(-k) \text {. }
$$

The partial scattering matrix $\hat{S}_{L}$ is defined by the asymptotic condition for $\hat{\chi}^{(-)}$:

$$
\hat{\chi}_{L}^{(-)}(k) \rightarrow \frac{1}{r}\left[\mathrm{e}^{\mathrm{i} k r}-\hat{S}_{L}(k) \mathrm{e}^{-\mathrm{i} k r}\right] .
$$

Then, comparison with Eq. (2.51) shows that

$$
\hat{S_{L}}(k)=S_{L}(-k) \text {, }
$$

so that

$$
\hat{S}_{L}(k)=S_{L}^{-1}(k) .
$$

To complete the similar relations for the other functions, it can be deduced that

$$
\begin{aligned}
\chi_{L}^{(-)}(k) & =\chi_{L}^{(+)^{*}}(-k) \\
& \rightarrow \frac{1}{r}\left[\mathrm{e}^{-\mathrm{i} k r}-S_{L}(-k) \mathrm{e}^{\mathrm{i} k r}\right] \\
& =\frac{1}{r}\left[-S_{L}(-k)\right]\left[\mathrm{e}^{\mathrm{i} k r}-S_{L}^{-1}(-k) \mathrm{e}^{-\mathrm{i} k r}\right] \\
& =\frac{1}{r}\left[-S_{L}(-k)\right]\left[\mathrm{e}^{\mathrm{i} k r}-S_{L}(k) \mathrm{e}^{-\mathrm{i} k r}\right]
\end{aligned}
$$


and

$$
\begin{aligned}
\hat{\chi}_{I .}^{(+)}(k) & =\hat{\chi}_{L}^{(-)^{*}}(-k) \\
& \rightarrow \frac{1}{r}\left[\mathrm{e}^{i k r}-\hat{S}_{L}(-k) \mathrm{e}^{\mathrm{i} k r}\right] \\
& =\frac{1}{r}\left[-\hat{S}_{L}(-k)\right]\left[\mathrm{e}^{-i k r}-\hat{S}_{I}{ }^{1}(-k) \mathrm{e}^{\mathrm{i} k r}\right] \\
& =\frac{1}{r}\left[-\hat{S}_{I}(-k)\right]\left[\mathrm{e}^{-i k r}-\hat{S}_{I}(k) \mathrm{e}^{i k r}\right] .
\end{aligned}
$$

To summarise, we have the following asymptotic conditions for the partial distorted waves:

$$
\begin{aligned}
& \chi_{L}^{(+)}(k) \rightarrow \frac{1}{r}\left(\mathrm{e}^{-i k r}-S_{L}(k) \mathrm{e}^{\mathrm{i} k r}\right) \\
& \hat{\chi}_{L}^{(+)}(k) \rightarrow \frac{1}{r}\left(\mathrm{e}^{-i k r}-S_{I}{ }^{1}(k) \mathrm{e}^{\mathrm{i} k r}\right) \\
& \chi_{L}^{(-)}(k) \rightarrow \frac{1}{r}\left(\mathrm{e}^{\mathrm{i} k r}-S_{L}(k) \mathrm{e}^{\mathrm{i} k r}\right) \\
& \hat{\chi}_{L}^{(-)}(k) \rightarrow \frac{1}{r}\left(\mathrm{e}^{\mathrm{i} k r}-S_{L}^{-1}(k) \mathrm{e}^{-i k r}\right) .
\end{aligned}
$$

and finally we find

$$
\begin{aligned}
\hat{\chi}_{L}^{(+)}(k) & \rightarrow \frac{1}{r}\left(\mathrm{e}^{-\mathrm{i} k r}-S_{L}^{-1}(k) \mathrm{e}^{\mathrm{i} k r}\right) \\
& =-\frac{1}{r} S_{L}^{-1}(k)\left(\mathrm{e}^{\mathrm{j} k r}-S_{L}(k) \mathrm{e}^{i k r}\right) \\
& =-S_{L^{\prime}}{ }^{1}(k) \chi_{L}^{(-1}(k) .
\end{aligned}
$$

Hence, in the partial wave expansion we can, analogous to Eq. (2.47), replace $\hat{\chi}_{1}^{1+}$ ' by $\chi_{L}^{\prime}$ ', 'but then we also introduce an additional factor that is the reciprocal of $S_{L}$.

Given these relations, it can now be shown that the $S^{-1}$-singularity in the transition amplitude is only apparent (Austern and Vincent, 1974; Feshbach, 1992) and that there is no undesirable enhancement of the multistep amplitude. To see this, consider the s-wave component of the radial distorted waves Green function $G\left(r, r^{\prime}\right)$ for a single channel. For $r$ and $r^{\prime}$ outside the interaction range

$$
\begin{aligned}
G_{L=0}^{\text {outside }}\left(r, r^{\prime}\right) & =\frac{2}{\pi} \int_{0}^{\infty} \mathrm{d} k \frac{\left[\mathrm{e}^{-\mathrm{i} k r}-S_{0}(k) \mathrm{e}^{\mathrm{i} k r}\right]\left[\mathrm{e}^{-\mathrm{i} k r^{\prime}}-S_{0}(k) \mathrm{e}^{\mathrm{i} k r^{\prime}}\right]}{S_{0}(k)\left(E-\hbar^{2} k^{2} / 2 m+\mathrm{i} \varepsilon\right)} \\
& =-\frac{2}{\pi} \int_{0}^{\infty} \mathrm{d} k \frac{\mathrm{e}^{\mathrm{i} k\left(r-r^{\prime}\right)}+\mathrm{e}^{\mathrm{ik(r-r^{ \prime } )}-S_{0}(k) \mathrm{e}^{\mathrm{i} k\left(r+r^{\prime}\right)}-S_{0}^{-1}(k) \mathrm{e}^{-\mathrm{i} k\left(r+r^{\prime}\right)}}}{E-\hbar^{2} k^{2} / 2 m+\mathrm{i} \varepsilon}
\end{aligned}
$$


The last term can be handled by changing the integration variable from $k$ to $-k$ and using Eq. (2.52). Then,

$$
\begin{aligned}
\int_{0}^{\infty} \mathrm{d} k \frac{\mathrm{e}^{\mathrm{i} k\left(r+r^{\prime}\right)}}{S_{0}(k)\left(E-\hbar^{2} k^{2} / 2 m+\mathrm{i} \varepsilon\right)} & =\int_{-\infty}^{0} \mathrm{~d} k \frac{\mathrm{e}^{\mathrm{i} k\left(r+r^{\prime}\right)}}{S_{0}(-k)\left(E-\hbar^{2} k^{2} / 2 m+\mathrm{i} \varepsilon\right)} \\
& =\int_{-\infty}^{0} \mathrm{~d} k \frac{S_{0}(k) \mathrm{e}^{\mathrm{i} k\left(r+r^{\prime}\right)}}{E-\hbar^{2} k^{2} / 2 m+\mathrm{i} \varepsilon} .
\end{aligned}
$$

This can be combined with the third term of Eq. (2.60) to yield

$$
G_{L=0}^{\text {outside }}\left(r, r^{\prime}\right)=-\frac{2}{\pi}\left[\int_{0}^{\infty} \mathrm{d} k \frac{\mathrm{e}^{\mathrm{i} k\left(r-r^{\prime}\right)}+\mathrm{e}^{-\mathrm{i} k\left(r-r^{\prime}\right)}}{E-\hbar^{2} k^{2} / 2 m+\mathrm{i} \varepsilon}-\int_{-\infty}^{\infty} \mathrm{d} k \frac{S_{0}(k) \mathrm{e}^{\mathrm{i} k\left(r+r^{\prime}\right)}}{E-\hbar^{2} k^{2} / 2 m+\mathrm{i} \varepsilon}\right],
$$

which is no longer divergent. The first term is clearly convergent and also the second one can be readily evaluated by closing the integration contour in the upper half complex $k$-plane. The only additional requirement is that $S_{0}(k)$ has appropriate behaviour for $|k| \rightarrow \infty$. The conclusion is that for the single channel case, there is no creativity problem related to the regenerative optical potential.

\subsubsection{S-matrix poles for a regenerative Hamiltonian}

The foregoing immediately implies that great care is required with the analysis of the manychannel case. It is imperative that the effects of the $S^{-1}$-factor are properly eliminated before statistical assumptions with respect to the continuum of levels are invoked. Otherwise, the phase relations among the different partial wave components, which would normally cancel each other for a single channel, are neglected and an unphysical enhancement of the multistep terms will occur.

To elucidate the energy averaging procedure that has been suggested by Feshbach (1985) to solve this problem, it is instructive to investigate the properties of the $S$-matrix poles, in the complex $k$-plane, in the presence of an imaginary potential (Joffily, 1973; Gal et al., 1981; Cassing and Stingl, 1982).

First consider a purely real potential, see Fig. 2. Poles of the $S$-matrix may occur on the positive imaginary $k$-axis. These are the bound state poles (open circles) corresponding to a negative energy. On the negative imaginary $k$-axis there may exist virtual state poles (full circles). Finally, possible resonance poles (crosses) are symmetrically placed with respect to the negative imaginary $k$-axis.

Upon a gradual introduction of an absorptive potential (see Fig. 3), the bound state poles rotate counterclockwise into the second quadrant, the resonance poles of the fourth quadrant move away (clockwise rotation) from the positive real $k$-axis whereas their mirror poles in the third quadrant move towards the negative real $k$-axis. For a sufficiently strong absorption, some poles may end up in the second quadrant of the complex $k$-plane. The nature of the latter poles becomes clear if we look at the asymptotic wavefunction (Joffily, 1973) for values of $k=k_{1}+i k_{2}$ that correspond to zeros of $S_{L}(k)$, i.e. to poles of $S_{L}(-k)$

$$
\begin{aligned}
\lim _{r \rightarrow \infty} \psi_{L}(r, t) \sim & \frac{1}{2 \mathrm{i} k r} P_{L}(\cos \theta)(2 L+1) \exp \left\{-\mathrm{i}\left[k_{1} r+\left(k_{1}^{2}-k_{2}^{2}\right) \frac{\hbar}{2 m} t\right]\right\} \\
& \times \exp \left(k_{1} k_{2} \frac{\hbar}{m} t\right) \exp \left(k_{2} r\right) .
\end{aligned}
$$




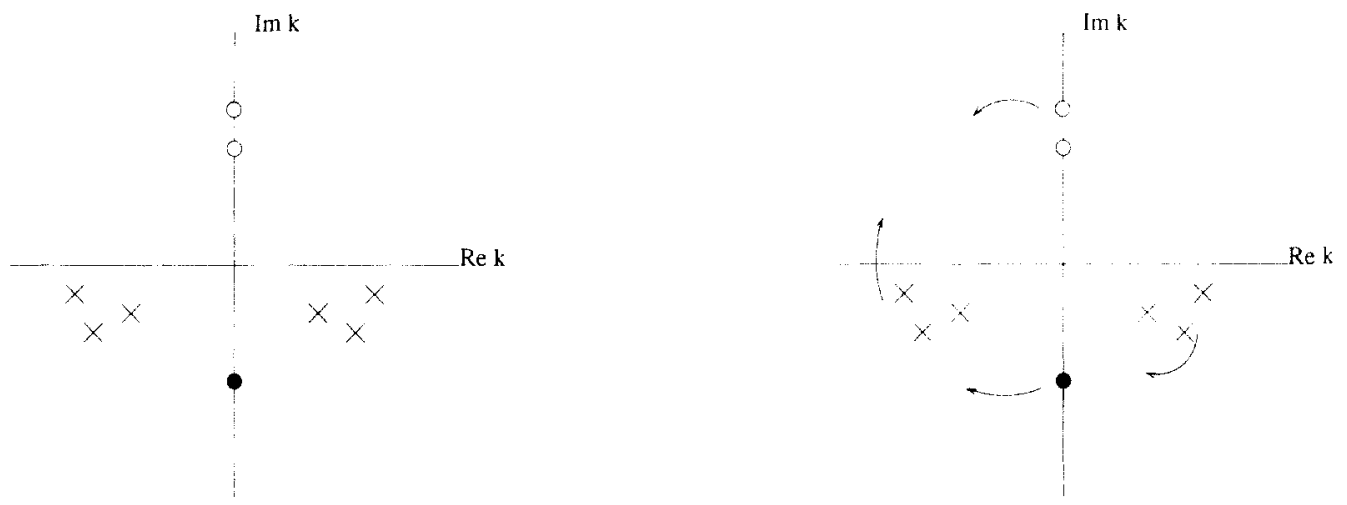

Fig. 2. Poles of the $S$-matrix in the complex $k$-plane for a real potential. The bound state poles, virtual state poles and resonance poles are designated by open circles, full circles and crosses, respectively.

Fig. 3. Motion of poles of the $S$-matrix in the complex $k$-plane for an absorptive potential. The bound state poles, virtual state poles and resonance poles are designated by open circles, full circles and crosses, respectively.

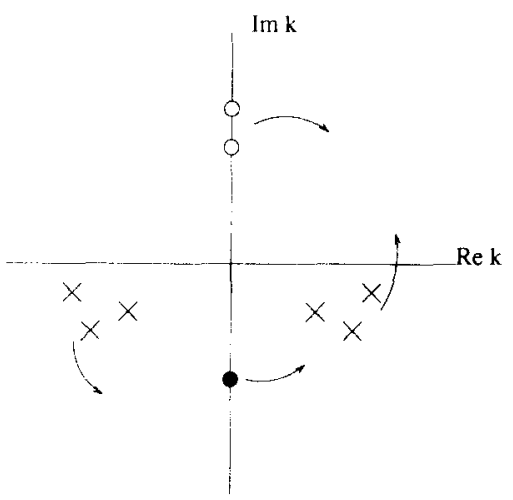

Fig. 4. Motion of poles of the $S$-matrix in the complex $k$-plane for a regenerative potential. The bound state poles, virtual state poles and resonance poles are designated by open circles, full circles and crosses, respectively.

With this equation it can be seen that the poles that moved into the second quadrant correspond to an incoming wavefunction exponentially decaying in time. These wavefunctions are called bound states in the continuum.

The distorted waves $\hat{\chi}^{(+)}$, however, are eigenfunctions of a regenerative Hamiltonian, and upon introduction of a regenerative optical potential, the poles of the $S$-matrix of the lower $k$-plane move counterclockwise, as shown in Fig. 4. This time, for a sufficiently strong regenerative potential the poles can move into the first quadrant. Consulting Eq. (2.63) again tells us that the corresponding wavefunctions are of an outgoing wave nature and grow exponentially in time. Hence, the causality condition that $S$-matrix poles cannot occur in the first quadrant seems to be violated in the 
presence of a regenerative optical potential. It leads to the strange phenomenon that particle flux is being created by the scattering centre.

The presence of these scattering resonances suggests that one must energy average $\hat{\chi}^{(+)}$in order to get an appropriate optical model wavefunction and optical model Hamiltonian. The wavefunction $\hat{\chi}^{(+)}$is the elastic channel component of the total wavefunction $\Psi$ satisfying

$$
H^{\dagger} \Psi=E \Psi
$$

where $\mathrm{H}^{\dagger}$ is the regenerative Hamiltonian, which is energy-averaged over the fluctuations due to the non-elastic channels. It operates in the subspace that contains only open channels, and these are isolated by the projection operator $P$

$$
P \Psi=\hat{\chi} .
$$

The remaining channels are projected by the operator $Q=1-P$. This gives the familiar equations

$$
\begin{aligned}
& \left(E-H_{P P}^{\dagger}\right) \hat{\chi}^{(+)}=H_{P Q}^{\dagger}(Q \Psi), \\
& \left(E-H_{Q Q}^{\dagger}\right) Q \Psi=H_{Q P}^{\dagger} \hat{\chi}^{(+)}
\end{aligned}
$$

and hence

$$
\left(E-H_{n}^{\dagger}\right) \hat{\chi}^{(+)}=0,
$$

where the effective regenerative Hamiltonian is

$$
H_{n}^{\dagger}=H_{P P}^{\dagger}+H_{P Q}^{\dagger} \frac{1}{E-H_{Q Q}^{\dagger}} H_{Q P}^{\dagger} .
$$

The first term connects directly the initial and final states and the second term represents the virtual excitations of states in the compound nucleus and the coupling to non-elastic channels and is responsible for the energy fluctuations of $\hat{\chi}^{(+)}$.

The $S$-matrix poles in the $E$-plane show a behaviour similar to those in the $k$-plane. However, since the mapping of $k$ to $E$ is two-to-one, $S_{L}(E)$ is a function on a two-sheeted Riemann surface. The upper half of the $k$-plane $(\operatorname{Im} k>0)$ corresponds to the physical sheet and the lower half $(\operatorname{Im} k<0)$ corresponds to the second sheet. To preserve analyticity, a cut must be put along the real $E$-axis from 0 to $\infty$. The resonance zero of the $S$-matrix in the fourth quadrant close to the real $k$-axis (as in Fig. 3) becomes a zero on the second sheet of the $E$-plane, close to the physical region, at $E=E_{R}-\mathrm{i} \Gamma / 2$.

In the present context, we are primarily concerned with resonances that are associated with eigenvalues of the regenerative Hamiltonian $H_{Q Q}^{\dagger}$. As can be inferred from Fig. 5, some of these poles are in the first quadrant of the physical sheet of the $E$-plane and this time the corresponding eigenvalues have a positive imaginary part, i.e.

$$
E_{v}+\frac{\mathrm{i} \Gamma_{v}}{2}
$$

These poles correspond to resonances in $\hat{\chi}^{(+)}$with widths $\left(\Gamma_{v}^{\dagger}-\Gamma_{v}\right)$, where $\Gamma_{v}^{\dagger}$ is the escape width and $\Gamma_{v}$ is the total width. 


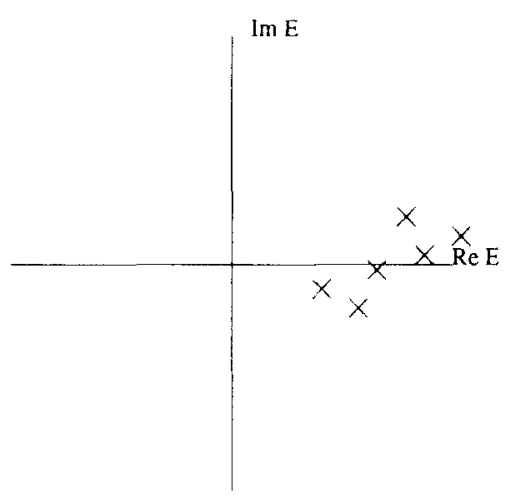

(a)

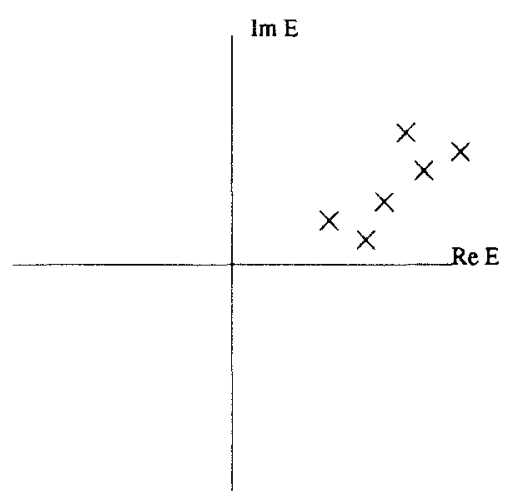

(b)

Fig. 5. Resonance poles of the $S$-matrix in the complex $E$-plane (a) before energy averaging and (b) after energy averaging.

We note that $\hat{\chi}^{(+)}$has the formal solution

$$
\hat{\chi}^{(+)}=\phi+\frac{1}{E-H_{n}^{\dagger}+\mathrm{i} \varepsilon} V_{n}^{\dagger} \phi .
$$

Analogous to the derivation of the optical model (Feshbach, 1962; Feshbach et al. 1967; Austern, 1970 ) one should now energy average $Q \Psi$ and hence also the effective regenerative Hamiltonian (2.68). It is assumed that the bound states on the first quadrant of the physical sheet of the $E$-plane, which are localized in the interaction region, make the most significant contribution to the propagator $1 /\left(E-H_{n}^{\dagger}\right)$. Hence, we replace the propagator by its spectral sum over the bound states only

$$
\frac{1}{E-H_{n}^{\dagger}}=\sum_{v} \frac{\left|\psi_{v}\right\rangle\left\langle\psi_{v}\right|}{E-E_{v}+(\mathrm{i} / 2)\left(\Gamma_{v}^{\dagger}-\Gamma_{v}\right)} .
$$

where the $\psi_{v}$ are the bound-state wavefunctions. These wavefunctions decay exponentially so there is no need to retain the ic to specify the incoming or outgoing boundary condition.

The corresponding resonance poles of the $S$-matrix in the E-plane are depicted in Fig. 5(a). In this figure, we have restricted ourselves to the poles that are relevant in the present context. These do not only include the poles that are in the first quadrant of the physical sheet but also the poles in the fourth quadrant of the second sheet that happen to be close to the real $E$-axis.

\subsubsection{Energy averaging}

The crucial step in the derivation is to perform an energy average over an energy interval $I / 2$. Then, referring to the well-known relation $\bar{f}(E)=f(E+\mathrm{i} I)$, the width $\Gamma_{v}$ is increased by $I$ and for the energy-averaged propagator we have

$$
\left\langle\frac{1}{E-H_{n}^{\dagger}}\right\rangle_{E}=\frac{1}{E-\bar{H}_{n}^{\dagger}}=\sum_{v} \frac{\left|\psi_{v}\right\rangle\left\langle\psi_{v}\right|}{E-E_{v}+(\mathrm{i} / 2)\left(\Gamma_{v}^{\dagger}-\Gamma_{v}-I\right)} .
$$




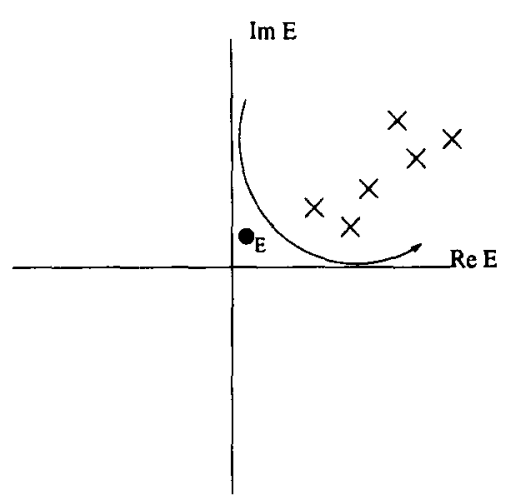

(a)

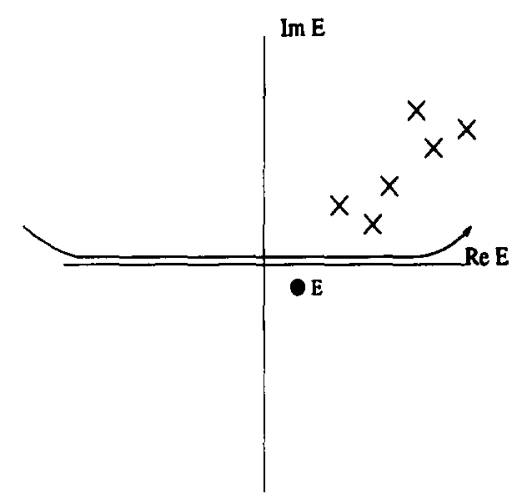

(b)

Fig. 6. (a) Cauchy contour for integral representation of the energy-averaged propagator, (b) deformation of the contour to the real $E$-axis.

The averaging interval $I$ is chosen so that for all the poles that contribute significantly to the cross-section, $\Gamma_{v}^{\uparrow}-\Gamma_{v}-I$ is negative for all $v$. This has the effect of moving all poles into the first quadrant, see Fig. 6(b).

Also, in first approximation we have for the wavefunction $\left\langle\hat{\chi}^{(+)}\right\rangle_{E}$

$$
\begin{aligned}
\left\langle\hat{\chi}^{(+)}\right\rangle_{E} & =\phi+\left\langle\frac{1}{E-H_{n}^{\dagger}+\mathrm{i} \varepsilon} V_{n}^{\dagger}\right\rangle_{E} \phi \\
& =\phi+\frac{1}{E-\bar{H}_{n}^{\dagger}} \bar{V}_{n}^{\dagger} \phi,
\end{aligned}
$$

where it is assumed that $\phi$ and $V_{n}^{\dagger}$ are weakly energy dependent.

By choosing $I$ large enough, we have attained that the imaginary part of the denominator of Eq. (2.72) is negative, which already indicates the obtained incoming boundary condition upon the energy averaging. To complete the analysis and returning to the continuum representation, we write the energy-average propagator as a contour integral enclosing the poles

$$
\frac{1}{E-\bar{H}_{n}^{\dagger}}=-\frac{1}{2 \pi \mathrm{i}} \oint \frac{\mathrm{d} z}{(E-z)\left(z-\bar{H}_{n}^{\dagger}\right)} .
$$

This integral yields the obtained result (2.72) provided the energy $E$ is outside the contour, see Fig. 6(a). To evaluate this integral we deform the contour so that it runs along the real $E$-axis and is completed by a semi-circle at infinity, see Fig. $6($ b). The requirement that the energy $E$ must be outside the contour means that it must lie below the real $E$-axis, i.e. $E \rightarrow E-\mathrm{i} \varepsilon$. This yields for the propagator

$$
\left\langle\frac{1}{E-\bar{H}_{n}^{\dagger}}\right\rangle_{E}=\frac{1}{E-\bar{H}_{n}^{\dagger}-\mathrm{i} \varepsilon}
$$


and for Eq. (2.73)

$$
\begin{aligned}
\left\langle\hat{\chi}^{(+)}\right\rangle_{E} & =\phi+\frac{1}{E-\bar{H}_{n}^{\dagger}-\mathrm{i} \varepsilon} \bar{V}_{n}^{\dagger} \phi \\
& \rightarrow \bar{\chi}^{(-)},
\end{aligned}
$$

where the bar over the wavefunction denotes the energy average. For the absorptive potential, the poles are in the fourth quadrant of the $E$-plane, and for the energy average of $\chi^{(+)}$we find that $E \rightarrow E+\mathrm{i} \varepsilon$, i.e. it approaches the real $E$-axis from above. Thus

$$
\begin{aligned}
\left\langle\chi^{(+)}\right\rangle_{E} & =\phi+\frac{1}{E-\bar{H}_{n}+\mathrm{i} \varepsilon} \bar{V}_{n} \phi \\
& \rightarrow \bar{\chi}^{(+)}
\end{aligned}
$$

The inverse $S$-matrix elements of Eq. (2.42) can now be eliminated, because with Eq. (2.76) we have

$$
\begin{aligned}
\left\langle\left\langle\hat{\chi}^{(+)}\left(\boldsymbol{k}_{1} \mid \hat{\chi}^{(-)}(\kappa)_{1}\right)\right\rangle\right\rangle_{E} & =\left\langle\bar{\chi}^{(-)}\left(\boldsymbol{k}_{1}\right) \mid \bar{\chi}^{(-)}\left(\kappa_{1}\right)\right\rangle \\
& =(2 \pi)^{3} \delta\left(\boldsymbol{k}_{1}-\kappa_{1}\right),
\end{aligned}
$$

and hence, we obtain for the two-step cross-section distribution

$$
\begin{aligned}
\frac{\mathrm{d}^{2} \sigma^{(2)^{\mathrm{dis}^{\mathrm{is}}}}=}{\mathrm{d} \Omega \mathrm{d} E_{k}}= & \frac{2 m^{5}}{(2 \pi)^{8} \hbar^{10}} \frac{k}{k_{0}} \sum_{f} \sum_{n} \sum_{n^{\prime}} \sum_{\mu} \sum_{\mu^{\prime}} \int \mathrm{d} \Omega_{1}^{\prime} \int \mathrm{d} \Omega_{1} \int \mathrm{d} E_{k_{1}^{\prime}} E_{k_{i}^{\prime}}^{1 / 2} \int \mathrm{d} E_{k_{1}} E_{k_{1}}^{1 / 2} \\
& \times\left\langle\bar{\chi}^{(+)}\left(E_{k_{0}}, \Omega_{0}\right)\left|\left\langle 0|V| 1 \mu^{\prime}\right\rangle\right| \bar{\chi}^{(-)}\left(E_{k_{1}^{\prime}}, \Omega_{1}^{\prime}\right)\right\rangle a_{1 \mu^{\prime}}^{n^{\prime}} \frac{1}{E-E_{n^{\prime}}-E_{k_{1}}-\mathrm{i} \varepsilon} \\
& \times\left\langle\bar{\chi}^{(+)}\left(E_{k_{1}^{\prime}}, \Omega_{1}^{\prime}\right)\left|\left\langle n^{\prime}|V| f\right\rangle\right| \bar{\chi}^{(-)}\left(E_{k}, \Omega\right)\right\rangle\left\langle\bar{\chi}^{(-)}\left(E_{k}, \Omega\right)|\langle f|V| n\rangle| \bar{\chi}^{(+)}\left(E_{k_{1}}, \Omega_{1}\right)\right\rangle \\
& \times a_{1 \mu}^{n} \frac{1}{E-E_{n}-E_{k_{1}}+\mathrm{i} \varepsilon}\left\langle\bar{\chi}^{(-)}\left(E_{k_{1}}, \Omega_{1}\right)|\langle 1 \mu|V| 0\rangle| \bar{\chi}^{(+)}\left(E_{k_{0}}, \Omega_{0}\right)\right\rangle \delta\left(E_{f}-U\right) .
\end{aligned}
$$

Obviously, the extra energy average as suggested by Feshbach has resulted in a two-step expression consisting entirely of normal DWBA matrix elements.

\subsection{Statistics}

So far, all quantum interference effects of the residual nuclear states and the leading particle have been retained in the cross-section formulae. It is clear that these expressions for continuum reactions are highly complicated and that it is virtually impossible to perform individual crosssection calculations in regions with a high level density. It is therefore inevitable that statistical assumptions are introduced in order to remove interference effects so that a relatively simple expression for the energy-averaged cross-section is obtained. It has been stressed by Koning and Akkermans (1991) that there are two different types of statistics, each originating from a different part of the total Hamiltonian (2.17). 


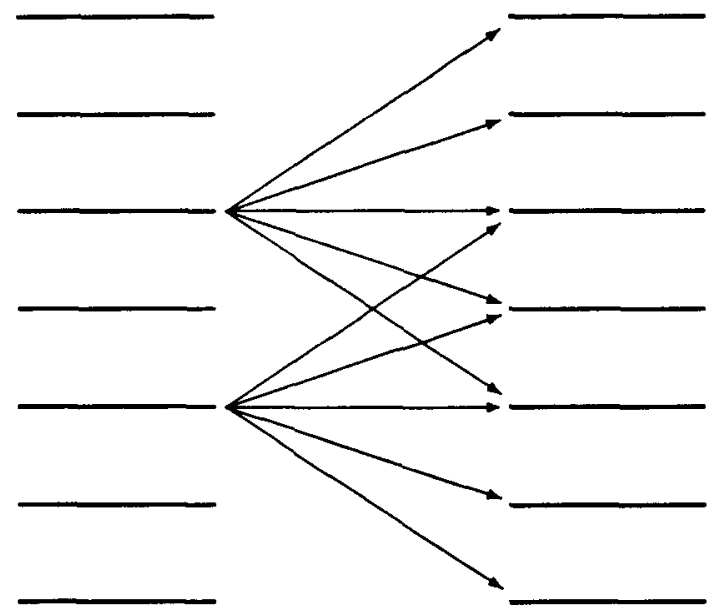

Fig. 7. Leading-particle statistics: many states can be created in a random manner by the leading particle from a given state.

If randomness is assigned to the residual interaction of the residual nucleus $H_{1}$, we speak of residual-system statistics. Formally this type of statistics is expressed by means of random configuration mixing, i.e. the distribution amplitudes $a_{m \mu}^{n}$ of the linear combination (2.23) are assumed to be random

$$
\overline{\sum_{n} a_{m^{\prime} \mu^{\prime}}^{n} a_{m \mu}^{n} \delta\left(E_{n}-E_{x}\right)}=\delta_{m m^{\prime}} \delta_{\mu \mu^{\prime}} \overline{\sum_{n}\left|a_{m \mu}^{n}\right|^{2} \delta\left(E_{n}-E_{x}\right)}
$$

Residual-system statistics is a feature of the residual nucleus alone and has nothing to do with interaction of the incident particle with the residual nucleus. It lies at the basis of the multistep direct theories of Tamura et al. (1982) (TUL) and of Nishioka et al. (1988) (NWY). In this review we will not be concerned with these models.

The type of statistics which is of particular interest to the FKK model is called leading-particle statistics. Random properties are assigned to the interaction $V$ of the leading particle with the residual nucleus. It is supposed that $V$ connects a given nuclear state to many other nuclear states (as depicted in Fig. 7), and that the associated set of matrix elements is randomly distributed. Formally, it is expressed by

$$
\begin{aligned}
& \overline{\left\langle\chi^{(+)}\left(E_{k_{0}}, \Omega_{0}\right)\left|\left\langle 0|V| 1 \mu^{\prime}\right\rangle\right| \chi^{(-)}\left(E_{k}^{\prime}, \Omega^{\prime}\right)\right\rangle\left\langle\chi^{(-)}\left(E_{k}, \Omega\right)|\langle 1 \mu|V| 0\rangle| \chi^{(+)}\left(E_{k_{0}}, \Omega_{0}\right)\right\rangle} \\
& =\delta_{\mu \mu^{\prime}} \overline{\left.\left\langle\chi^{(-)}\left(E_{k}, \Omega\right)|\langle 1 p 1 h|V| 0\rangle| \chi^{(+)}\left(E_{k_{0}}, \Omega_{0}\right)\right\rangle\right|^{2}} .
\end{aligned}
$$

Here, the bar denotes the ensemble average. Observe that the squared matrix element on the right does not depend on the index $\mu$. This is directly associated with the assumption that the ensemble average is equal to the average over energy. The critical assumption here is that non-diagonal cross-products of matrix elements cancel upon energy averaging, because these matrix elements vary widely both in magnitude and sign and therefore can be considered to be random variables. 
In the original derivation of the FKK model, both the randomness of the distribution amplitudes and the matrix elements are employed. Koning and Akkermans (1991) have demonstrated that leading-particle statistics, i.e. the randomness of the matrix elements, is a necessary and sufficient condition for the convolution-type structure of the FKK cross section.

In the following, we will demonstrate how the FKK one- and multistep cross-section follows by applying leading-particle statistics only.

\subsection{The one-step cross-section}

The cross-section distribution for the first step given by Eq. (2.31) can be averaged over the final energy using leading-particle statistics [Eq. (2.81)]. Using a decoupled energy-solid angle notation $\left(E_{k}, \Omega\right)$ instead of the momentum-vector $(\boldsymbol{k})$ gives for the energy-averaged one-step cross-section

$$
\begin{aligned}
\frac{\mathrm{d}^{2} \sigma^{(1)}}{\mathrm{d} \Omega \mathrm{d} E_{k}} & =\frac{m^{2}}{\left(2 \pi \hbar^{2}\right)^{2}} \frac{k}{k_{0}} \sum_{f} \sum_{\mu} \overline{\left|a_{1 \mu}^{f}\right|^{2}\left|\left\langle\chi^{(-)}\left(E_{k}, \Omega\right)|\langle 1 p 1 h|V| 0\rangle| \chi^{(+)}\left(E_{k_{0}}, \Omega_{0}\right)\right\rangle\right|^{2} \delta\left(E_{f}-U\right)} \\
& =\frac{m^{2}}{\left(2 \pi h^{2}\right)^{2}} \frac{k}{k_{0}} \sum_{\mu} \hat{\rho}_{1 \mu}(U) \mid \overline{\left.\left\langle\chi^{(-)}\left(E_{k}, \Omega\right)|\langle 1 p 1 h|V| 0\rangle| \chi^{(+)}\left(E_{k_{0}}, \Omega_{0}\right)\right\rangle\right|^{2}}
\end{aligned}
$$

where the true particle-hole state density $\hat{\rho}_{m \mu}$ is defined by

$$
\hat{\rho}_{m \mu}(U)=\sum_{f} \overline{\left|a_{m \mu}^{f}\right|^{2} \delta\left(E_{f}-U\right)} .
$$

The adjective true designates the configuration mixing of the particle-hole states over the real states of the residual nucleus (Tamura et al. 1982). It is imagined that around each particle-hole state a probability distribution $\sum_{f}\left|a_{m \mu}^{f}\right|^{2} \delta\left(E_{f}-U\right)$ is given, its width being a measure for the magnitude of the residual interaction of the residual nucleus.

Then, the contribution of each particle-hole state to a real state is represented by the probability distribution at the considered energy of the real state.

Having obtained an energy-averaged cross-section, we can decompose it into different transferred angular momenta $J$ to give an expression in terms of first-order DWBA cross-sections, see Eq. (2.6), averaged per angular momentum

$$
\frac{\mathrm{d}^{2} \sigma^{(1)}}{\mathrm{d} \Omega \mathrm{d} E_{k}}=\sum_{j} \hat{\rho}_{1 p 1 h, J}(U)\left[\frac{\mathrm{d} \sigma^{(1)}\left(E_{k} \leftarrow E_{k_{0}}\right)}{\mathrm{d} \Omega}\right]_{J}^{\mathrm{DWBA}},
$$

where the true state density has now been averaged over the particle-hole states for each angular momentum

$$
\hat{\rho}_{1 p 1 h, J}(U)=\left\langle\hat{\rho}_{1 \mu}(U)\right\rangle_{\mu, J} .
$$

In practical calculations, one often disregards the aforementioned configuration mixing effects. Rather, independent particle model state densities, $\rho_{1 p 1 h, J}(U)$, are employed. These may formally be obtained from the true state densities by the limit $a_{m \mu}^{f} \rightarrow \delta_{m \mu} f$. A well-known approximation is

$$
\rho_{1 p 1 h, J}(U)=(2 J+1) R_{2}(J) \omega_{1 p 1 h}(U) .
$$


where the particle-hole state density for $p$ particles and $h$ holes is given by Williams' formula (1971)

$$
\omega_{p, h}(U)=g^{n} \frac{\left(U-A_{p, h}-P\right)^{n-1}}{p ! h !(n-1) !},
$$

where $g$ is the single-particle state density, $U$ the excitation energy, $n=p+h$ the exciton number, $A_{p, h}=[p(p-1)+h(h-1)] / 4 g$ the Pauli correction factor and $P$ the pairing correction. The function $R_{n}(J)$ (with spin cut-off factor $\sigma$ ) represents the spin distribution of the residual states in the continuum. It is given by

$$
R_{n}(J)=\frac{2 J+1}{\pi^{1 / 2} n^{3 / 2} \sigma^{3}} \exp \left[-\frac{\left(J+\frac{1}{2}\right)^{2}}{n \sigma^{2}}\right]
$$

A more general prescription for the particle-hole state density has been given by Obložinský (1986) [see also Běták and Dobeš (1976)]. This expression contains a correction for the finite hole depth and applies at high excitation energies (above the Fermi energy)

$$
\omega_{p, h}(U)=\frac{g^{n}}{p ! h !(n-1) !} \sum_{j=0}^{h}(-1)^{j}\left(\begin{array}{l}
h \\
j
\end{array}\right)\left(U-P-A_{p, h}-j F\right)^{n-1} \Theta\left(U-P-\alpha_{p, h}-j F\right) .
$$

Here, $F$ is the Fermi energy, $\alpha_{p, h}=\left(p^{2}+h^{2}+p-h\right) / 2 g$, and $\Theta$ is the unit step function. If the incident nucleon energy exceeds about $50 \mathrm{MeV}$ and one is interested in the multistep direct contribution at low outgoing energies, Eq. (2.89) instead of the conventional Williams expression (2.87) should be used in the calculation.

Hence, the FKK one-step cross-section is obtained by computing Eq. (2.84) together with Eqs. (2.86) and (2.88) using Eq. (2.87) or Eq. (2.89).

\subsection{The two-step cross-section}

Previously we discussed how the two-step process can be expressed in terms of two normal DWBA matrix elements by means of energy averaging. The next step is to apply the statistical hypothesis (2.81) to this two-step cross-section distribution to obtain the FKK cross-section. In the fast multistep direct process, an obvious strategy is the introduction of the so-called never-comeback approximation, that is, to assume that in each subsequent step of the reaction a new particle-hole pair is created. We note that the never-come-back approximation was not used by Feshbach et al. (1980). This standard simplification is however justified since in the early steps particle-hole creation strongly dominates over other processes such as particle-hole annihilation or exchange. Formally

$$
\langle f|V| n\rangle=\sum_{v} a_{(1 v) n}^{f}\langle 1 v, n|V| n\rangle
$$


Inserting this in Eq. (2.79) gives

$$
\begin{aligned}
\frac{\mathrm{d}^{2} \sigma^{(2)^{\mathrm{dis}}}}{\mathrm{d} \Omega \mathrm{d} E_{k}}= & \frac{2 m^{5}}{(2 \pi)^{8} \hbar^{10}} \frac{k}{k_{0}} \sum_{f} \sum_{n} \sum_{n^{\prime}} \sum_{v} \sum_{v^{\prime}} \sum_{\mu} \sum_{\mu^{\prime}} \int \mathrm{d} \Omega_{1}^{\prime} \int \mathrm{d} \Omega_{1} \int \mathrm{d} E_{k_{1}^{\prime}} E_{k^{\prime}}^{1 / 2} \int \mathrm{d} E_{k_{1}^{\prime}} E_{k_{1}^{\prime}}^{1 / 2} \\
& \times a_{\left(1 v^{\prime}\right) n^{\prime}}^{f} a_{(1, y)}^{f}\left\langle\bar{\chi}^{(+)}\left(E_{k_{0}}, \Omega_{0}\right)\left|\left\langle 0|V| 1 \mu^{\prime}\right\rangle\right| \bar{\chi}^{(-)}\left(E_{k_{1}^{\prime}}, \Omega_{1}^{\prime}\right)\right\rangle a_{1 \mu^{\prime}}^{n^{\prime}} \frac{1}{E-E_{n^{\prime}}-E_{k_{1}^{\prime}}-\mathrm{i} \varepsilon} \\
& \times\left\langle\bar{\chi}^{(+)}\left(E_{k_{1}^{\prime}}, \Omega_{1}^{\prime}\right)\left|\left\langle n^{\prime}|V| 1 v^{\prime}, n^{\prime}\right\rangle\right| \bar{\chi}^{(-)}\left(E_{k}, \Omega\right)\right\rangle\left\langle\bar{\chi}^{(-)}\left(E_{k}, \Omega\right)|\langle 1 v, n|V| n\rangle| \bar{\chi}^{(+)}\left(E_{k_{1}}, \Omega_{1}\right)\right\rangle \\
& \times a_{1 \mu}^{\mathrm{n}} \overline{E-E_{n}-E_{k_{1}}+\mathrm{i} \varepsilon}\left\langle\bar{\chi}^{(-)}\left(E_{k_{1}}, \Omega_{1}\right)|\langle 1 \mu|V| 0\rangle| \bar{\chi}^{(+)}\left(E_{k_{0}}, \Omega_{0}\right)\right\rangle \delta\left(E_{f}-U\right) .
\end{aligned}
$$

Next, we invoke the leading-particle statistics assumption that $V$ generates random matrix elements so that, upon energy averaging, only the diagonal elements survive. First, we perform this in combination with an average over the final energy. Then, the cross terms of $v, v^{\prime}$ and $n, n^{\prime}$ disappear. We obtain

$$
\begin{aligned}
\frac{\mathrm{d}^{2} \sigma^{(2)}}{\mathrm{d} \Omega \mathrm{d} E_{k}}= & \frac{2 m^{5}}{(2 \pi)^{8} \hbar^{10}} \frac{k}{k_{0}} \sum_{n} \sum_{v} \sum_{\mu} \sum_{\mu^{\prime}} \int \mathrm{d} \Omega_{1}^{\prime} \int \mathrm{d} \Omega_{1} \int \mathrm{d} E_{k_{1}^{\prime}} E_{k_{1}^{\prime}}^{1 / 2} \int \mathrm{d} E_{k_{1}} E_{k_{1}}^{1 / 2} \\
& \times \hat{\rho}_{(1, y) n}(U)\left\langle\bar{\chi}^{(+)}\left(E_{k_{0}}, \Omega_{0}\right)\left|\left\langle 0|V| 1 \mu^{\prime}\right\rangle\right| \bar{\chi}^{(-)}\left(E_{k_{1}^{\prime}}, \Omega_{1}^{\prime}\right)\right\rangle a_{1 \mu}^{n} \frac{1}{E-E_{n}-E_{k_{i}}-\mathrm{i} \varepsilon} \\
& \times \frac{\left\langle\bar{\chi}^{(+)}\left(E_{k_{1}^{\prime}}, \Omega_{1}^{\prime}\right)|\langle n|V| 1 p 1 h, n\rangle| \bar{\chi}^{(-)}\left(E_{k}, \Omega\right)\right\rangle\left\langle\bar{\chi}^{(-)}\left(E_{k}, \Omega\right)|\langle 1 p 1 h, n|V| n\rangle| \bar{\chi}^{(+)}\left(E_{k_{1}}, \Omega_{1}\right)\right\rangle}{} \\
& \times a_{1 \mu}^{n} \frac{1}{E-E_{n}-E_{k_{1}}+\mathrm{i} \varepsilon}\left\langle\bar{\chi}^{(-)}\left(E_{k_{2}}, \Omega_{1}\right)|\langle 1 \mu|V| 0\rangle| \bar{\chi}^{(+)}\left(E_{k_{0}}, \Omega_{0}\right)\right\rangle .
\end{aligned}
$$

This equation can be reduced by noting that the matrix element $\langle 1 v, n|V| n\rangle$ equals the $1 p 1 h$ matrix element $\langle 1 v|V| 0\rangle$ as a consequence of the two-body nature of $V$. Further, a Brink-Axel-like hypothesis can be invoked by replacing the final true partial level density by that of the particlehole pair created in the second step, i.e. $\hat{\rho}_{(1 v) n}(U) \rightarrow \hat{\rho}_{1 v}\left(E_{k_{1}}-E_{k}\right)$, where the argument of $\hat{\rho}_{1 v}$ represents the energy transferred in the second step. This gives

$$
\begin{aligned}
\frac{\mathrm{d}^{2} \sigma^{(2)}}{\mathrm{d} \Omega \mathrm{d} E_{k}}= & \frac{2 m^{5}}{(2 \pi)^{8} \hbar^{10}} \frac{k}{k_{0}} \sum_{n} \sum_{v} \sum_{\mu} \sum_{\mu^{\prime}} \int \mathrm{d} \Omega_{1}^{\prime} \int \mathrm{d} \Omega_{1} \int \mathrm{d} E_{k_{i}^{\prime}} E_{k_{1}^{\prime}}^{1 / 2} \int \mathrm{d} E_{k_{1}} E_{k_{i}}^{1 / 2} \\
& \times \hat{\rho}_{1 v}\left(E_{k_{1}}-E_{k}\right)\left\langle\bar{\chi}^{(+)}\left(E_{k_{0}}, \Omega_{0}\right)\left|\left\langle 0|V| 1 \mu^{\prime}\right\rangle\right| \bar{\chi}^{(-)}\left(E_{k_{1}^{\prime}}, \Omega_{1}^{\prime}\right)\right\rangle a_{1 \mu^{\prime}}^{n} \frac{1}{E-E_{n}-E_{k_{1}^{\prime}}-\mathrm{i} \varepsilon} \\
& \times \frac{\left\langle\bar{\chi}^{(+)}\left(E_{k_{1}^{\prime}}, \Omega_{1}^{\prime}\right)|\langle 0|V| 1 p 1 h\rangle| \bar{\chi}^{(-)}\left(E_{k}, \Omega\right)\right\rangle\left\langle\bar{\chi}^{(-)}\left(E_{k}, \Omega\right)|\langle 1 p 1 h|V| 0\rangle| \bar{\chi}^{(+)}\left(E_{k_{1}}, \Omega_{1}\right)\right\rangle}{} \\
& \times a_{1 \mu}^{n} \frac{1}{E-E_{n}-E_{k_{1}}+\mathrm{i} \varepsilon}\left\langle\bar{\chi}^{(-)}\left(E_{k_{1}}, \Omega_{1}\right)|\langle 1 \mu|V| 0\rangle| \bar{\chi}^{(+)}\left(E_{k_{0}}, \Omega_{0}\right)\right\rangle .
\end{aligned}
$$


Inserting $\delta\left(E_{n}-U^{\prime}\right)$, adding the corresponding integral over $U^{\prime}$ and integrating over $U^{\prime}$ gives

$$
\begin{aligned}
\frac{\mathrm{d}^{2} \sigma^{(2)}}{\mathrm{d} \Omega \mathrm{d} E_{k}}= & \frac{2 m^{5}}{(2 \pi)^{8} \hbar^{10}} \frac{k}{k_{0}} \sum_{n} \sum_{v} \sum_{\mu} \sum_{\mu^{\prime}} \int \mathrm{d} \Omega_{1}^{\prime} \int \mathrm{d} \Omega_{1} \int \mathrm{d} E_{k_{1}^{\prime}} E_{k_{1}^{\prime}}^{1 / 2} \int \mathrm{d} E_{k_{1}} E_{k_{1}}^{1 / 2} \\
& \times \hat{\rho}_{1 v}\left(E_{k_{1}}-E_{k}\right)\left\langle\bar{\chi}^{(+)}\left(E_{k_{0}}, \Omega_{0}\right)\left|\left\langle 0|V| 1 \mu^{\prime}\right\rangle\right| \bar{\chi}^{(-)}\left(E_{k_{1}^{\prime}}, \Omega_{1}^{\prime}\right)\right\rangle a_{1 \mu^{\prime}}^{n} \frac{2 \pi \mathrm{i}}{E_{k_{1}}-E_{k_{1}^{\prime}}+2 \mathrm{i} \varepsilon} \\
& \times \overline{\left\langle\bar{\chi}^{(+)}\left(E_{k_{1}^{\prime}}, \Omega_{1}^{\prime}\right)|\langle 0|V| 1 p 1 h\rangle| \bar{\chi}^{(-)}\left(E_{k}, \Omega\right)\right\rangle\left\langle\bar{\chi}^{(-)}\left(E_{k}, \Omega\right)|\langle 1 p 1 h|V| 0\rangle| \bar{\chi}^{(+)}\left(E_{k_{1}}, \Omega_{1}\right)\right\rangle} \\
& \times a_{1 \mu}^{n}\left\langle\bar{\chi}^{(-)}\left(E_{k_{1}}, \Omega_{1}\right)|\langle 1 \mu|V| 0\rangle| \bar{\chi}^{(+)}\left(E_{k_{0}}, \Omega_{0}\right)\right\rangle \delta\left(E_{n}-\left(E-E_{k_{1}}\right)\right) .
\end{aligned}
$$

The complex fraction in this expression can be written as the sum of a principal part and a delta function part

$$
\frac{\mathrm{i}}{E_{k_{1}}-E_{k_{1}^{\prime}}+2 \mathrm{i} \varepsilon}=\mathrm{i} \mathscr{P}\left(\frac{1}{E_{k_{1}}-E_{k_{1}^{\prime}}}\right)+\pi \delta\left(E_{k_{1}}-E_{k_{1}^{\prime}}\right) .
$$

Upon application of leading-particle statistics to the first step matrix elements, the principal part of Eq. (2.95) and the non-diagonal elements of the sums (integrations) over $\mu, \mu^{\prime}, E_{k_{1}}, E_{k_{1}^{\prime}}, \Omega_{1}, \Omega_{1}^{\prime}$ vanish and we obtain

$$
\begin{aligned}
\frac{\mathrm{d}^{2} \sigma^{(2)}}{\mathrm{d} \Omega \mathrm{d} E_{k}}= & \frac{2 m^{5}}{(2 \pi)^{8} \hbar^{10}} \frac{k}{k_{0}} 2 \pi^{2} \sum_{v} \sum_{\mu} \int \mathrm{d} \Omega_{1} \int \mathrm{d} E_{k_{1}} \hat{\rho}_{1 v}\left(E_{k_{1}}-E_{k}\right) \hat{\rho}_{1 \mu}\left(E_{k_{0}}-E_{k_{1}}\right) \\
& \times \overline{\left.\left\langle\bar{\chi}^{(-)}\left(E_{k}, \Omega\right)|\langle 1 p 1 h|V| 0\rangle| \bar{\chi}^{(+)}\left(E_{k_{1}}, \Omega_{1}\right)\right\rangle\right|^{2}} \\
& \times \overline{\left\langle\left.\left\langle\bar{\chi}^{(-)}\left(E_{k_{1}}, \Omega_{1}\right)|\langle 1 p 1 h|V| 0\rangle| \bar{\chi}^{(+)}\left(E_{k_{0}}, \Omega_{0}\right)\right\rangle\right|^{2}\right.},
\end{aligned}
$$

where we used Eq. (2.83) for the first step. Using Eqs. (2.82) and (2.84), Eq. (2.96) can be rewritten as

$$
\begin{aligned}
\frac{\mathrm{d}^{2} \sigma^{(2)}}{\mathrm{d} \Omega \mathrm{d} E_{k}}= & \frac{m}{4 \pi^{2} \hbar^{2}} \sum_{J_{1}, J_{2}} \int \mathrm{d} \Omega_{1} \int \mathrm{d} E_{k_{1}} E_{k_{1}} \hat{\rho}_{1 p 1 h, J_{2}}\left(E_{k_{1}}-E_{k}\right) \hat{\rho}_{1 p 1 h, J_{1}}\left(E_{k_{0}}-E_{k_{1}}\right) \\
& \times\left[\frac{\mathrm{d} \sigma^{(1)}\left(E_{k} \leftarrow E_{k_{1}}\right)}{\mathrm{d} \Omega}\right]_{J_{2}}^{\mathrm{DWBA}}\left[\frac{\mathrm{d} \sigma^{(1)}\left(E_{k_{1}} \leftarrow E_{k_{0}}\right)}{\mathrm{d} \Omega_{1}}\right]_{J_{1}}^{\mathrm{DWBA}} \\
= & \frac{m}{4 \pi^{2} \hbar^{2}} \int \mathrm{d} \Omega_{1} \int \mathrm{d} E_{k_{1}} E_{k_{1}} \frac{\mathrm{d}^{2} \sigma^{(1)}\left(E_{k}, \Omega \leftarrow E_{k_{1}}, \Omega_{1}\right)}{\mathrm{d} \Omega \mathrm{d} E_{k}} \\
& \times \frac{\mathrm{d}^{2} \sigma^{(1)}\left(E_{k_{1}}, \Omega_{1} \leftarrow E_{k_{0}}, \Omega_{0}\right)}{\mathrm{d} \Omega_{1} \mathrm{~d} E_{k_{1}}},
\end{aligned}
$$

which is equal to Eq. (2.12).

The derivation of the higher step cross-sections proceeds in a similar way. Furthermore, the cross terms of Eq. (2.30), i.e. the products of different order processes, cancel upon energy averaging and the application of leading-particle statistics.

In total, we obtain the FKK cross-section for (multistep) direct processes

$$
\frac{\mathrm{d}^{2} \sigma\left(E_{k}, \Omega \leftarrow E_{k_{0}}, \Omega_{0}\right)}{\mathrm{d} \Omega \mathrm{d} E_{k}}=\sum_{n=1}^{\infty} \frac{\mathrm{d}^{2} \sigma^{(n)}\left(E_{k}, \Omega \leftarrow E_{k_{0}}, \Omega_{0}\right)}{\mathrm{d} \Omega \mathrm{d} E_{k}},
$$


where the first step cross-section reads

$$
\frac{\mathrm{d}^{2} \sigma^{(1)}\left(E_{k}, \Omega \leftarrow E_{k_{0}}, \Omega_{0}\right)}{\mathrm{d} \Omega \mathrm{d} E_{k}}=\sum_{y} \rho_{1 p 1 h . J}\left(E_{k_{0}}-E_{k}\right)\left[\frac{\mathrm{d} \sigma\left(E_{k}, \Omega \leftarrow E_{k_{0}}, \Omega_{0}\right)}{\mathrm{d} \Omega}\right]_{,}^{\mathrm{DWBA}},
$$

and the $n$-step ( $n>1)$ cross-section reads

$$
\begin{aligned}
\frac{\mathrm{d}^{2} \sigma^{(n)}\left(E_{k}, \Omega \leftarrow E_{k_{0}}, \Omega_{0}\right)}{\mathrm{d} \Omega \mathrm{d} E_{k}}= & \frac{m}{4 \pi^{2} \hbar^{2}} \int \mathrm{d} \Omega_{n-1} \int \mathrm{d} E_{k_{n-1}} E_{k_{n-1}} \\
& \times \frac{d^{2} \sigma^{(1)}\left(E_{k}, \Omega \leftarrow E_{k_{n-1}}, \Omega_{n-1}\right)}{\mathrm{d} \Omega \mathrm{d} E_{k}} \frac{\mathrm{d}^{2} \sigma^{(n-1)}\left(E_{k_{n-1}}, \Omega_{n-1} \leftarrow E_{k_{0}}, \Omega_{0}\right.}{\mathrm{d} \Omega_{n-1}} \mathrm{~d} E_{k_{n-1}}
\end{aligned}
$$

The original derivation given by Feshbach et al. (1980) may give the impression that the on-shell approximation [i.e. neglecting the principal part of Eq. (2.95)] is an independent additional assumption. However, the present derivation shows that this is not the case since it is simply a consequence of using the statistics (cf. also Koning and Akkermans, 1991; Feshbach, 1992). Feshbach et al. (1980) take the position that all the nuclear interactions that take place in the multistep process are of a random nature, i.e. leading-particle statistics and residual-system statistics are only two different manifestations of the same physical phenomenon. Mathematically, this is revealed in the paper of Feshbach et al. (1980) by the application of the randomness of the distribution amplitudes to destroy the interference effects of the nuclear states and a subsequent different randomness hypothesis for the non-diagonal terms of the solid angle of the leading particle. In the present derivation, which follows Koning and Akkermans (1991), residual-system statistics [i.e. Eq. (2.80)] is not used.

\subsection{Discussion}

Since the publication of the FKK model in 1980, there has been much debate about its theoretical aspects (and its validity). In this review, it has been our aim to arrange these theoretical aspects in a logical order (which is not necessarily chronological), resulting in a derivation with all physical assumptions invoked at the right place.

Upon the division of the multistep reaction process in an unbound $(P)$ and a bound $(Q)$ chain, the multistep direct reaction model of Feshbach et al. (1980) can be envisaged as the extrapolation to the continuum of (multistep) direct reaction theory for discrete states. Starting from distorted wave theory, the two basic ingredients required to obtain the convolution-type FKK cross-section, see Eqs. (2.1)-(2.4) or Eqs. (2.14)-(2.16), are (in this order!) energy averaging and statistics.

Originally, the FKK formula was derived using statistical approximations only. After criticism of the treatment of the distorted waves (this is also known as the $\chi-\hat{\chi}$ problem, which has been extensively discussed in Section 2.3), the FKK formula was subsequently justified by Feshbach in 1985. Feshbach argued that an extra energy averaging is required before the application of statistical postulates. Otherwise interference effects, that normally would cancel out in the singlechannel case, are wrongly transferred to the statistical multistep expression, yielding an unphysical enhancement of the multistep terms. In mathematical terms, the extra energy averaging leads to the 


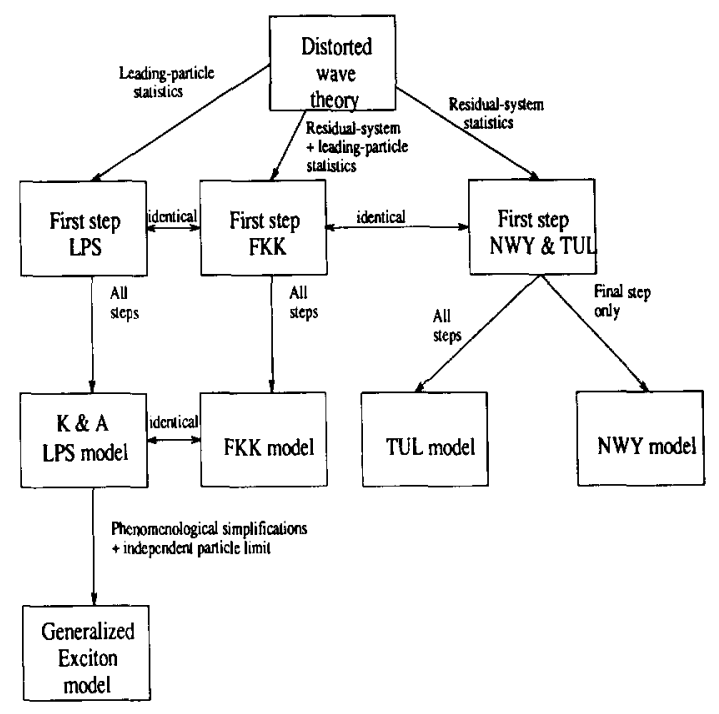

Fig. 8. Family tree of statistical MSD-theories. Here, LPS stands for leading-particle statistics, FKK for Feshbach et al. (1980), TUL for Tamura et al. (1982), NWY for Nishioka et al. (1989) and K \& A for Koning and Akkermans (1993).

replacement of the conjugated outgoing distorted wave $\hat{\chi}^{(+)}$to an energy-averaged incoming distorted wave $\bar{\chi}^{(-)}$. This enables the continuum two-step cross-section to be expressed in terms of two normal DWBA cross-sections.

In the literature on multistep direct reactions, different positions are taken with respect to the statistics. Koning and Akkermans (1991) prove that the application of residual-system statistics alone leads to the multistep direct models of Tamura et al. (1982) and Nishioka et al. (1988), respectively, and that the application of leading-particle statistics alone leads to the FKK model. In other words, for the derivation of the FKK cross-section the randomness of the distribution amplitudes is not required although this randomness was explicitly assumed by Feshbach et al. (1980). Like the energy-averaging procedure described in the previous section, this point has also caused some debate (Feshbach, 1992). Feshbach et al. (1980) take the position that "everything" is random, i.e. there is no physical reason to discriminate between leading-particle statistics and residual-system statistics, although they are seen as separate elements in the mathematical formulation.

However, some authors also make a physical distinction between leading-particle statistics and residual-system statistics. In the models of Tamura et al. (1982) and Nishioka et al. (1988), only residual-system statistics is invoked. Moreover, in the latter model, leading-particle statistics is explicitly forbidden because of the short time scales at which the interaction processes are assumed to take place. Because of the absence of leading-particle statistics in these two models, the off-shell effects of the two-step amplitude still occur in the two-step cross sections. The resulting expressions for the multistep cross section are more complicated than that of the FKK model. Hence, leading-particle statistics is the really critical step for the collapse of the two-step term into two one-step terms (thereby automatically generating the on-shell effect). An intriguing question related to these different viewpoints, leaving aside the mathematical details, is whether one type of statistics can exist without the other. In Fig. 8 the relation between the various multistep direct models is 
depicted in terms of a family tree [which is a revised version of the one given by Koning and Akkermans (1991)]. These models have been categorized according to the different types of statistical assumptions.

Finally, we address the relation of the FKK multistep direct model with semi-classical approaches, in particular the generalized exciton model (Mantzouranis et al., 1976; Akkermans et al., 1980; Costa et al., 1983; Iwamoto and Harada, 1984; for reviews see Gruppelaar et al., 1986; Gadioli and Hodgson, 1992). In the exciton model, it is also assumed that many different particle--hole configurations can be attained from a given one if the system goes to more complex stages. Hence, it basically derives from leading-particle statistics. Residual-system statistics is completely neglected (in fact, it cannot be physically expressed in semi-classical terms). Furthermore, the on-shell approximation is employed as a postulate. The generalized exciton model also displays a convolution-type structure for the double-differential cross-section, the only peculiar feature being that, unlike the FKK model, this only holds for the angle dependency and not for the energy-dependent part. The FKK model can be viewed as the quantum-mechanical variant of the generalized exciton model (Akkermans and Koning, 1990). The fact that it is derived from first quantum-mechanical principles and is conceptually appealing (due to its convolution structure) has resulted in its popularity for practical calculations.

\section{Analyses of experimental data}

\subsection{The general method}

The formulae derived in the previous sections show that a sizeable part of the MSD calculation is the evaluation of the DWBA cross-sections in the continuum for several energies and transferred angular momenta. These are then processed following the MSD prescriptions, giving the required continuum cross-sections.

This method may be understood from Eq. (2.82) for the one-step cross-section, together with Fig. 9. First of all the DWBA cross-sections are computed at a specified excitation energy for each residual spin. Then, after multiplication by the corresponding angular momentum dependent state density and summation over all angular momenta the averaged MSD cross-section per MeV is obtained. The typical forward-peaking remains, whereas the individual oscillatory behaviour of the angular distributions for each residual state averages out, giving a smooth forward-peaked angular distribution, as shown in Fig. 9.

To carry out this calculation, a simple spherical Nilsson model is used to generate the $1 p 1 h$ states. These are grouped in several classes, each class corresponding to the same total angular momentum and region of excitation energy. For a randomly chosen sample of particle-hole states, the DWBA angular distributions are calculated using microscopic two-particle form factors (the hole is treated as a particle). Subsequently, the resulting angular distributions are averaged over the number of states in each class, so that the average DWBA cross-section per $L$-value and per unit of excitation energy is obtained and hence the FKK cross-sections.

In this chapter, the analysis of nucleon reactions below $30 \mathrm{MeV}$, which have both MSC and MSD components, is described in Section 3.2, and of reactions above $30 \mathrm{MeV}$, which are essentially all multistep direct, in Section 3.3. 

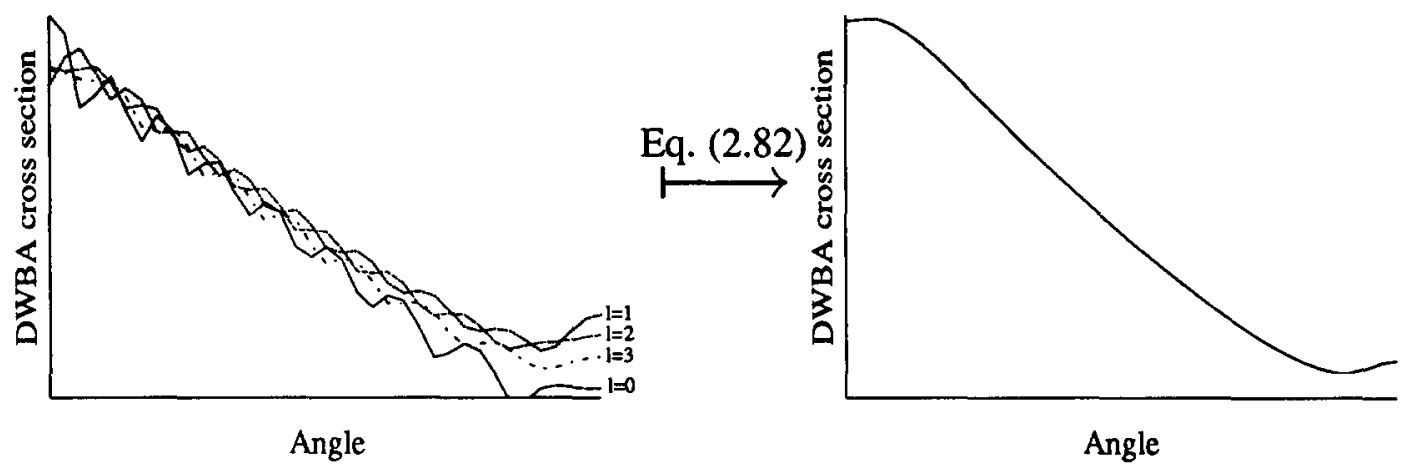

Fig. 9. Smooth forward-peaked angular distribution as a result of the statistical averaging. DWBA cross-sections for transferred angular momenta $0,1,2$ and 3 are calculated for the reaction ( $\left.p, p^{\prime}\right)$ on ${ }^{90} \mathrm{Zr}$ at an incident energy of $80 \mathrm{MeV}$ and an outgoing energy of $60 \mathrm{MeV}$ and subsequently averaged according to Eq. (2.82).

A macroscopic method has recently been developed to perform MSD calculations, and this is described in Section 3.4.

As the incident energy increases, the contribution from the higher steps becomes more important, and since the energy of the incident particle decreases down the chain it becomes possible for transitions from the $P$-chain to the $Q$-chain to take place. The evidence for such processes, together with their effect on the cross-sections, is considered in Section 3.5. The FKK MSD theory has also been applied to alpha-particle reactions, and these are discussed in Section 3.6.

\subsection{Nucleon reactions below $30 \mathrm{MeV}$}

As the incident energy increases, the proportion of MSC processes decreases and that of MSD processes increases. Even at the lower energies, around 10-20 MeV where the MSC process dominates, there is a substantial contribution from MSD processes, and these are considered in Section 3.2.1. At intermediate energies, from 20 to $30 \mathrm{MeV}$, the situation is reversed and MSD dominates, although MSC is not entirely negligible. Such reactions are discussed in Section 3.2.2. At energies above $30 \mathrm{MeV}$ the flux entering the $Q$-chain from the incident channel is negligible, and such reactions are considered in Section 3.3.

\subsubsection{Reactions from 10 to $20 \mathrm{MeV}$}

Multistep direct reactions at lower energies are most marked for the heavier target nuclei, and an example is provided by the analysis of the inelastic scattering of $11.5 \mathrm{MeV}$ neutrons by ${ }^{184} \mathrm{~W}$ made by Marcinkowski et al. (1989). It was found that both the multistep compound and the multistep direct processes contribute substantially to the cross-sections. In the multistep direct calculations, the individual angular distributions for the excitation of sets of particle-hole pairs did not show the striking similarity apparent in Fig. 9. The average distorted wave differential cross-section thus depends rather strongly on the outgoing neutron energy and also quite significantly on the number of contributing particle-hole pairs. To average out these fluctuations it was found necessary to include up to 12 particle-hole states for each $L$-value. This implies averaging the calculated 


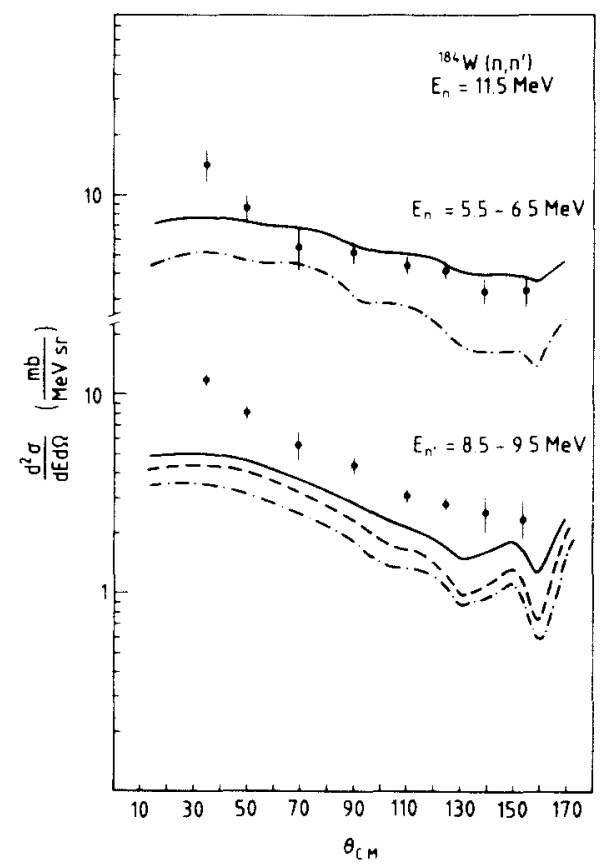

Fig. 10. Double differential cross-sections for the inelastic scattering of $11 \mathrm{MeV}$ neutrons by ${ }^{184} \mathrm{~W}$ compared with the results of one-step direct (dash-dotted curves), plus collective enhancement (dashed curve) plus multistep compound (solid curves) (Marcinkowski et al., 1989).

cross-sections for the individual particle-hole states over a series of overlapping energy intervals with widths up to $9 \mathrm{MeV}$. This averaging interval is comparable with the widths of single-particle states measured by (p, 2p) reactions (Jacob and Maris, 1973). The value of the effective interaction strength used in these calculations was $V_{0}=25 \mathrm{MeV}$.

The results of these calculations are compared with the experimental data in Fig. 10 for two outgoing neutron energies. The angular distributions of the neutrons with lower outgoing energies are quite well reproduced by the sum of the one-step and two-step multistep direct and the multistep compound calculations.

For lighter target nuclei, the double differential cross-sections for neutron inelastic scattering at $14 \mathrm{MeV}$ are nearly symmetric about $90^{\circ}$, indicating predominantly MSC and compound nucleus processes, but in addition there is a forward excess attributable to MSD processes.

This MSD contribution is most clearly shown by plotting the difference between the double differential cross-sections at two complementary angles in the centre of mass. This removes the MSC and the compound nucleus contributions since they are symmetric about $90^{\circ}$, leaving only the MSD, together with some direct collective excitation cross-section at the higher emission energies. Some results for ${ }^{93} \mathrm{Nb}\left(\mathrm{n}, \mathrm{n}^{\prime}\right)$ at $14 \mathrm{MeV}$ are shown in Fig. 11 compared with similarly subtracted MSD calculations. For each pair of angles the MSD fits well, apart from the higher emission energies where the collective excitation is prominent, and the mean value of $V_{0}$ is $33 \mathrm{MeV}$. The MSD cross-section found in this way is in good agreement with that found by Chadwick and Young (1993) using a somewhat different method. 


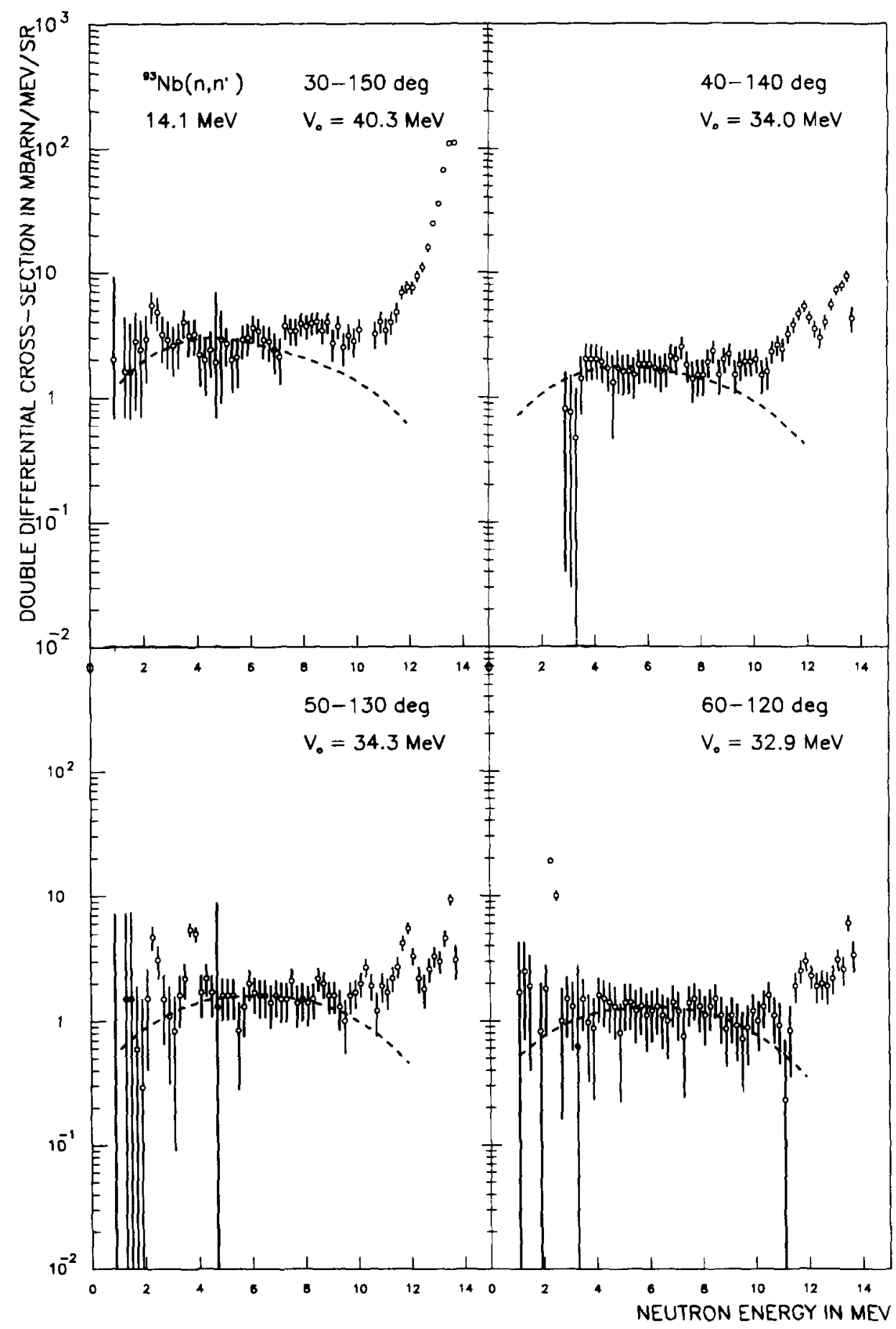

Fig. 11. Subtracted double differential cross-sections in the CM system for $14 \mathrm{MeV}$ neutrons inelastically scattered by ${ }^{93} \mathrm{Nb}$ for four pairs of complementary angles. The curves are similarly subtracted cross-sections calculated with the multistep direct theory of Feshbach et al. (1980). The peaks at higher outgoing energies are due to the excitation of collective states, a process not included in the calculations. The curves are normalised to the data, giving the values of the effective interaction strength $V_{0}$ given in each box. The experimental points are obtained from the data of Takahashi et al. (1992) (Demetriou et al. 1993). 


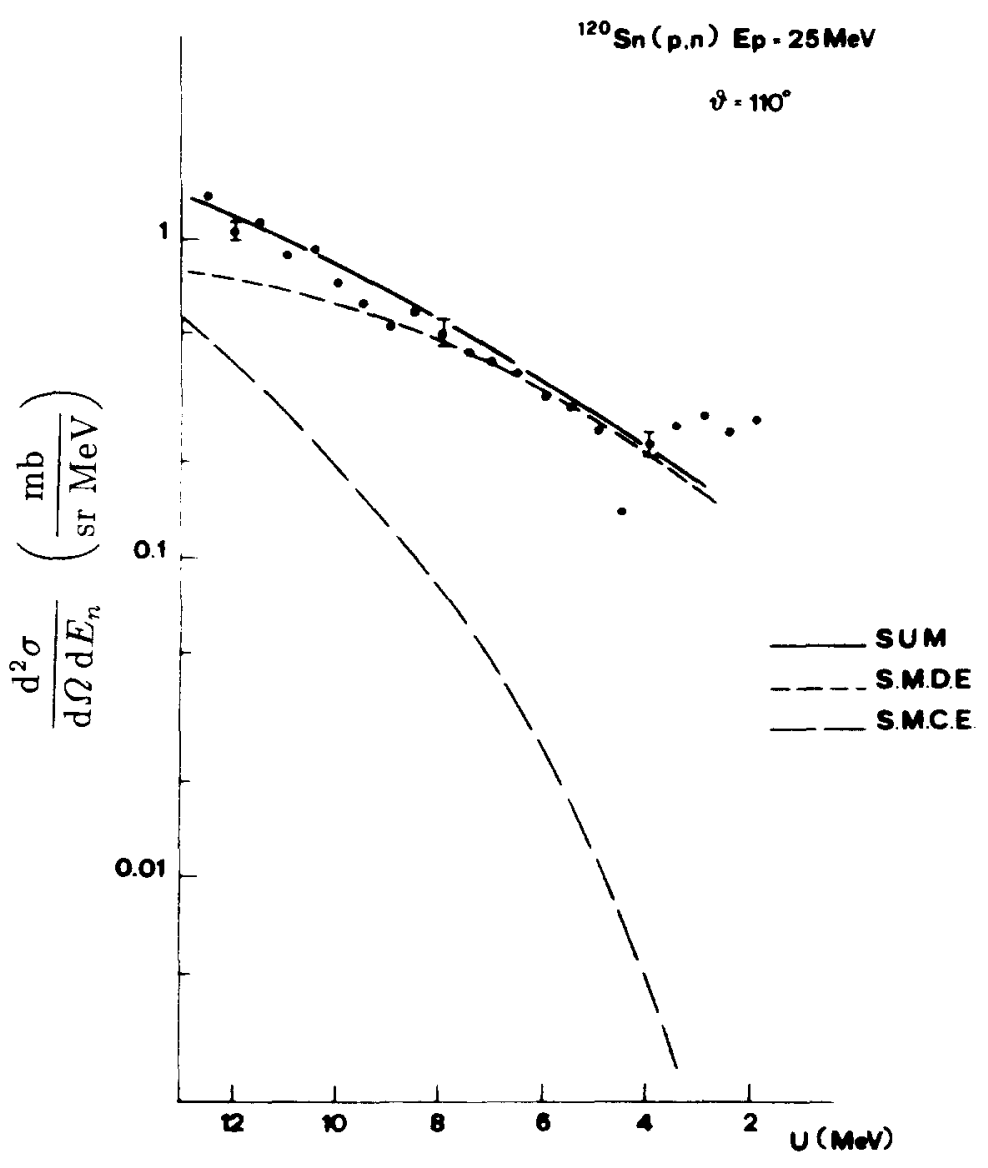

Fig. 12. Energy spectra at $110^{\circ}$ for the ${ }^{120} \mathrm{Sn}(\mathrm{p}, \mathrm{n})$ reaction at $25 \mathrm{MeV}$ compared with MSC (long-dash curve) and MSD (short-dash curve) calculations. The full curve is the sum of these processes (Bonetti and Colli-Milazzo, 1984).

\subsubsection{Reactions from 20 to $30 \mathrm{MeV}$}

The first analysis of data at intermediate energies including both MSC and MSD processes was made by Bonetti and Colli-Milazzo (1984) for the ${ }^{120} \mathrm{Sn}(\mathrm{p}, \mathrm{n})$ reaction at $25 \mathrm{MeV}$. Their results for the neutron spectrum at $110^{\circ}$ and for the angular distribution at residual nucleus energies of 8 and $12 \mathrm{MeV}$ are shown in Figs. 12 and 13. It is found that most of the cross-section is due to the MSD process, but that particularly at backward angles and higher residual nucleus energies (corresponding to lower outgoing neutron energies) the contribution from MSC processes is also important. It is notable that both MSC and MSD calculations were made with the same value of the effective nucleon-nucleon interaction strength $V_{0}=25 \mathrm{MeV}$.

The double differential cross-section for the ${ }^{65} \mathrm{Cu}(\mathrm{p}, x \mathrm{n})$ reaction at $26.7 \mathrm{MeV}$ has been compared with the semi-classical and quantum mechanical pre-equilibrium theories by Holler et al. (1985). As shown in Fig. 14(a), the geometry-dependent hybrid model is able to describe quite well the energy dependence of the high energy emitted particles but does not give accurately the observed intensity of emitted particles in the most backward direction. The sum of the multistep 


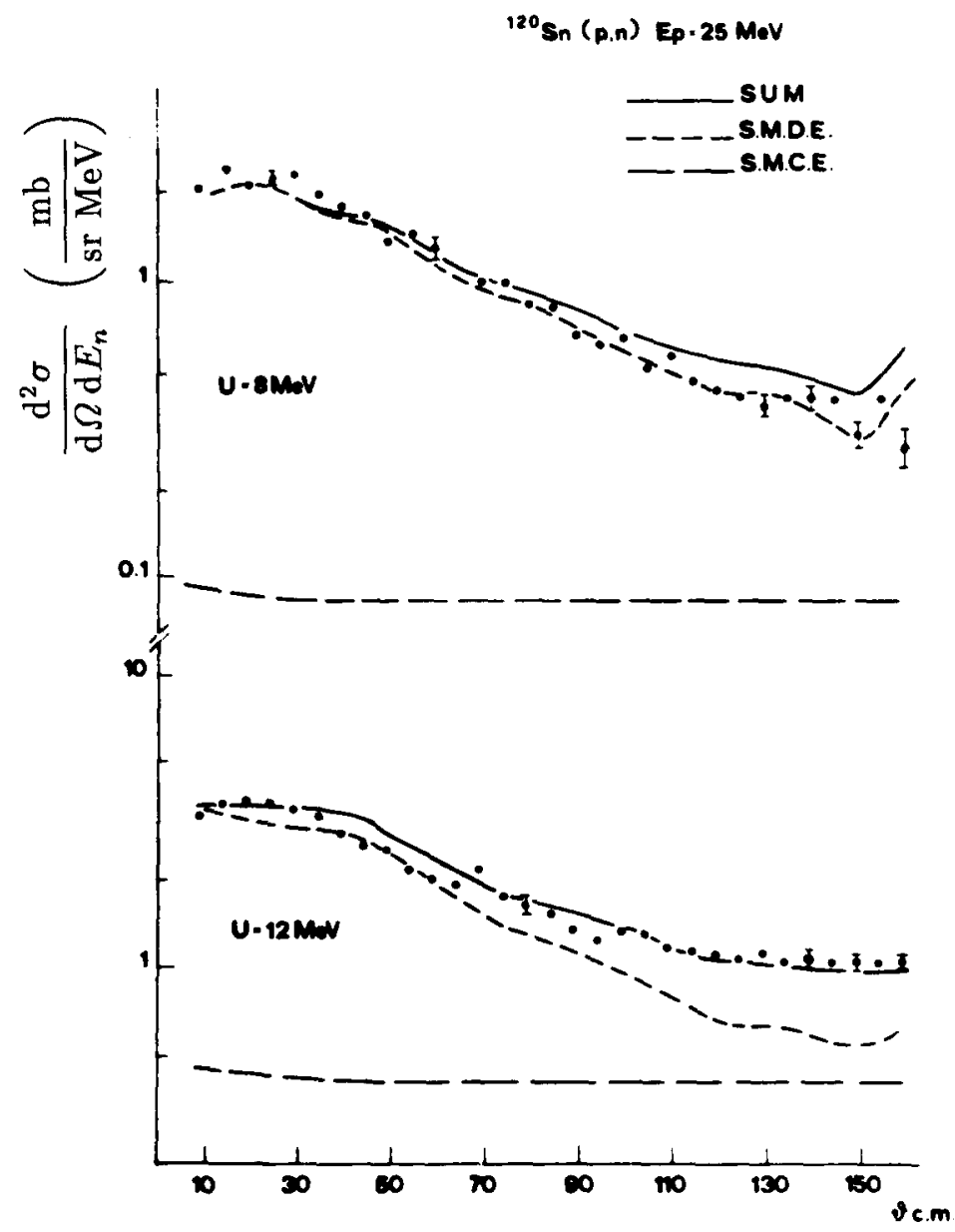

Fig. 13. Angular distributions at $U=8 \mathrm{MeV}$ and $U=12 \mathrm{MeV}$ for the ${ }^{120} \mathrm{Sn}(\mathrm{p}, \mathrm{n})$ reaction at $25 \mathrm{MeV}$ compared with MSC (long-dash curve) and MSD (short-dash curve) calculations (Bonetti and Colli-Milazzo, 1984).

compound and multistep direct pre-equilibrium cross-sections evaluated with the FKK theory gave cross-sections in good accord with the data, as shown in Fig. 14(b). It is notable that the same value $V_{0}=27 \mathrm{MeV}$ was used for both multistep direct calculations. At this energy most of the cross-section at the higher emission energies is multistep direct.

The effects of pairing on pre-equilibrium emission were studied by Mordhorst et al. (1986) who measured the outgoing neutron spectrum from the $(p, x n)$ reaction at $25.6 \mathrm{MeV}$ on seven molybdenum isotopes. At this energy both multistep direct and multistep compound processes contribute substantially, and the experimental data were fitted with the same value of $V_{0}=25 \mathrm{MeV}$ using a Yukawa effective interaction of range $1 \mathrm{fm}$. The cross-sections show a strong even-odd dependence due to the different pairing energies.

The Feshbach, Kerman and Koonin theory has been applied by Marcinkowski et al. (1989) to analyse the inelastic scattering of neutrons by ${ }^{184} \mathrm{~W}$, as described in the previous section. In 

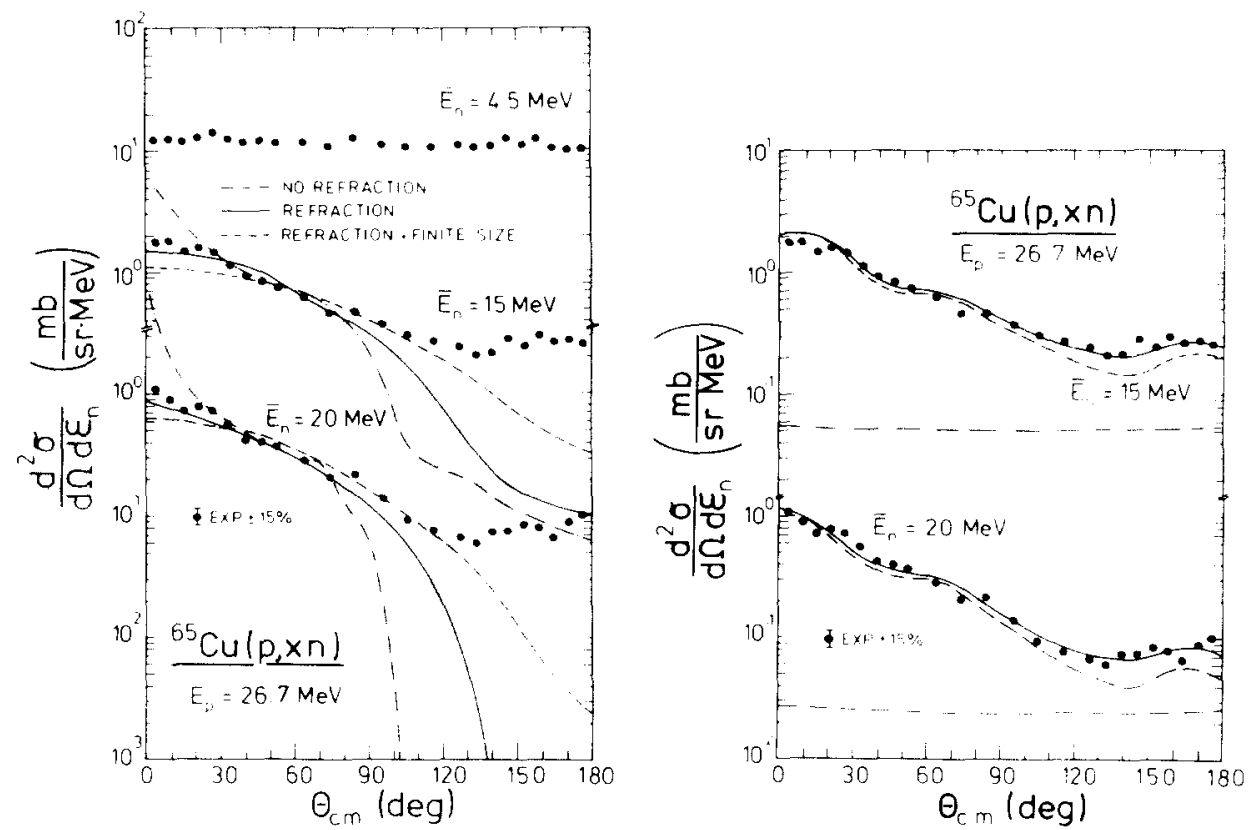

Fig. 14. (a) Angular distributions for the ${ }^{65} \mathrm{Cu}(\mathrm{p}, \mathrm{xn})$ reaction at $26.7 \mathrm{MeV}$ compared with geometry-dependent hybrid model calculations. The data are plotted in $1 \mathrm{MeV}$ bins centred at energies $E$. The calculations are shown for pure NN-scattering (dot-dash), NN-scattering with entrance channel refraction (solid) and with refraction plus finite size correction (dashed). (b) Angular distributions for the ${ }^{65} \mathrm{Cu}(\mathrm{p}, \mathrm{xn})$ reaction at $26.7 \mathrm{MeV}$ compared with FKK quantum-mechanical calculations. The statistical multistep direct contributions are shown by the dashed line, the statistical multistep compound by the dash-dot line and their sum by the solid line (Holler et al., 1985).

addition to the data for $11 \mathrm{MeV}$ neutrons already mentioned, they also analysed data for $26 \mathrm{MeV}$ neutrons, and their results are shown in Fig. 15. At the highest outgoing energies the experimental cross-sections are greater than the calculated ones, and this is attributable to particular direct processes such as the excitation of low-lying quadrupole and octupole surface vibrations (Marcinkowski et al., 1983b; Kalka et al., 1988). The contributions of such processes were estimated using the energy-weighted sum rule for isoscalar transitions, and is also shown in the figure. Comparison with the experimental cross-sections shows that there is still some cross-section unaccounted for at these higher outgoing energies.

The ${ }^{93} \mathrm{Nb}\left(\mathrm{n}, \mathrm{n}^{\prime}\right)$ reaction at $25.7 \mathrm{MeV}$ has been analysed using many semi-classical pre-equilibrium models which generally fit the data well (Gruppelaar et al., 1986; Gruppelaar and Nagel, 1985). Fig. 16 shows the quantum-mechanical MSD energy spectrum calculated by the program KAPSIES (Koning and Akkermans, 1993) compared with the experimental data of Marcinkowski et al. (1983). The cross-sections were measured only for outgoing energies above $12 \mathrm{MeV}$, and it is seen that the high energy part of the cross-section is well-described by the MSD process. At low emission energies, the cross-section increases whereas the MSD contribution remains approximately constant. In this region, the cross-section is mainly due to the multistep compound, compound and multiple emission processes. The figure also shows the cross-section calculated with the program GNASH (Arthur, 1988; Young, Arthur and Chadwick, 1992), which gives a reliable fit 


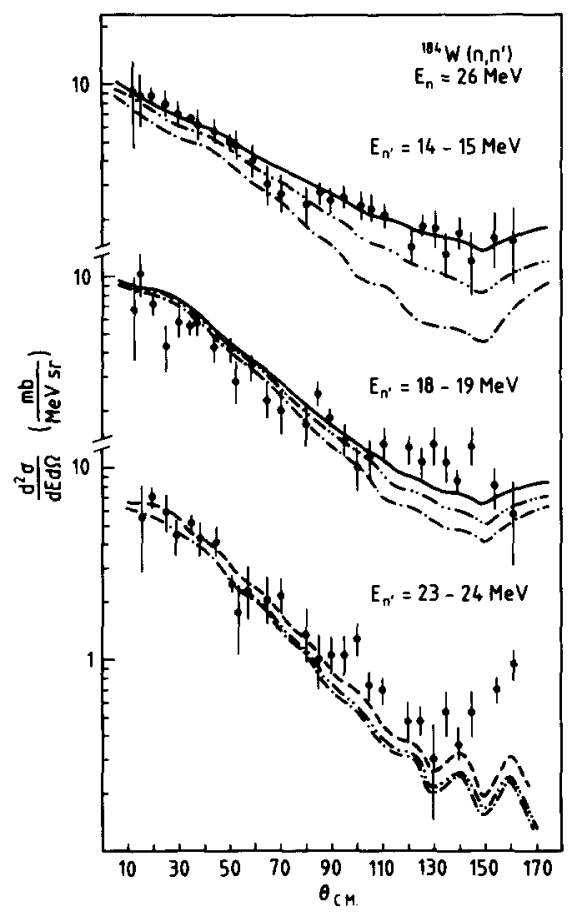

Fig. 15. Double differential cross-section for the inelastic scattering of $28 \mathrm{MeV}$ neutrons by ${ }^{184} \mathrm{~W}$ compared with one-step direct (dash-dotted curves), plus two-step direct (dash-double dotted curves) plus collective contributions (dashed curve) plus multistep compound (solid curves) (Marcinkowski et al. 1989).

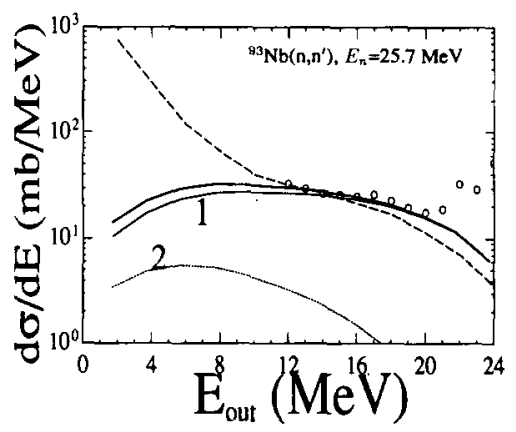

Fig. 16. The outgoing energy spectrum for the ${ }^{93} \mathrm{Nb}\left(n, n^{\prime}\right)$ reaction at $25.7 \mathrm{MeV}$ compared with MSD calculations. The curves labelled 1 and 2 show the contributions of the first and second steps, and the full line their total. The dashed line shows the results of a calculation using the program GNASH, which include the compound nucleus contributions important at low emission energies (Koning and Akkermans, 1993).

for the whole range of outgoing energies (see Fig. 3(D) of Gruppelaar and Nagel, 1985 or Fig. 11 of Gruppelaar et al., 1986). These calculations include the Hauser-Feshbach compound nucleus and the exciton model direct reaction components. These associated angular distributions are given in Fig. 17 and show some oscillatory behaviour at back angles. Study of the individual DWBA 

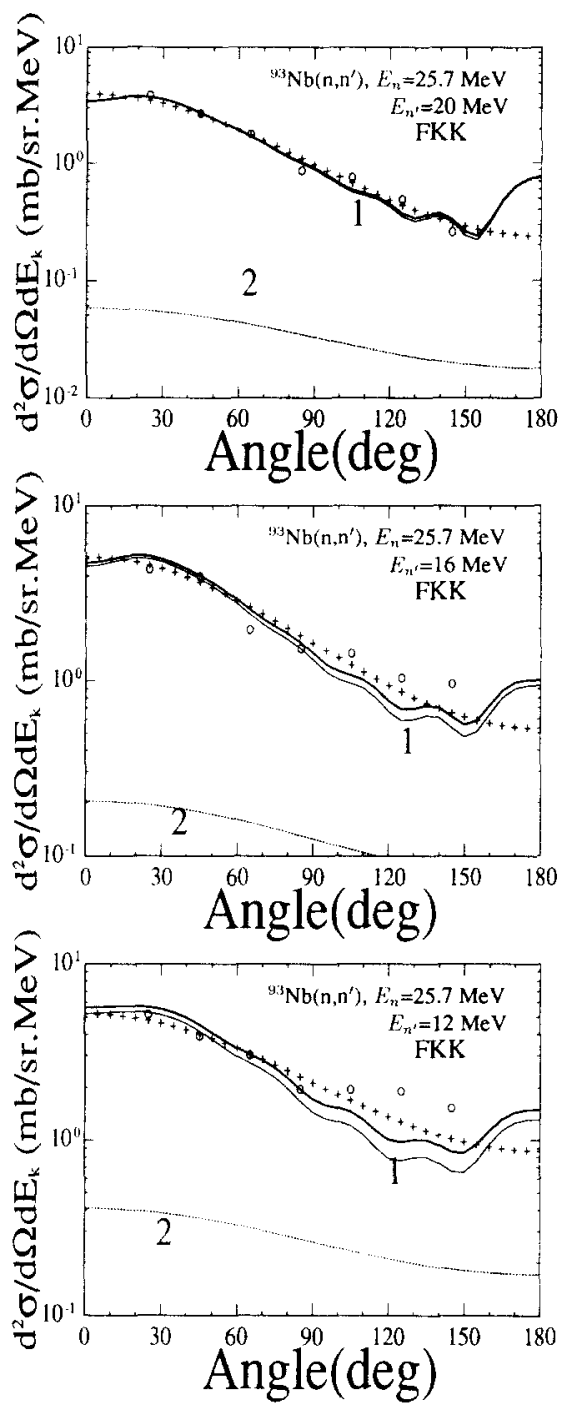

Fig. 17. The angular distribution for the ${ }^{93} \mathrm{Nb}\left(\mathrm{n}, \mathrm{n}^{\prime}\right)$ reaction at $25.7 \mathrm{MeV}$ for three outgoing energies compared with MSD calculations. The curves labelled 1 and 2 show the contributions of the first and second stages, and the full line their total. The pluses show the systematics of Kalbach (Koning and Akkermans, 1993).

components shows that there is some constructive interference which is not washed out by the averaging process, as in Fig. 16. This oscillatory behaviour disappears at higher incident energies.

An independent FKK analysis of the same reaction has been made by Chadwick and Young (1993), and their results are shown in Fig. 18 for the energy spectrum and Fig. 19 for the angular distributions. The results are very similar to those of Figs. 16 and 17, allowing for the absence of MSC and compound nucleus contributions in the work of Koning and Akkermans (1993). These analyses both show that at these relatively low incident energies the one-step direct component is far more important than the multistep direct contributions. 

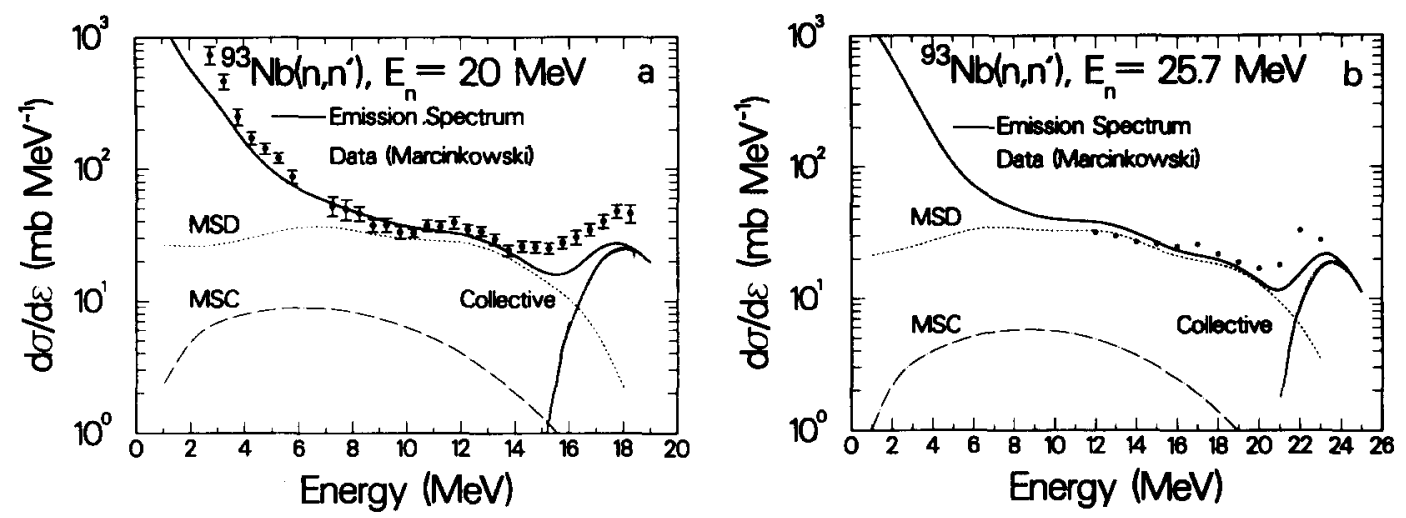

Fig. 18. Angle-integrated energy spectra for the ${ }^{93} \mathrm{Nb}\left(\mathrm{n}, \mathrm{n}^{\prime}\right)$ reaction at 20 and $25.7 \mathrm{MeV}$ (Marcinkowski et al., 1991, 1983) compared with FKK MSD and MSC calculations, supplemented by Hauser-Feshbach calculations using the program FKK-GNASH (Chadwick and Young, 1993).
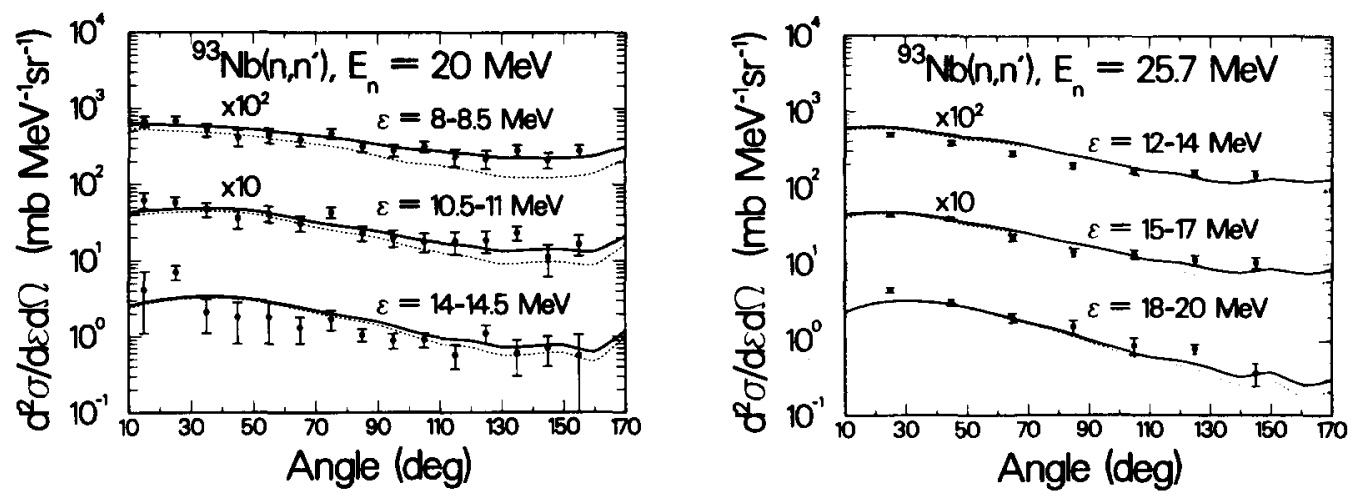

Fig. 19. Angular distributions for the ${ }^{93} \mathrm{Nb}\left(\mathrm{n}, \mathrm{n}^{\prime}\right)$ reaction at 20 and $25.7 \mathrm{MeV}$ (Marcinkowski et al., 1991, 1983) for three outgoing energy bins compared with FKK MSD and MSC calculations, supplemented by Hauser-Feshbach calculations using the program FKK-GNASH. The dashed lines show the MSD alone, and the full lines include the MSC and Hauser-Feshbach contributions (Chadwick and Young, 1993).

\subsection{Reactions above $30 \mathrm{MeV}$}

Bonetti et al. (1981) used the MSD formalism to calculate the double differential cross-sections for $(\mathrm{p}, \mathrm{n})$ reactions on ${ }^{40} \mathrm{Ca},{ }^{90} \mathrm{Zr}$ and ${ }^{208} \mathrm{~Pb}$ at $45 \mathrm{MeV}$ and on ${ }^{120} \mathrm{Sn}$ at 25 and $35 \mathrm{MeV}$. Calculations for the same reaction on ${ }^{120} \mathrm{Sn}$ at $45 \mathrm{MeV}$ have been made by Avaldi et al. (1980). The results for the ${ }^{90} \mathrm{Zn}(\mathrm{p}, \mathrm{n})$ reaction at $45 \mathrm{MeV}$ are compared with the data of Galonsky (1981) in Fig. 20 as a function of angle for several values of the energy of the residual nucleus. It is notable that the theory fits the data well for the lower values of $U$, corresponding to the higher emission energies. There are discrepancies at lower emission energies, probably attributable to the presence of multistep compound reactions, which were not included in the calculations. The comparison 


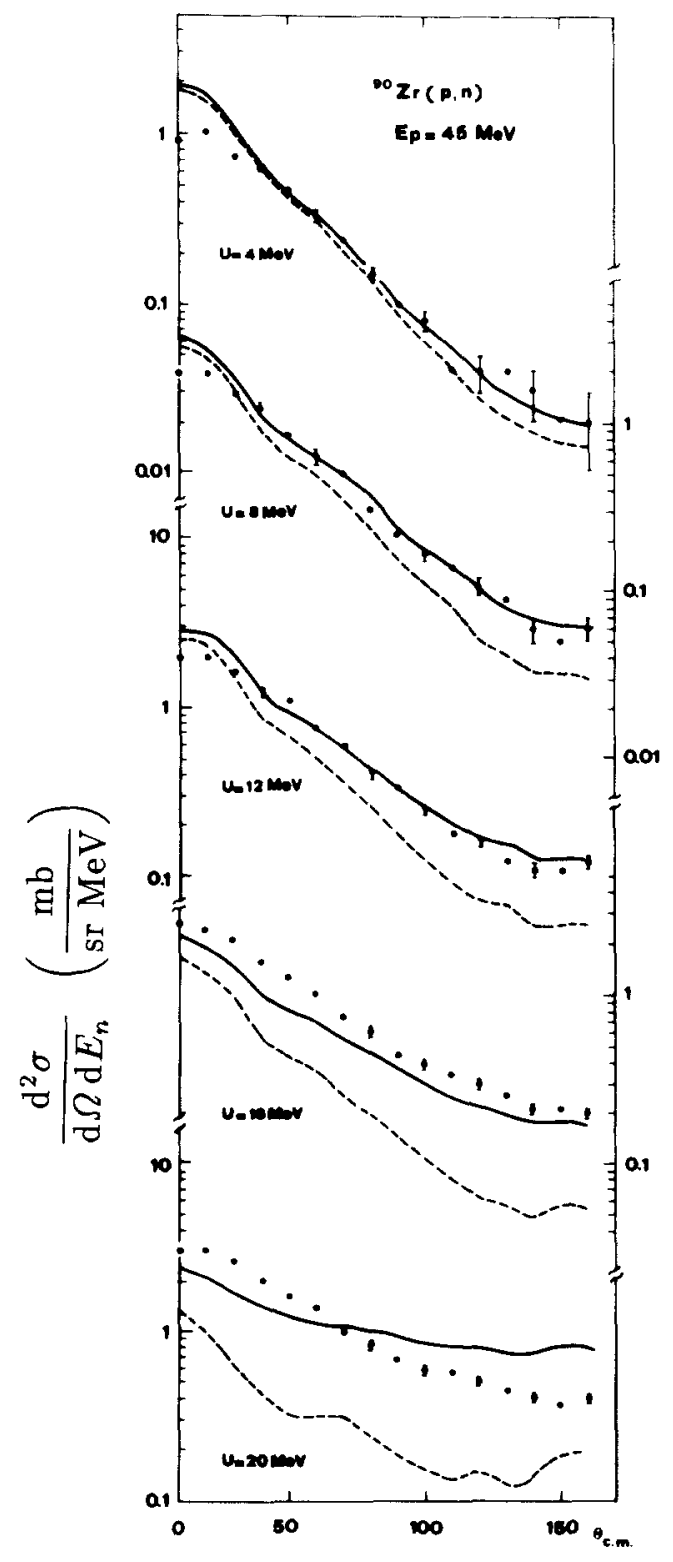

Fig. 20. Double differential cross-section for the ${ }^{90} \mathrm{Zr}(\mathrm{p}, \mathrm{n})$ reaction at $45 \mathrm{MeV}$ compared with multistep direct calculations. The dashed curves show the single-step contributions and the full curves the sum of the single step and multistep contributions (Bonetti et al., 1981).

also shows that more than half of the cross-section comes from the first stage of the reaction. Furthermore, the second and third stage emission is more important at backward angles, as the memory of the direction of the incident particle is progressively lost.

In these calculations the only adjustable parameter is the strength $V_{0}$ of the effective interaction, and all the values required to fit the data for incident energies up to about $50 \mathrm{MeV}$ are consistent 
with the value $V_{0}=27.9 \pm 3.5$ found by Austin (1980) from an analysis of many inelastic scattering reactions to discrete states of the final nucleus. Since the effective interaction enters at each stage of the reaction, the final $m$-stage cross-section is proportional to $V_{0}^{2 m}$ and so the value of $V_{0}$ strongly determines the relative importance of the contributions from the different stages. It is thus more than an overall normalisation coefficient; it also affects the spectral shapes and angular distributions. The overall agreement of the calculated cross-sections with the experimental data as a function of both energy and angle is thus a stringent test of the validity of the theory.

The multistep direct theory has been extended by Bonetti et al. (1982) to give the analysing powers of the emitted particles. Such data are more sensitive to the details of the reaction and thus provide a severe test of any theory proposed to account for them. The analysing power is expected to depend on the stage of the reaction, decreasing down the chain as the memory of the initial polarisation is lost. Measurements of the analysing power of the ${ }^{58} \mathrm{Ni}\left(\mathrm{p}, \mathrm{p}^{\prime}\right)$ reaction by Sakai et al. (1980) showed large and positive analysing powers at backward angles.

The analysing powers may be calculated using the multistep direct formalism, introducing the distinction between the cross-sections for emission to the left and to the right of the incident beam. This was done by appropriate limitations on the integrations over the intermediate angles to the values for each left and right cross-section. It is of course necessary in the evaluation of the distorted wave integrals to include the spin dependence of the effective interaction and this was done by including a complete microscopic model which includes the $L \cdot S$ term. The inclusion of this term was found to be essential in previous calculations of the analysing powers of reactions to discrete nuclear states. The parameters of the calculation were chosen as far as possible from other published analyses, and the final results for the double differential cross-section and analysing powers of the ${ }^{58} \mathrm{Ni}\left(\mathrm{p}, \mathrm{p}^{\prime}\right)$ reaction at $65 \mathrm{MeV}$ are compared with the data of Sakai et al. (1980) in Figs. 21 and 22. The fits to the double differential cross-sections are quite satisfactory, and the overall features of the analysing powers are reproduced quite well. It is particularly notable that it is not possible to reproduce the magnitude and the energy variation of the analysing power in the backward direction by a single step calculation, even including the effect of the microscopic spin-orbit potential. Inclusion of the multistep emission is thus essential to account for the observed analysing powers. The deterioration in the quality of the fits at high excitation energies and thus at low emission energies may be due to the neglect of secondary particle emission, which is more important at these energies.

Comparison of these results with the previous work on $(p, n)$ reactions shows that the relative contributions of the various stages of the reaction are quite similar. It is found, as expected, that the contribution of the first stage in the reaction passes through a minimum as the incident energy increases. At low energies there is insufficient energy for many stages, while at high energies many stages become less likely because of the fall in the value of the interaction matrix with increasing energy. Thus the analyses of $(\mathrm{p}, \mathrm{n})$ reactions gave the contribution of the first stage as about $82 \%$ and $55 \%$ at 25 and $45 \mathrm{MeV}$, respectively, and that of the (p, $\left.\mathrm{p}^{\prime}\right)$ reaction which gave about $70 \%$ at $62 \mathrm{MeV}$. An analysis by Tamura et al. (1977) of the (p, $\left.\mathrm{p}^{\prime}\right)$ reaction gave $90 \%$ at $62 \mathrm{MeV}$.

The multistep direct theory has also been applied by Trabandt et al. $(1988,1989)$ to analyse the neutron emission from the $(\mathrm{p}, x \mathrm{n})$ reaction on ${ }^{90} \mathrm{Zr}$ and ${ }^{208} \mathrm{~Pb}$ at $80 \mathrm{MeV}$. With a Yukawa interaction of range $1 \mathrm{fm}$ and strength $V_{0}=20 \mathrm{MeV}$ they obtained good agreement with the angular distributions of the emitted neutrons at various outgoing energies. Calculations based on the hybrid model of Blann et al. (1984) were able to fit the angle-integrated neutron spectra to 

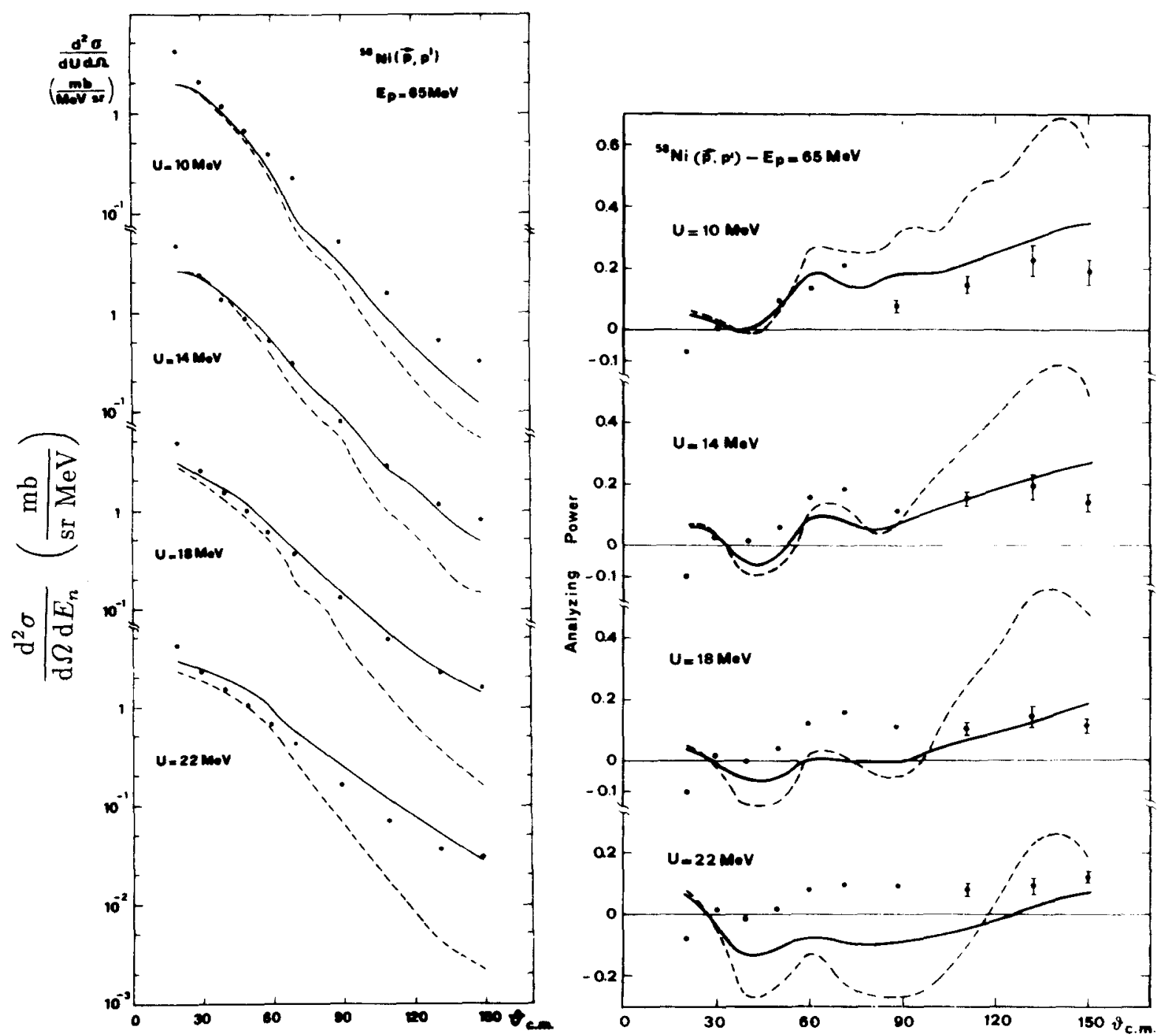

Fig. 21. Double differential cross-section for the ${ }^{58} \mathrm{Ni}\left(\mathrm{p}, \mathrm{p}^{\prime}\right)$ reaction at $65 \mathrm{MeV}$ compared with multistep direct calculations. The dashed curves show the single-step contribution and the full curves the sum of the single and multistep contributions. It is notable that the multistep contribution increases with increasing excitation energy and scattering angle (Bonetti et al., 1982).

Fig. 22. Analysing power for the ${ }^{58} \mathrm{Ni}\left(\mathrm{p}, \mathrm{p}^{\prime}\right)$ reaction at $65 \mathrm{MeV}$ compared with multistep direct calculations. The dashed curves shows the single-step contribution and the full curves the sums of the single and multistep contributions (Bonetti et al., 1982).

within a factor of two overall, but not the angular distributions at various outgoing energies, particularly in the backward direction. The value of the effective interaction strength at this energy is less than the values found at lower energies previously discussed, indicating that it decreases with increasing incident energy. 
Table 1

Values of the effective strength $V_{0}$ of the Yukawa potential of range $1 \mathrm{fm}$ for nucleon interactions

\begin{tabular}{llll}
\hline Reference & Reaction & Energy & $V_{0}(\mathrm{MeV})$ \\
\hline Marcinkowski et al. (1989) & $\left(\mathrm{n}, \mathrm{n}^{\prime}\right)$ & 11.26 & 25 \\
Demetriou et al. (1993) & $\left(\mathrm{n}, \mathrm{n}^{\prime}\right)$ & 14 & 33 \\
Austin (1980) & $\left(\mathrm{N}, \mathrm{N}^{\prime}\right)$ & $35 \pm 15$ & 27.9 \\
Holler et al. (1985) & $(\mathrm{p}, \mathrm{n})$ & 26.7 & 27 \\
Mordhorst et al. (1986) & $(\mathrm{p}, \mathrm{n})$ & 25.6 & 25 \\
Trabandt et al. (1988, 1989) & $(\mathrm{p}, \mathrm{n})$ & 80 & $20 \pm 1$ \\
Cowley et al. (1991) & $\left(\mathrm{p}, \mathrm{p}^{\prime}\right)$ & 80 & $23 \pm 1$ \\
Scobel et al. (1990) & $(\mathrm{p}, \mathrm{n})$ & 120 & $16 \pm 1$ \\
& $\left(\mathrm{p}, \mathrm{p}^{\prime}\right)$ & $($ See Table 2) & $12.5 \pm 1$ \\
Richter et al. (1992,1994) & $(\mathrm{p}, \mathrm{n})$ & 256 & $8.5 \pm 1.5$ \\
Stamer et al. (1993) & & & \\
\hline
\end{tabular}

Subsequently, this analysis was extended to $(\mathrm{p}, \mathrm{n})$ reactions at 120 and $160 \mathrm{MeV}$, and the effective interaction strength was again found to decrease with increasing energy (see Table 1) (Scobel et al., 1990; Stamer et al., 1993). Further measurements of the double differential inelastic scattering to the continuum have been made by Cowley et al. (1991) for 80 and $120 \mathrm{MeV}$ protons on ${ }^{90} \mathrm{Zr}$ and by Richter et al. (1992) from 100 to $200 \mathrm{MeV}$ on ${ }^{58} \mathrm{Ni},{ }^{100} \mathrm{Mo}$ and ${ }^{197} \mathrm{Au}$. They analysed their data with the Feshbach-Kerman-Koonin multistep direct theory and found good agreement with the experimental data as shown in Fig. 23. The contributions of the successive stages to the crosssections at outgoing energies of 20 and $60 \mathrm{MeV}$ for an incident energy of $80 \mathrm{MeV}$ are shown in Fig. 24. The contributions of the first stage agree well with the semi-classical distorted wave calculations of Luo and Kawai (1991).

In all these analyses of multistep direct and multistep compound reactions it is usual to treat the effective interaction strength $V_{0}$ as an adjustable parameter and to fix it by fitting the absolute value of the differential cross-section. It is then important to examine the values obtained and see whether they behave in a systematic way that can be connected with other types of analysis. This comparison is, however, complicated by the progressive improvement in the formulation of the theory, and in the computer programs since each improvement affects the value of $V_{0}$ obtained. Here we consider only the calculations with realistic wavefunctions and a Yukawa two-body interaction of range $1 \mathrm{fm}$.

The results of several analyses are shown in Table 1 . The spread of values of $V_{0}$ is due partly to other differences in the analyses and also to the energy dependence of the effective interaction. Thus the multistep direct analysis of Bonetti et al. (1982) was made with a preliminary version of the program with $V_{0}=25 \mathrm{MeV}$ for the first step and $V_{0}=15 \mathrm{MeV}$ for subsequent steps, so these values are not included in the table. Some analyses were made distinguishing between neutrons and protons in the intra-nuclear cascade; this has rather little effect (less than 10\%) on the shape of the cross-section but requires an increased $V_{0}$ in the case of the more recent analyses of $(\mathrm{p}, \mathrm{n})$ reactions.

The values of $V_{0}$ are found to decrease with increasing incident energy and this is indeed what would be expected from the similar decrease of the real optical potential, which also depends on the strength of the two-body interaction. We can thus estimate the energy variation of $V_{0}$ by assuming 


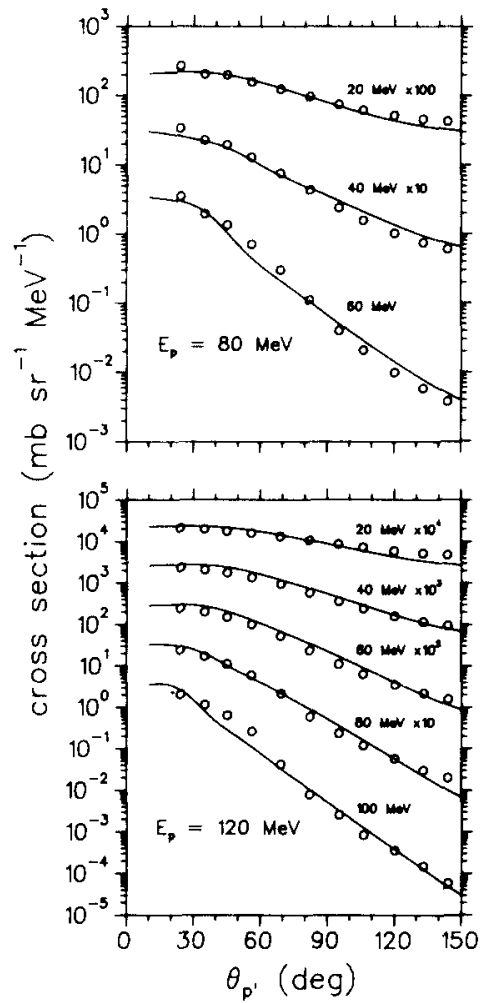

Fig. 23. Double differential cross-section for the inelastic scattering of 80 and $120 \mathrm{MeV}$ protons by ${ }^{90} \mathrm{Zr}$ at several outgoing energies compared with calculations using the Feshbach-Kerman-Koonin multistep direct theory (Cowley et al., 1991).

that it has the same energy variation as the real optical potential and normalising to the average of several determinations at lower energies. We use for this purpose the value $V_{0}=27.9 \pm 3.5 \mathrm{MeV}$ obtained by Austin (1980) from a survey of the analyses of proton scattering at around 20 to $50 \mathrm{MeV}$ to discrete final states, and also those found by Holler et al. (1985) and by Marcinkowski et al. (1989).

Since the incident particle loses energy as it passes from stage to stage in the multistep process it would be more exact to allow the effective interaction to increase down the chain. It is, however, simpler to use an averaged value, but this effect should be taken into account when comparing the energy dependence of $V_{0}$ with that of the optical potential.

An estimate of the magnitude of the effect can be made by assuming that the incident particle loses about half its energy in the first interaction, and emission from the first and second stages are equally likely (Scobel et al., 1990). This would reduce the energy-dependent term by about a factor of $\frac{3}{4}$. The real optical potential, normalised to the average low energy value of $V_{0}=28 \mathrm{MeV}$ at $20 \mathrm{MeV}$ is

$$
V_{0} \approx 32-0.2 E
$$



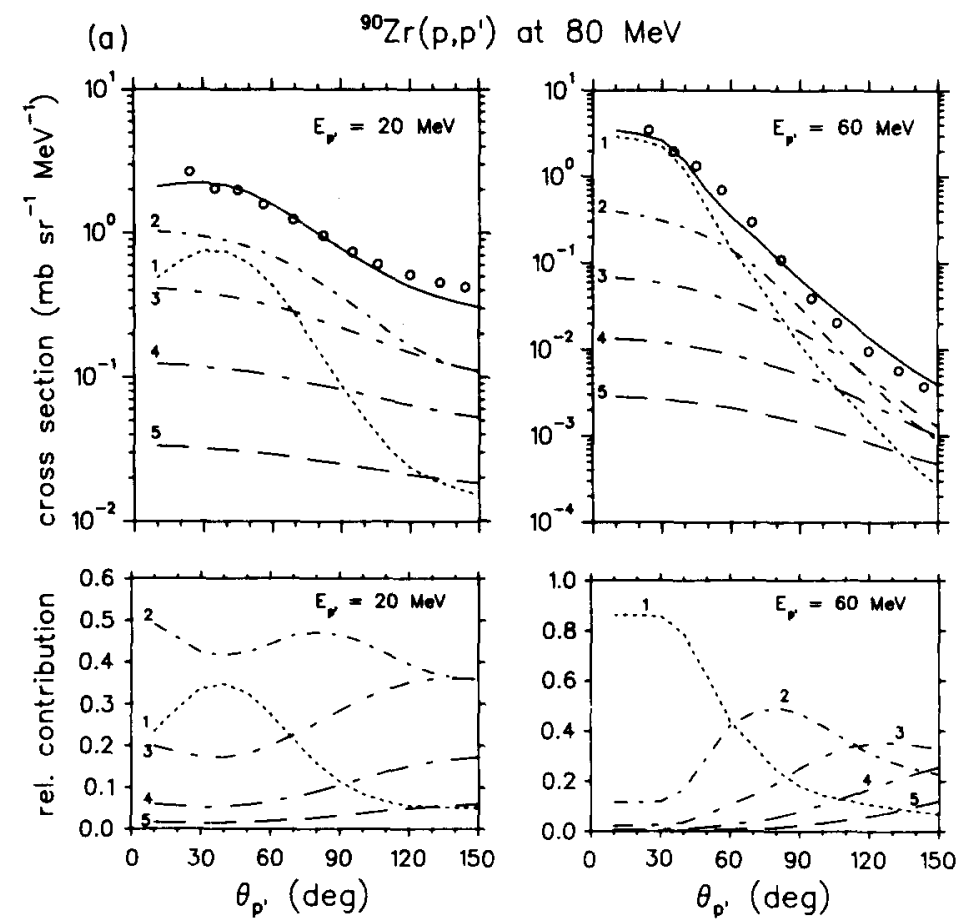

Fig. 24. Double differential cross-section for the inelastic scattering of protons by ${ }^{90} \mathrm{Zr}$ at $80 \mathrm{MeV}$ for outgoing proton energies of 20 and $60 \mathrm{MeV}$ compared with multistep direct calculations and showing the contributions of the individual stages (Cowley et al., 1991).

Allowing for the increase of $V_{0}$ down the chain thus gives

$$
V_{0} \approx 31-0.15 E \text {. }
$$

This is plotted in Fig. 25 and has an energy dependence similar to the empirical values.

A rather better fit to the overall energy dependence can be obtained using the expression found by Johnson et al. (1987) for neutrons on lead

$$
V=46.4 \exp \left[-0.31\left(E-E_{\mathrm{F}}\right) / 46.4\right] \text {. }
$$

Taking $E=-6 \mathrm{MeV}$, normalising and introducing the factor $\frac{3}{4}$ as before gives

$$
V=30.8 \exp (-0.162 E / 30.8) \text {, }
$$

which is also compared with the empirical values in Fig. 25.

More direct evidence of the energy dependence of the effective interaction is provided by the analysis of the $\mathrm{Zr}(\mathrm{p}$, d) $\mathrm{Zr}$ reaction at energies from 20 to $120 \mathrm{MeV}$ by Kosugi and Kosugi (1983). In order to obtain energy-independent spectroscopic factors they found it necessary to allow the effective interaction to vary with energy. Normalising their result to that of Austin gives

$$
V_{0} \approx 31.5-0.12 E \text {, }
$$

which is very similar to that found above. 


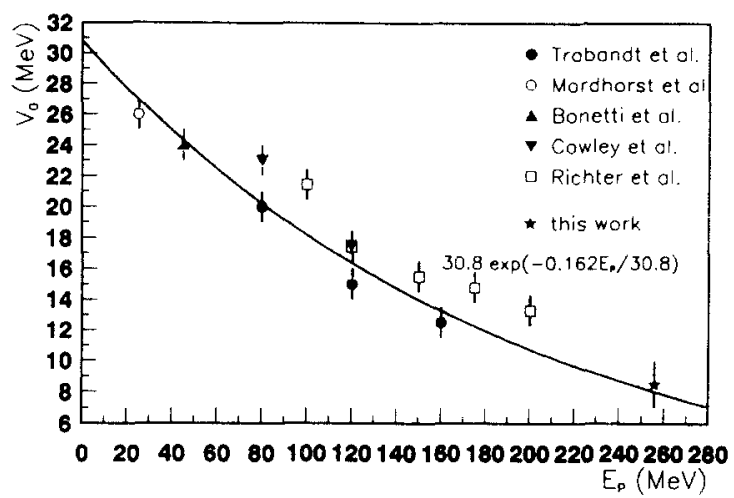

Fig. 25. The effective interaction strength $V$ as a function of incident energy for nucleons with the Yukawa form factor with range $1 \mathrm{fm}$. The use of the delta function effective interaction decreases the value of $V_{0}$ by a factor of about three, as determined by calculations on the same reaction with both types of effective interaction (Bonetti and Colombo, 1983). It is also notable that the same value of $V_{0}$ is obtained from both the multistep compound and multistep direct analyses of reactions to which both processes contribute substantially (Mordhorst et al., 1986). The curve is obtained from the energy dependence of the optical model potential. The references for the data points are given in Table 1 (Stamer et al., 1993).

Table 2

Values of the effective strength $V_{0}$ of the Yukawa potential of range $1 \mathrm{fm}$ for (p, p') interactions (Richter et al.. 1992)

\begin{tabular}{|c|c|c|c|c|c|}
\hline \multirow[t]{2}{*}{ Target } & \multicolumn{5}{|c|}{ Energy } \\
\hline & 100 & 120 & 150 & 175 & 200 \\
\hline${ }^{58} \mathrm{Ni}$ & 23.8 & 23.0 & 21.0 & 20.5 & 18.0 \\
\hline${ }^{100} \mathrm{Mo}$ & 22.0 & 16.7 & 14.0 & 13.5 & 12.5 \\
\hline${ }^{197} \mathrm{Au}$ & 18.5 & 12.5 & 11.5 & 10.3 & 9.4 \\
\hline
\end{tabular}

The values of $V$ obtained by Richter et al. (1992) given in Table 2 show a definite tendency to fall with increasing target atomic mass. This may be further investigated by removing the energy dependence by using (3.4) and thus plotting $V_{0} \exp (-0.00486 E)$ against $A$, as shown in Fig. 26. The data are approximately fitted by the relation

$$
V_{0}=\frac{307}{A^{\frac{1}{2}}} \exp (-0.00486 E)
$$

\subsection{A macroscopic computational model}

An alternative computational approach to MSD reactions was developed by Koning and Akkermans (1993). Instead of averaging over many microscopic DWBA cross-sections for different particle-hole states with the same total angular momentum, macroscopic DWBA cross-sections for each energy bin and total angular momentum are used for the MSD calculation. The calculations have only one free parameter, the deformation parameter $\beta$. Koning $(1992 a, b)$ has 


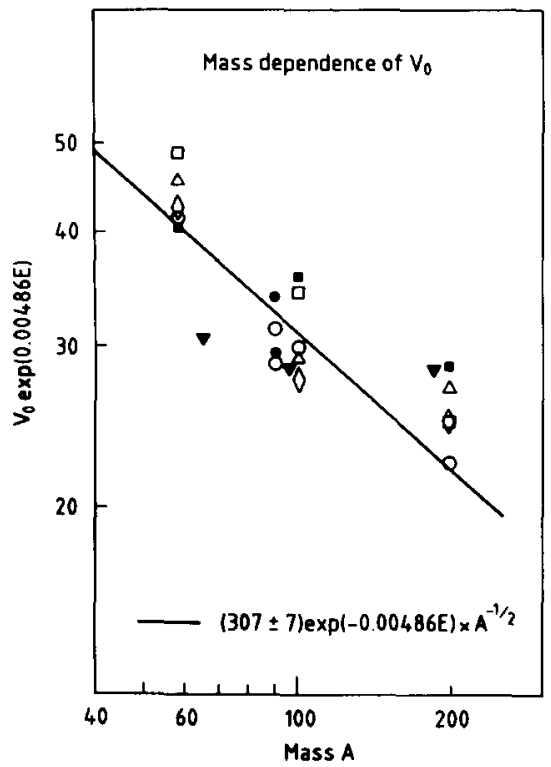

Fig. 26. The effective interaction strength $V_{0}$ as a function of $A$.

obtained a parametrization for $\beta$,

$$
\beta^{2}=\frac{2000}{A^{3} E}
$$

where $A$ is the mass number and $E$ the incident energy. The same energy dependence for $V_{0}$ has been found by Chadwick and Young (1993). In Fig. 27 the results of such an MSD calculation are compared with experimental data and with the angular distribution systematics of Kalbach (1988). At high outgoing energies, both calculations are in excellent agreement with the experimental data. At the lowest outgoing energy, the calculated MSD cross-sections are below the experimental data, although the shape of the angular distribution is still reasonably satisfactory. Such underprediction is to be expected, since the role of other competing processes, such as multiple emission and $P$ - to $Q$-chain transitions become significant at lower outgoing energies.

Calculations were also made using the MSD theories of Tamura et al. (1982) (TUL) and of Nishioka, Weidenmüller and Yoshida $(1989,1990)$ (NWY), and the results are shown in Figs. 28 and 29. Although the FKK, TUL and NWY models are physically very different, they give very similar fits to the experimental cross-sections. Feshbach (1992) suggests that polarisation experiments would distinguish between the FKK and other models, but this is an open question as it has not yet been studied in sufficient detail. However, preliminary calculations (Koning and Akkermans, 1993b) suggest that for the analysing powers as well the calculated differences between the various models are rather small and tend to fall within the experimental error bands. Hence, it appears to be difficult to discriminate between the TUL, NWY and FKK model predictions by comparison with experimental data. However, since the FKK model is computationally the simpler it is preferable for routine calculations. 

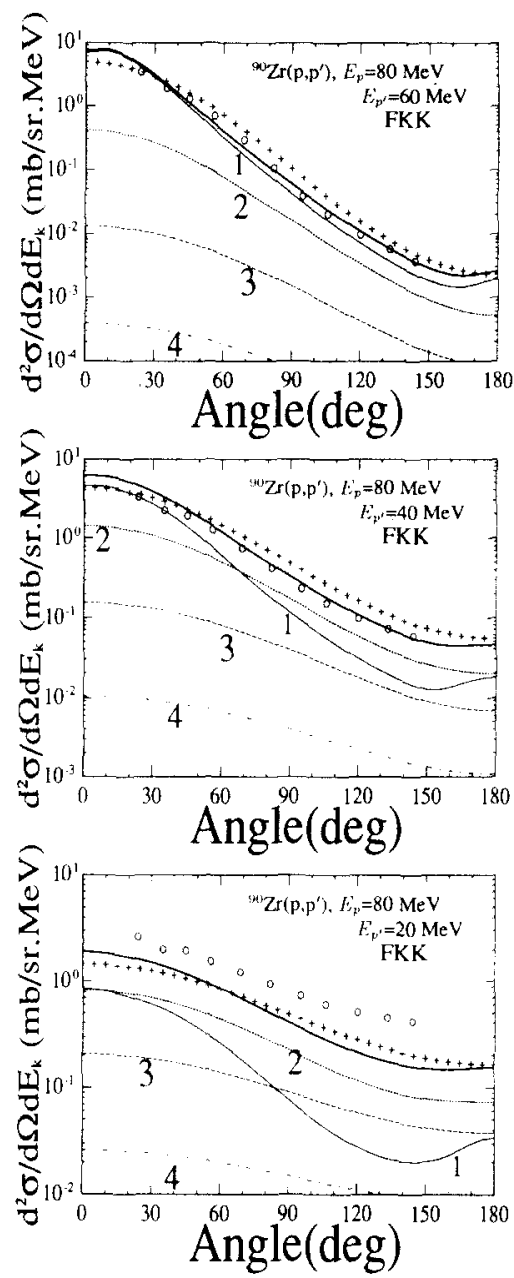

Fig. 27. Angular distributions for the ${ }^{90} \mathrm{Zr}\left(\mathrm{p}, \mathrm{p}^{\prime}\right)$ reaction at $80 \mathrm{MeV}$ (Cowley et al., 1991) for three outgoing energies compared with FKK MSD calculations. The numbered curves give the contributions of the first four steps and the full curve the total MSD cross-section. The circles show the experimental data and the pluses the systematics of Kalbach (Koning and Akkermans, 1991).

\subsection{Transitions from the $P$ - to the $Q$-chain}

The calculations described in the previous sections were made assuming that the reactions proceed either by the multistep direct $P$-chain or by the multistep compound $Q$-chain processes, and the experimental data are fitted by the former at lower energies and by the latter at higher energies. Some analyses at intermediate energies use an incoherent superposition of the two processes. There is, however, some evidence that this distinction between $P$ - and $Q$-chain processes is too sharp, and that it is possible for transitions to take place from the $P$-chain to the $Q$-chain after the first step. Thus if we consider reactions due to $14 \mathrm{MeV}$ neutrons on ${ }^{93} \mathrm{Nb}$, the total flux into 

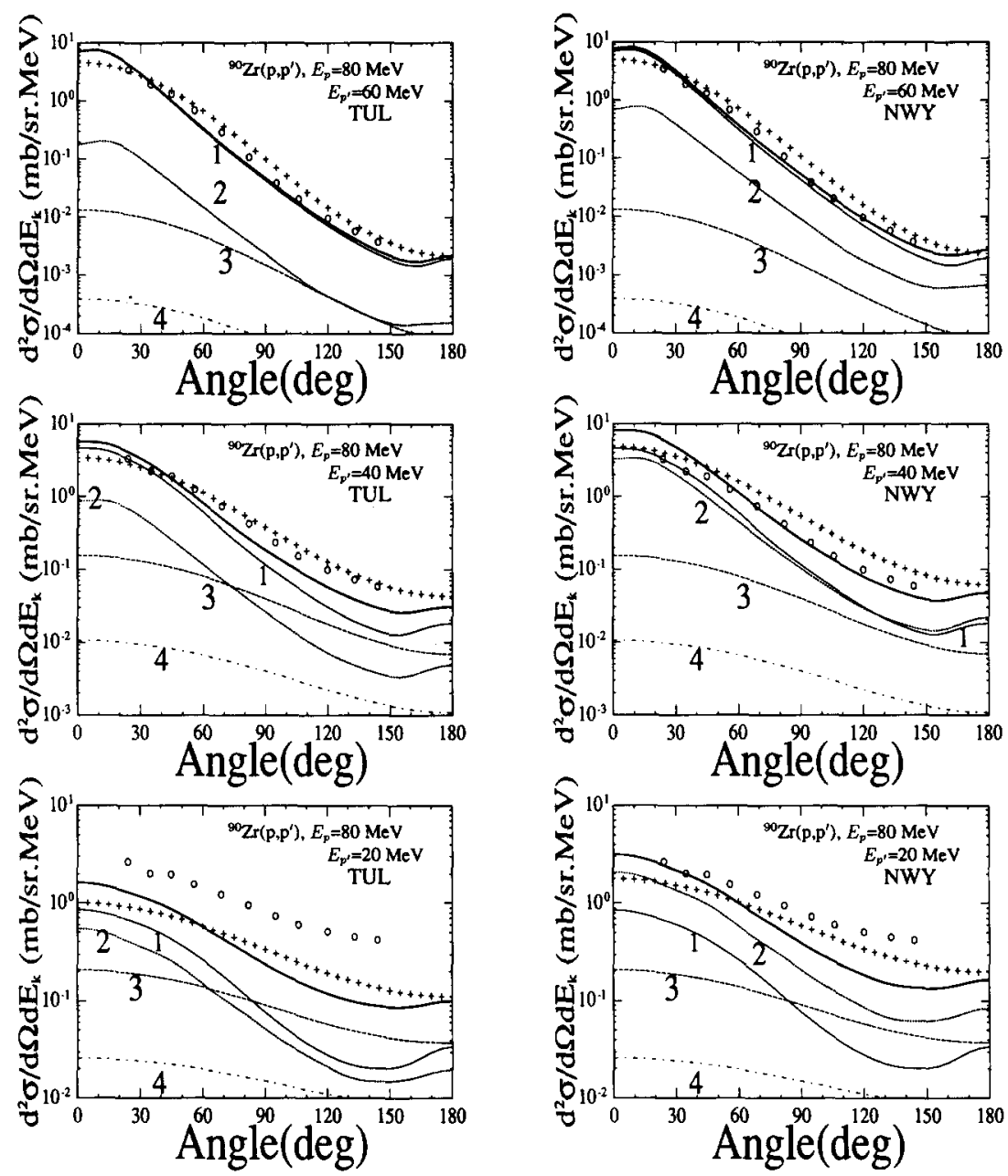

Fig. 28. Angular distributions for the ${ }^{90} \mathrm{Zr}\left(\mathrm{p}, \mathrm{p}^{\prime}\right)$ reaction at $80 \mathrm{MeV}$ (Cowley et al., 1991) for three outgoing energies compared with TUL MSD calculations. The numbered curves give the contributions of the first four steps and the full curve the total MSD cross-section. The circles show the experimental data and the pluses the systematics of Kalbach (Koning and Akkermans, 1991).

Fig. 29. Angular distributions for the ${ }^{90} \mathrm{Zr}\left(\mathrm{p}, \mathrm{p}^{\prime}\right)$ reaction at $80 \mathrm{MeV}$ (Cowley et al., 1991) for three outgoing energies compared with NWY MSD calculations. The numbered curves give the contributions of the first four steps and the full curve the total MSD cross-section. The circles show the experimental data and the pluses the systematics of Kalbach (Koning and Akkermans, 1991).

bound $2 p l h$ states calculated using the value of $V_{0}$ obtained by fitting the double differential cross-section for inelastic scattering is found to be $70 \%$ of the total absorption flux. However, the fraction of compound emission found from the asymmetry of the emitted neutrons is $86 \pm 5 \%$ (Chadwick, 1989). This indicates that about $16 \%$ of the flux enters the $Q$-chain after the first stage, so that the reaction may be represented diagrammatically as in Fig. 30. 


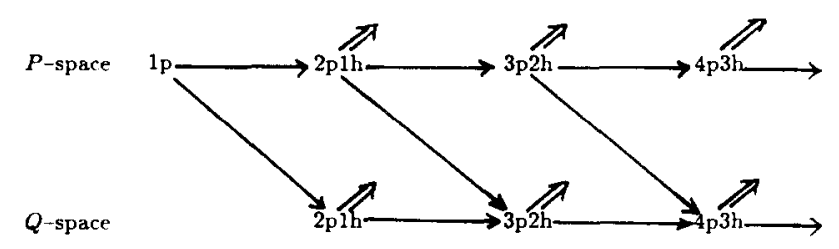

Fig. 30. Multistep reactions with $P$-chain to $Q$-chain transitions (Chadwick. 1989).

The effect of these additional $P$ - to $Q$-chain transitions is to increase the contribution to the emission cross-section from the second and higher stages of the $Q$-chain. At $14 \mathrm{MeV}$ this has very little effect on the summed cross-section, since the contribution from the first stage dominates, but it may become more important at higher energies.

A detailed study of transitions from the $P$ - to the $Q$-chain for neutron inelastic scattering at $20 \mathrm{MeV}$ has been made by Marcinkowski et al. (1991). They define the factor $R$ that gives the proportion of the incident flux going into the MSC process (the $Q$-chain). Thus

$$
\sigma(\mathrm{MSC})=R \sigma_{\mathrm{a}}
$$

so that

$$
\sigma(\mathrm{MSD})=(1-R) \sigma_{\mathrm{a}}
$$

and

$$
\sigma_{\mathrm{a}}=\sigma(\mathrm{MSC})+\sigma(\mathrm{MSD}) .
$$

The factor $R$ can be estimated by assuming that MSD processes contribute only in the forward direction. Then, since the MSC cross-section is symmetric about $90^{\circ}$, the measured asymmetry immediately gives $R$. This is however, on the assumption that the MSD emission is entirely forward-peaked and that after emission of the MSD particle the residual nucleus is in its ground state; if it is not, some compound emission may follow. Another estimate of $R$ can be obtained by calculating the MSD cross-section adjusting $V_{0}$ to fit the forward-peaked part of the cross-section. These two methods gave consistent values of $R=0.8$ for ${ }^{93} \mathrm{Nb}$ and $R=0.7$ for ${ }^{209} \mathrm{Bi}$.

However, with this value of $V_{0}$, the calculated MSC and MSD cross-section exceeded the experimental $\left(\mathrm{n}, \mathrm{n}^{\prime}\right)$ cross-section for ${ }^{209} \mathrm{Bi}$. This can also be expressed by saying that the values of $V_{0}$ found by fitting (a) the forward-peaked components with MSD and (b) the symmetric component with MSC are not consistent.

A consistent account of the data can, however, be obtained by relaxing the assumption of FKK that the MSC and MSD processes remain distinct after the first interaction but that, as already mentioned, transitions can take place from the $P$ - to the $Q$-chain after the first step. Thus the factor $R$ is now replaced by a series of factors $R_{1}^{\prime}, R_{2}^{\prime}, R_{3}^{\prime}$ for each step, with $R_{1}^{\prime}+R_{2}^{\prime}+R_{3}^{\prime}+\cdots=$ $R^{\prime}<R$. It is not possible to determine all these factors from the data, though in principle they could be calculated. A simplified analysis was made (Marcinkowski et al., 1991) using $R^{\prime}<R$ and assuming that $\left(R-R^{\prime}\right) \sigma_{\mathrm{a}}$ goes immediately into the compound nucleus stage. It is then possible to obtain a consistent account of the data with $R^{\prime}=0.6$ for ${ }^{93} \mathrm{Nb}$ and $R^{\prime}=0.4$ for ${ }^{209} \mathrm{Bi}$. 


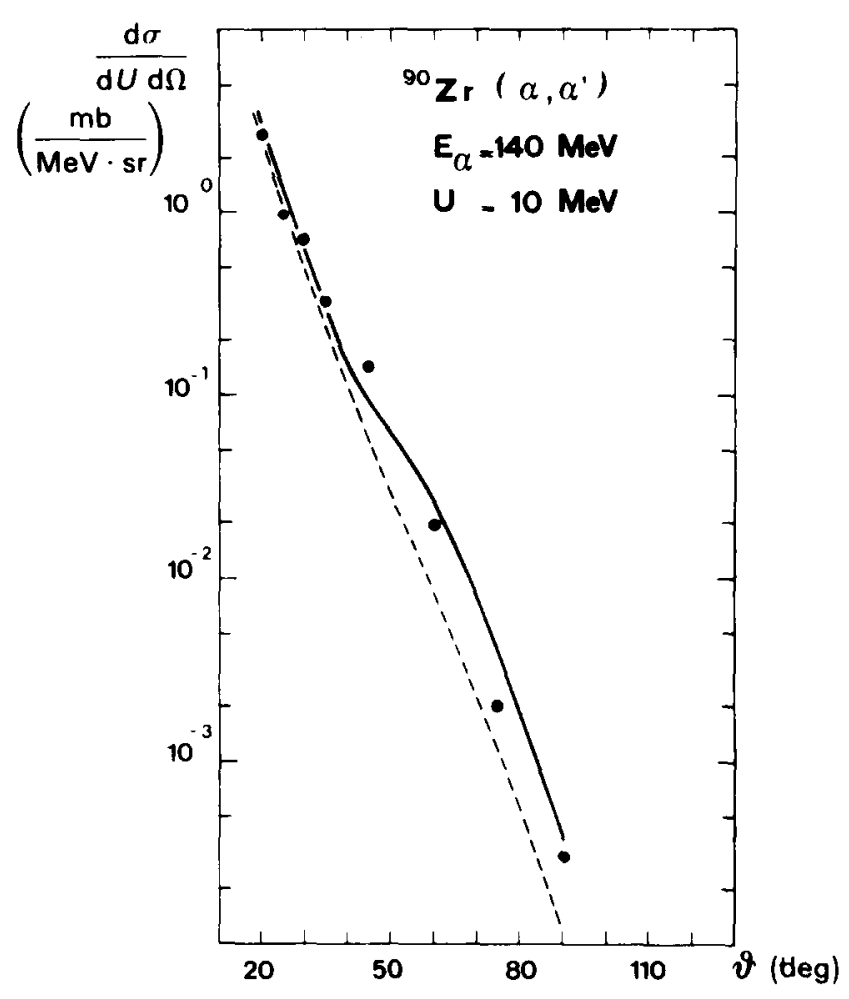

Fig. 31. Double differential cross-section for the inelastic scattering of $140 \mathrm{MeV}$ alpha particles from ${ }^{90} \mathrm{Zr}$ with a final excitation energy of $10 \mathrm{MeV}$. The dashed curve shows the calculated cross-section obtained with the multistep direct theory assuming interactions with alpha particles on the nuclear surface and the full curve is obtained by adding the contribution due to interactions with nucleons (Bonetti et al., 1984).

A model of the processes responsible for the reduction of the first $P$ - to $Q$-chain transition has been proposed by Nishioka et al. (1990). They consider the first interaction to be mainly a sudden direct process that excites only continuum states, followed by the spreading of the remaining $\left(R-R^{\prime}\right) \sigma_{\mathrm{a}}$ flux into the successive stages of the $Q$-chain. These stages make little contribution to the MSC emission because of the rapid increase of the damping width with the number of the stage. The proportions of the first interaction going to the two processes is approximately in the ratio of the number of quasi-bound $2 p 1 h$ doorway states to the total density of accessible particle-hole states, so that $R_{1}^{\prime}=R \sigma_{2 p l h}^{B}\left(E_{p}+B\right) / \sigma_{2 p l h}^{\mathrm{ER} I S O N}\left(E_{p}+B\right)$. At an incident energy of $20 \mathrm{MeV}$ this gives $R^{\prime}=0.16 R$. Thus rather little flux goes to the fourth stage of the $Q$-chain; most of the remainder goes to the second and third stages, so that $R^{\prime}=0.6$.

Chadwick and Young (1993) have studied the importance of $P$ - to $Q$-chain transitions in the inelastic scattering of 14,20 and $25.7 \mathrm{MeV}$ neutrons by ${ }^{93} \mathrm{Nb}$. They assume that the fraction of the incident flux that enters the $Q$-chain is given by the ratio of the bound to the total $2 p 1 h$ phase space. The resulting value of $R$ is about 0.2 , so this gives rather small MSC cross-sections and substantially higher MSD cross-sections. The $P$-chain flux that is not 


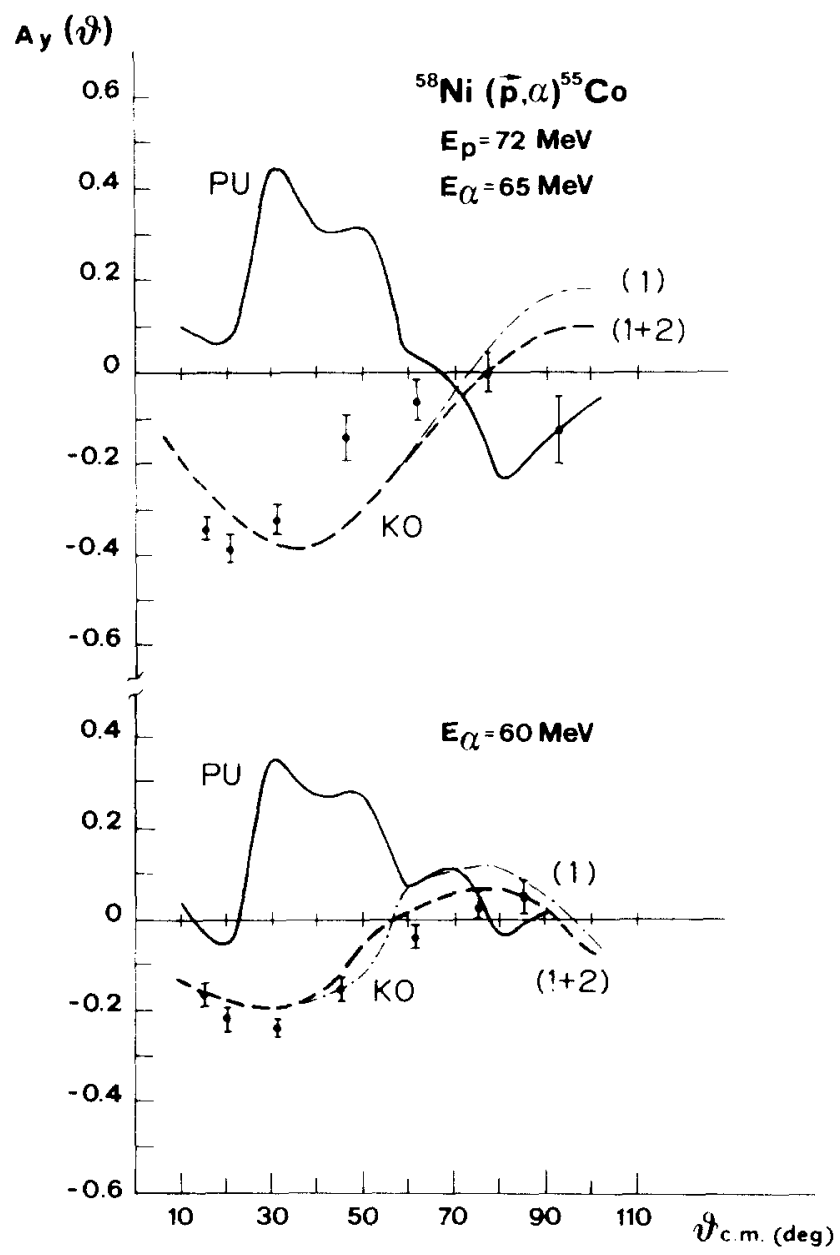

Fig. 32. The analysing power of the ${ }^{58} \mathrm{Ni}\left(\mathrm{p}\right.$, a) ${ }^{55} \mathrm{Co}$ reaction at $72 \mathrm{MeV}$ to the continuum at an excitation energy of $65 \mathrm{MeV}$ compared with multistep direct calculations with the triton pickup (full line) and alpha-particle knockout (dashed line) models. The knockout calculations were made with one step only (1) and with two steps $(1+2)($ Bonetti et al., 1989).

emitted by the MSD process goes to the $Q$-chain and is emitted by the compound nucleus process. They estimate that these $P$ to $Q$ transitions account for about $60 \%$ of the total reaction crosssection at $14 \mathrm{MeV}$.

This estimate is far larger than the figure of $16 \%$ found earlier by Chadwick, but this is due to a change in the definition of a $P$ - to $Q$-chain transition. In his early work the $P$ - to $Q$-chain transitions were those taking place from the first few steps of the $Q$-chain, as shown in Fig. 30 . In the later work, Chadwick and Young include the substantial flux remaining in the $P$-chain after MSD emission, and so obtain a much larger figure. It is thus important to make this distinction when discussing $P$ - to $Q$-chain transitions. 


\subsection{Alpha-particle reactions}

The Feshbach-Kerman-Koonin multistep direct theory has also been used to calculate $\left(\alpha, \alpha^{\prime}\right)$ and $(\mathrm{p}, \alpha)$ cross-sections to continuum states. Bonetti et al. (1984) analysed the ${ }^{90} \mathrm{Zr}\left(\alpha, \alpha^{\prime}\right)$ reaction at $140 \mathrm{MeV}$ to the continuum and found that good fits can be obtained to the angular distributions for residual nucleus excitation energies from 10 to $60 \mathrm{MeV}$ by taking account only of the interaction between the incoming alpha particles and preformed alpha particles on the surface of the target nucleus. The normalisation of the calculations to the data gave values of the alpha clustering probability around 0.1 , in accord with the results of other analyses in that mass region. It was not possible to fit the data with a standard distorted wave Born approximation calculation taking into account only the interaction with the target nucleons. The contributions of these two processes to the angular distribution corresponding to an excitation energy of $10 \mathrm{MeV}$ are shown in Fig. 31.

The $(p, \alpha)$ reaction can take place either by the triton pickup or by the alpha knockout processes, and it is difficult to distinguish between them because they often give the same angular distribution. The analysing power sometimes provides more discrimination, and calculations by Bonetti et al. (1989) using the multistep direct theory showed that at high energies the reaction to the continuum proceeds primarily by the knockout mechanism, as shown in Fig. 32.

\section{Conclusion}

The multistep reaction theory of Feshbach et al. (1980) has now been used to analyse a wide range of experimental data. This work was described for the multistep compound process in the previous review (Bonetti et al., 1991) and for the multistep direct process in the present review, and establishes the theory as a comprehensive and reliable method of calculating the cross-sections of many reactions. It is computationally simpler than the theories of Tamura et al. (1982) and of Nishioka et al. (1989) and since it seems to fit the experimental data equally well, is to be preferred for practical calculations.

During the last few years both the experimental data and the methods of analysis have greatly improved, so that it is now possible to specify the parameters of the theory with some confidence. In general the optical model and level density parameters may be obtained from other work, leaving only the strength $V_{0}$ of the effective nucleon-nucleon interaction to be adjusted to optimise the fit to the data. It is now fairly well established that the same $V_{0}$ suffices for both the multistep compound and the multistep direct reactions, and that it falls exponentially with increasing energy in the same way as the real part of the nucleon optical potential. In the case of proton inelastic scattering there is evidence that $V_{0}$ falls with increasing target mass. It is at present rather less certain that the same $V_{0}$ fits proton and neutron inelastic scattering, $(\mathrm{p}, \mathrm{n})$ and $(\mathrm{n}, \mathrm{p})$ reactions.

There are considerable differences between the estimates of the multistep compound and multistep direct contributions to reactions to which both contribute, and these will have to be resolved before much can be said in a quantitative way about the magnitude of the $P$ - to $Q$-chain transitions. 
At higher energies there is evidence of multiparticle emission, and preliminary calculations indicate (Chadwick, 1993) that this can be understood by an extension of the multistep direct theory.

All this work refers to nucleon reactions, so there is considerable interest in extending the theory to include other emitted particles and incident projectiles. Calculations by Obložinský and Chadwick (1990) have shown that multistep compound gamma emission can account for some of the $(\mathrm{n}, \gamma)$ cross-section, and it remains to be shown that the multistep direct process can account for the remainder. The cross-sections for the emission of alpha particles, deuterons, tritons and other composite particles, together with the whole field of reactions initiated by such particles, constitutes an extensive field for future investigation.

\section{Acknowledgements}

We thank Professor H. Feshbach, Professor M. Kawai and Professor M.S. Hussein for valuable discussions during the preparation of this review.

\section{Appendix by M.B. Chadwick}

The $Y$ functions in multistep compound processes

In this appendix we give expressions for the accessible phase spaces ( $Y$ functions) for transitions in the multistep compound process. While these functions are not of direct relevance to multistep direct processes, there exists a certain amount of confusion about them in the literature, due to either errors in derivations or in the typesetting of the formulae. The $Y$-functions are a critical component to any multistep compound calculation and thus we think it useful to collect together the various expressions, and point out where errors exist in other works.

All FKK analyses to date have used equidistant single particle levels to determine particle--hole state densities (also known as "partial level densities"). The well-known formula of Williams (1971) provides the basis for determining the state densities, but it must be restricted so that only bound single-particle states are included in accordance with the FKK assumption that the MSC chain includes only bound configurations. Of course a restriction on the hole excitations also exists - that they cannot have energies exceeding the Fermi energy - though in practice this restriction can be ignored since it only influences the state densities for incident energies greater than the sum of the Fermi energy and the binding energy, where MSC processes are negligible. Bearing this in mind, the following points can be made about $Y$ functions in the literature.

1. The $Y$ functions were first presented by FKK. These were based on equidistant single-particle states, but did not include the restriction that only bound configurations be included. Also, as was first pointed out by Herman et al. (1984) the $\Delta n=+2$ (increase in the number of excitons by two) process due to particle scattering should not be included because of the bound state restriction.

2. In 1985, Stankiewicz et al. (1985) derived $Y$ functions that satisfy the bound-state-only requirement and that do not include the forbidden particle-scattering $\Delta n=+2$ transition. These are exactly the functions needed for multistep compound calculations. However, their Eq. (17) has an error in it, as pointed out by Obložinský (1986). 
3. In 1986 Obložinský developed expressions for $Y$ functions in an equidistant model in which both restrictions to particle and hole excitations are included. These are, therefore, the most general expressions for $Y$ functions, even though in practice the hole-depth limitations are not important, as mentioned above. Obložinsky's treatment had the added advantage that the notation he introduced considerably simplified the formulae. Expressions for the $Y$ functions in which only particle restrictions are included can be easily obtained from his expressions by taking the limit to infinity of the Fermi energy. Some errors were noticed in this paper by K. Sato, G.M. Field and M.B. Chadwick: Eqs. (16), (18) and (20) have mistakes in them, and Fig. 3 has an error in it.

4. The article on multistep compound emission by Bonetti et al. (1991) (which complements the present multistep direct report), has an error in the $Y$ function in Eq. (3.29). See the errata paper (1992).

5. The paper by Obložinský and Chadwick (1990) on multistep gamma ray emission processes contains correct $Y$ functions (with bound-state restrictions) for both nucleon and gamma-ray emission.

Below we present the $Y$ functions that are obtained in an equidistant single-particle model with bound-state restrictions. See Figs. 3.2 and 3.3 of Bonetti et al. (1991) for the notation. The three emission $Y$ functions are given by (Obložinský and Chadwick, 1990).

$$
\begin{aligned}
Y_{n}^{\dagger n}(U)= & \frac{g^{3}}{\omega^{B}(p, h, E)}\left\{\frac { \Theta ( U - E + 2 B ) } { 2 ( n - 2 ) } \left[(U-E+2 B) \omega^{B}\left(p-2, h, U^{n-2}\right),\right.\right. \\
& \left.+\frac{\omega^{B}\left(p-2, h,[E-2 B]^{n-1}\right)}{n-1}-\frac{\omega^{B}\left(p-2, h, U^{n-1}\right)}{n-1}\right] \\
& \left.+B \frac{\omega^{B}\left(p-1, h-1, U^{n-2}\right)}{n-2}\right\} \\
Y_{n}^{\dagger n+2}(U)= & \frac{g^{3}}{2 \omega^{B}(p, h, E)} \frac{\omega^{B}\left(p, h-1, U^{n}\right)}{n(n-1)}, \\
Y_{n}^{\dagger n-2}(U)= & \frac{\omega^{B}(p-2, h-1, U)}{\omega^{B}(p, h, E)} \omega^{B}(2,1, E-U)
\end{aligned}
$$

for a residual nucleus energy of $U$. The damping $Y$ function is given by

$$
\begin{aligned}
Y_{n}^{\downarrow}= & \frac{g^{4}}{2 \omega^{B}(p, h, E)}\left\{\frac{\omega^{B}\left(p, h-1, E^{n+1}\right)-\omega^{B}\left[p, h-1,(E-B)^{n+1}\right]}{(n-1) n(n+1)}\right. \\
& +\frac{\omega^{B}\left(p-1, h, E^{n+1}\right)-\omega^{B}\left[p-1, h,(E-B)^{n+1}\right]}{(n-1) n(n+1)}-B \frac{\omega^{B}\left[p-1, h,(E-B)^{n}\right]}{(n-1) n} \\
& \left.-B^{2} \frac{\omega^{B}\left[p-1, h,(E-B)^{n-1}\right]}{2(n-1)}\right\} .
\end{aligned}
$$

In the above expressions the density of $p$-particle $h$-hole states is taken from an equidistant single-particle model, with the particles restricted to energies below the binding energy $B$ 
(Stankiewicz et al., 1985; Obložinský, 1986).

$$
\omega^{B}(p, h, E)=\frac{g^{n}}{p ! h !(n-1) !} \sum_{i=0}^{p}(-1)^{i}\left(\begin{array}{l}
p \\
i
\end{array}\right)\left(E-A-A_{p h}-\mathrm{i} B\right)^{n-1} \Theta\left(E-A-A_{p h}-\mathrm{i} B\right),
$$

where $g$ is the single-particle level density, $\Delta$ the pairing energy, and $A_{p h}$ is the Pauli-blocking function. The theta-function is unity if its argument is positive, and zero otherwise. In the above equations we also use the notation of Obložinský (1986)

$$
\omega^{B}\left(p, h, E^{n+H}\right)=\frac{g^{n}}{p ! h !(n-1) !} \sum_{i=0}^{p}(-1)^{i}\left(\begin{array}{l}
p \\
i
\end{array}\right)\left(E-\Delta-A_{p h}-\mathrm{i} B\right)^{n+H} \Theta\left(E-\Delta-A_{p h}-\mathrm{i} B\right) .
$$

which should be used when the exponent $n+H \neq p+h-1$. This notation simplifies the analytic expressions for the $Y$ functions which involve integrations of Eq. (A5).

\section{References}

Akkermans, J.M. and A.J. Koning (1990) Phys. Lett. 234 B, 417.

Akkermans, J.M., H. Gruppelaar and G. Reffo (1980) Phys. Rev. C 22, 73.

Arthur E.D. (1988) in: Proc. Workshop on Applied Nuclear Theory and Nuclear Model Calculations for Nuclear Technology Applications, Trieste, p. 676.

Austern, N. (1970) Direct Nuclear Reaction Theories (Wiley-Interscience) Ch. 4.

Austern, N. and C.M. Vincent (1974) Phys. Rev. C 10, 2623.

Austin, S.M. (1980) The (p,n) Reaction and the Nucleon-Nucleon Force (Plenum, New York).

Avaldi, L., R. Bonetti and L. Colli-Milazzo (1980) Phys. Lett. B 94, 463.

Běták, E. and J. Dobeš (1976) Z. Phys. A 279, 319.

Blann, M. (1975) Ann. Rev. Nucl. Sci. 25, 123.

Blann, M., W. Scobel and E. Plechety (1984) Phys. Rev. C 30, 1493.

Bonetti, R., M. Camnasio, L. Colli-Milazzo and P.E. Hodgson, 1981, Phys. Rev. C 24, 71.

Bonetti, R., L. Colli-Milazzo, I. Doda and P.E. Hodgson (1982) Phys. Rev. C 26, 2417.

Bonetti, R., L. Colli-Milazzo, A. De Rosa, G. Inglima, E. Perillo, M. Sandoli and F. Shahin (1980) Phys. Rev. C. $21,816$.

Bonetti, R., L. Colli-Milazzo, M. Melanotte, A. De Rosa, G. Inglima, E. Perillo, M. Sandoli, V. Russo, N. Saunier and F. Shahin (1982) Phys. Rev. C 25, 717.

Bonetti, R., L. Colli-Milazzo and M. Melanotte (1983) Phys Rev. C 27, 1003.

Bonetti, R. and L. Colli-Milazzo (1984) Proc. Workshop on Coincident Particle Emission from Continuum States in Nuclei, Bad Honnef 4-7 June, eds., H. Machner and P. Jahn (World Scientific, Singapore) 174.

Bonetti, R. and L. Colombo (1983) Phys. Rev. C 28, 980.

Bonetti, R., L. Colombo and K.-I. Kubo (1984) Nucl. Phys. A 420, 109.

Bonetti, R., F. Crespi and K.-I. Kubo (1989) Nucl. Phys. A 499, 381.

Bonetti, R., M.B. Chadwick, P.E. Hodgson, B.V. Carlson and M.S. Hussein (1991) Phys. Rep. 202, 171; Erratum (1992), $211,376$.

Cassing, W. and M. Stingl (1982) Phys. Rev. C 26, 22.

Chadwick, M.B. (1989) Thesis, Oxford; (1993) Paper presented at Rila Conference; (1994) Phys. Rev. C, in press.

Chadwick, M.B. and P.G. Young (1992) in: Symp. on Nuclear Data Evaluation Methodology, Brookhaven National Laboratory, Upton, NY, October 12-16 1992; (1993) Phys. Rev. C 47, 2255.

Costa, C., J.M. Akkermans and H. Gruppelaar (1983) Phys. Rev. C 28, 587. 
Cowley, A.A., A. van Kent, J.J. Lawrie, S.V. Förtsch, D.M. Whittal, J.V. Pilcher, F.D. Smit, W.A. Richter, R. Lindsay, I.J. van Heerden, R. Bonetti and P.E. Hodgson (1991) Phys. Rev. C 43, 678.

Demetriou, P., P. Kanjanarat and P.E. Hodgson (1993) J. Phys. 19, L193.

Feshbach, H. (1962) Ann. Phys. (NY) 19, 287.

Feshbach, H., A.K. Kerman and R.H. Lemmer (1967) Ann. Phys. (NY) 41, 230.

Feshbach, H. (1973) in: Proc. Int. Conf, on Nuclear Physics, Munich, eds., J.J. de Boer and H.J. Mang (North-Holland, Amsterdam) Vol. II, p. 631.

Feshbach, H. (1977) in: Proc. Int. Conf. on Nuclear Reaction Mechanisms, Varenna (CLUED) p. 1.

Feshbach, H., A. Kerman and S. Koonin (1980) Ann. Phys. 125, 429.

Feshbach, H. (1984) in: Proc. Winter College on Fundamental Nuclear Physics, Trieste, eds., K. Dietrich, M. di Toro and H.J. Mang (World Scientific, Singapore) Vol. 3, p. 1605.

Feshbach, H. (1985a) Ann. Phys. 159, 150; (1985b) 164, 398.

Feshbach, H. (1984) in: Proc. Int. School of Physics. Course XCI, eds., A. Molinari and R.A. Ricci, Varenna (NorthHolland, Amsterdam) p. 116.

Feshbach, H. (1986b) Phys. Lett. B 168, 318.

Feshbach, H. (1992) in: Proc. Workshop on Multistep Direct Reactions, Faure; ed., R.H. Lemmer (World Scientific, Singapore) pp. 1, 9, 223.

Feshbach, H. (1993) private communication to A.J. Koning, J.M. Akkermans and P.E. Hodgson.

Gadioli, E. and E. Gadioli Erba (1981) Nuclear Theory for Applications, ICTP Report IAEA-SMR-68/1.

Gadioli, E. and P.E. Hodgson (1992) Pre-equilibrium Nuclear Reactions (Clarendon Press, Oxford).

Gal, A., G. Toker and Y. Alexander (1981) Ann. Phys. (NY) 137, 341.

Galonsky, A., Personal communication to R. Bonetti et al. 1981.

Griffin, J.J. (1966) Phys. Rev. Lett. 17, 478.

Gruppelaar, H., P. Nagel and P.E. Hodgson (1981) Riv. Nuovo. Cim. 9, 1.

Gruppelaar, H. and P. Nagel (1985) Newsletter of the NEA Data Bank NEANDC-1, 204 "U".

Gruppelaar, H., P. Nagel and P.E. Hodgson (1986) Nuovo Cim. 9.

Harp, G.D., J.M. Miller and B.J. Berne (1968) Phys. Rev. 165, 1166.

Herman, M., A. Marcinkowski and K. Stankiewicz (1984) Nucl. Phys. A430, 69.

Hodgson, P.E. (1992) in: Proc. Workshop on Multistep Direct Reactions held at the National Accelerator Centre, Faure, South Africa, ed., R.H. Lemmer (World Scientific, Singapore) p. 9.

Holler, Y., A. Kaminsky, R. Langkau, W. Scobel, M. Trabandt and R. Bonetti (1985) Nucl. Phys. A 442, 79.

Hussein, M.S. and R. Bonetti (1982) Phys. Lett. B 112, 189.

Hussein, M.S. (1987) Ann. Phys. (NY) 175, 197; (1988) Ann. Acad. Bras. Ci. 60, 19.

Iwamoto, A. and K. Harada (1984) Nucl. Phys. A 419, 472.

Jacob, G. and T.A.J. Maris (1973) Rev. Mod. Phys. 45, 6.

Joffily, S. (1973) Nuc. Phys. A 215, 301.

Johnson, C.H., D.J. Horen and C. Mahaux (1987) Phys. Rev. C 36, 2252.

Kalbach, C. (1988) Phys. Rev. C 37, 2350.

Kalka, H., M. Torjman and D. Seeliger (1988) in: Proc. Specialists' Meeting on Pre-Equilibrium Nuclear Reactions, Semmering, Austria, ed., B. Strohmaier, NEANDC-245 "U", p. 305.

Kawai, M., private communication to H. Feshbach and A. Kerman.

Koning, A.J. (1992a) in: Symp. on Nuclear Data Evaluation Methodology, Brookhaven National Laboratory, Upton, New York, October 12-16.

Koning, A.J. (1992b) in: Proc. Workshop on Multistep Direct Reactions held at the National Accelerator Centre, Faure, South Africa, ed., R.H. Lemmer (World Scientific, Singapore).

Koning, A.J. and J.M. Akkermans (1991) Ann. Phys. 208, 216.

Koning, A.J. and J.M. Akkermans (1992) in: Proc. OECD Meeting Nuclear Level Densities, held at Bologna, Italy, 1989, eds., G. Reffo, M. Herman and G. Maino (World Scientific, Singapore) p. 157.

Koning, A.J. and J.M. Akkermans (1993a) Phys. Rev. C 47, 724; (1993b) private communication.

Kosugi, S. and T. Kosugi (1983) Phys. Lett. 127 B, 389.

Kumabe, I., M. Haruta, M. Hyakutake and M. Matoba (1984) Phys. Lett. B 104, 272. 
Lenske, H., S. Landowne, H.H. Wolter, T. Tamura and T. Udagawa (1983) Phys. Lett. B 123, 333.

Luo, Y.L. and M. Kawai (1991) Phys. Rev. C 43, 2367.

Mantzouranis, G., H.A. Weidenmüller and D. Agassi (1976) Z. Phys. A 276, 145.

Marcinkowski, A., R.W. Finlay, G. Sanders-Pehrson, C.E. Briant, J.E. O’Donnell and K. Stankiewicz (1983a) Nucl. Phys. A 202,220 .

Marcinkowski, A., R.W. Finlay, G. Randers-Pehrson, C.E. Brient, R. Kurup, S. Mellema. A. Meigoni and R. Tailor (1983b) Nucl. Sci. Eng. 83, 13.

Marcinkowski, A., R.W. Finlay, J. Rapaport, P.E. Hodgson and M.B. Chadwick (1989) Nucl. Phys. A 501. 1.

Marcinkowski, A., J. Rapaport, R. Finlay, X. Aslanoglou and D. Kielan (1991) Nucl. Phys. A $530,75$.

Mordhorst, E., M. Trabandt, A. Kaminsky, H. Krause, W. Scobel, R. Bonetti and F. Crespi (1986) Phys. Rev. C 34. 103.

Nishioka, H., H.A. Weidenmüller and S. Yoshida (1988) Ann. Phys. (NY) 183. 166.

Nishioka, H. and H.A. Weidenmüller (1988) Phys. Lett. B 203,1.

Nishioka, H., H.A. Weidenmüller and S. Yoshida (1989) Ann. of Phys. (NY) 193. 195; (1990) Z. Phys. A $336,197$.

Obložinský, P. (1986) Nucl. Phys. A 453, 127.

Obložinsky', P. and M.B. Chadwick (1990) Phys. Rev. C 42, 1652

Richter, W.A., A.A. Cowley, R. Lindsay, J.J. Lawrie, S.V. Förtsch, J.V. Pilcher, R. Bonetti and P.E. Hodgson (1992) Phys. Rev. C 46, 1030.

Richter, W.A., A.A. Cowley, G.C. Hillhouse, J.A. Stander, J.W. Koen, S.W. Steyn, R. Lindsay, R.E. Julies, J.J. Lawrie, J.V. Pilcher and P.E. Hodgson, (1994) Phys. Rev. C 49, 717.

Robson. D. (1973) Phys. Rev. C 7. 1.

Sakai, H., K. Hosono, N. Matsuoka, S. Nagamachi, K. Okada, K. Maeda and H. Shimizu (1980) Nucl. Phys. A 344,41 Satchler, G.R. (1983) Direct Nuclear Reactions (Oxford University Press, New York).

Scobel, W., M. Trabandt, M. Blann, B.A. Pohl, B.R. Remington, R.C. Byrd, C.C. Foster, R. Bonetti, C. Chiesa and S.M. Grimes (1990) Phys. Rev. C 41, 2010.

Stamer, S., W. Scobel, W.B. Amian, R.C. Byrd, R.C. Haight, J.L. Ullmann, R.W. Bauer, M. Blann, B.A. Pohl, J. Bisplinghoff and R. Bonetti (1993) Phys. Rev. C 47, 1647.

Stankiewicz, K., A. Marcinkowski and M. Herman (1985) Nucl. Phys. A 435, 67.

Takahashi, A., M. Gotoh, Y. Sasaki and H. Sugimoto (1992) OKTAVIAN Report A-92-91-1992.

Tamura, T., T. Udagawa, D.H. Feng and K.K. Kan (1977) Phys. Lett. B 66, 109.

Tamura, T. and T. Udagawa (1977a) Phys. Lett. B 71, 273.

Tamura, T., T. Udagawa, D.H. Feng and K-K. Kan (1977b) Phys. Lett. B 66, 109.

Tamura, T. and T. Udagawa (1978) Phys. Lett. B 78, 1989.

Tamura, T., H. Lenske and T. Udagawa (1981) Phys. Rev. C 23, 2769.

Tamura, T., T. Udagawa and H. Lenske (1982) Phys. Rev. C 26, 379.

Trabandt, M., M. Blann, R. Bonetti, R. C. Byrd, C. C. Foster, S. M. Grimes, B.A. Pohl, B.R. Remington and W. Scobel (1988) in: Proc. 5th Int. Conf. on Nuclear Reaction Mechanisms, ed., E. Gadioli. (Varenna) p. 52.

Trabandt, M., W. Scobel, M. Blann, B. Pohl, R.C. Byrd, C.C. Foster and R. Bonetti (1989) Phys. Rev. C $29,452$.

Tsai, S. and G.F. Bertsch (1978) Phys. Lett. B 73, 247.

Udagawa, T., T. Tamura and B.T. Kim (1979) Phys. Lett. B 82, 349.

Udagawa, T. and T. Tamura (1980) Continuum Spectra in Heavy Ion Reactions, eds., T. Tamura, J.B. Natowitz and D.H. Youngblood (Harwood, New York) p. 155.

Udagawa, T., D. Price and T. Tamura (1982) Phys. Lett. B 116, 311.

Udagawa, T., K.S. Low and T. Tamura (1983) Phys. Rev. C 28, 1033.

Williams, F.C. (1971) Nucl. Phys. A 166, 231.

Young, P.G., E.D. Arthur and M.B. Chadwick (1992) Los Alamos National Laboratory Report LA-12343-MS. 